Aus der Abteilung Kardiologie und Pneumologie

(Prof. Dr. med. G. Hasenfuß)

im Zentrum Innere Medizin

der Medizinischen Fakultät der Universität Göttingen

\title{
Einfluss milder therapeutischer Hypothermie auf die respiratorische und kardiale Funktion nach primär erfolgreicher kardiopulmonaler Reanimation
}

\author{
INAUGURAL-DISSERTATION \\ zur Erlangung des Doktorgrades \\ der Medizinischen Fakultät \\ der Georg-August-Universität zu Göttingen
}

\author{
vorgelegt von \\ Anja Teresa Pax \\ aus \\ Köln
}

Göttingen 2013 
Dekan:

1. Berichterstatter:

2. Berichterstatter:

3. Berichterstatter:
Prof. Dr. rer. nat. H. K. Kroemer

Priv.-Doz. Dr. med. C. Jacobshagen

Prof. Dr. med. Bräuer

Priv.-Doz. Dr. med. Sigler

Tag der mündlichen Prüfung: 07.11.2013 


\section{Inhaltsverzeichnis}

II.a Abbildungsverzeichnis ……………………………………………..... VIII

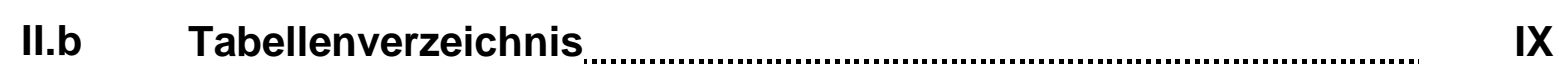

\section{Einleitung}

1.1 Herzkreislaufstillstand und kardiopulmonale Reanimation

- Relevanz und Häufigkeit

1.2 Postreanimationssyndrom .............................................................. 2

$1.3 \quad$ Milde therapeutische Hypothermie ………………....................... 5

1.3.1 Einsatz nach kardiopulmonaler Reanimation ..................................... 5



1.3.3 Kühlungsmethoden ....................................................................... 10

1.3.4 Weitere Therapiestrategien ………………………………………….... 11

$1.4 \quad$ Zielsetzung der Arbeit ............................................................................ 13

$2 \quad$ Methoden

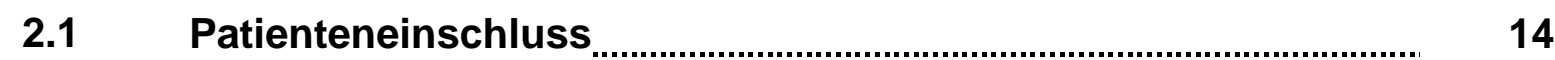

2.1.1 Datenerhebung für die respiratorische Funktion ................................... 16

2.1.2 Datenerhebung für die kardiale Funktion und Hämodynamik ............. 16

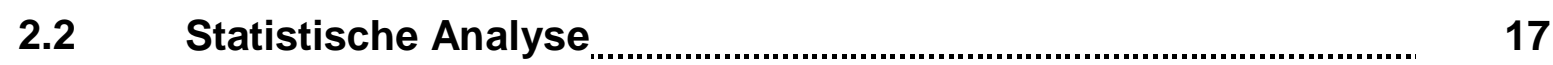

$3 \quad$ Ergebnisse

$\begin{array}{lll}3.1 & \text { Einfluss milder Hypothermie auf die respiratorische Funktion... } & 18\end{array}$

3.1.1 Basisdaten ....................................................................................... 18

3.1.2 Parameter zur milden Hypothermie _...................................................... 20

3.1.3 Parameter der respiratorischen Funktion ................................................ 22 
3.2 Einfluss milder Hypothermie auf die Herz-Kreislauf-Funktion .... 26

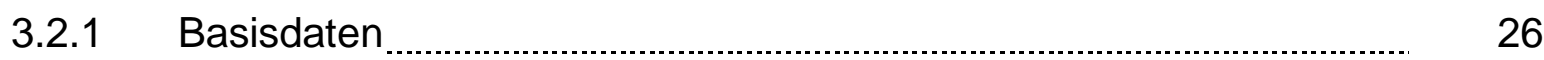

3.2.2 Parameter zur milden Hypothermie _....................................................... 28

3.2.3 Parameter der Herz-Kreislauf-Funktion ................................................. 30

3.2.3.1 Einfluss auf die Katecholamin-Dosierung.............................................. $\quad 30$

3.2.3.2 Einfluss auf Blutdruck und Herzfrequenz ................................................ $\quad 35$

3.2.3.3 Einfluss des pH-Wertes auf die Katecholamin-Dosierung _............... 37

$4 \quad$ Diskussion

$\begin{array}{lll}\text { 4.1 Einfluss milder Hypothermie auf die respiratorische Funktion .. } & 39\end{array}$

4.2 Kühlungsgeschwindigkeit unter Gabe kalter Infusionen .............. 42

4.3 Einfluss milder Hypothermie auf die Herz-Kreislauf-Funktion ..... 43

4.3.1 Einfluss auf die Adrenalin-Dosierung _............................................... 44

4.3.2 Einfluss auf die Noradrenalin-Dosierung ................................................ 46

4.3.3 Einfluss auf Blutdruck und Herzfrequenz _........................................ 4

4.3.4 Einfluss des pH-Wertes auf die Katecholamin-Dosierung ................... 49

4.4 Limitationen der Datengewinnung und -interpretation ................. 49



$5 \quad$ Zusammenfassung.

52

$6 \quad$ Anhang

6.1 Statistische Daten ..................................................................... 54

6.2 Abbildungen und Daten nach Aufteilung $\mathrm{PCl}=0$ und $\mathrm{PCl}=1 \ldots . .57$

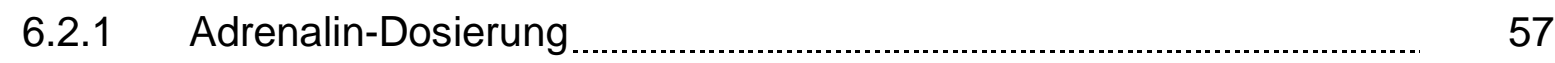

6.2.2 Dobutamin-Dosierung........................................................................... 58

6.2.3 Mittlerer arterieller Blutdruck ................................................................ 59

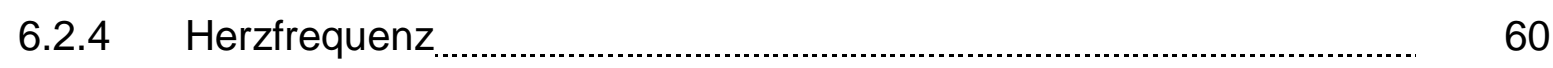

$6.2 .5 \mathrm{pH}-$ Wert $\ldots 1$

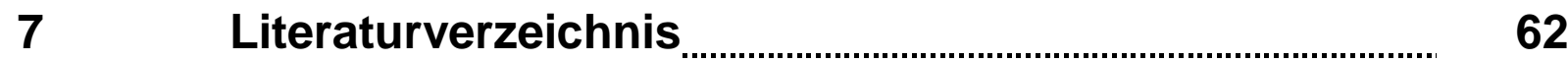




\section{Abkürzungsverzeichnis}

$\%$

Abb.

ACS

Ant.

AP

ARDS

ATP

$B G A$

bspw.

bzw.

${ }^{\circ} \mathrm{C}$

$\mathrm{Ca}^{2+}$

$\mathrm{Cl}$

CPR

CT

Echo

$\mathrm{EF}$

EKG

ER

ERC

etc.

evtl.

$\mathrm{FiO}_{2}$

$\mathrm{h}$

HACA

$\mathrm{HF}$

HKL

HKS

$\mathrm{HR}$

$\mathrm{HZV}$
Prozentsatz

Abbildung

akutes Koronarsyndrom

Anteil mit Katecholaminen behandelter Patienten

Aktionspotenzial

akutes Atemnotsyndrom

Adenosintriphosphat

Blutgasanalyse

beispielsweise

beziehungsweise

Grad Celsius

Kalzium

Herzindex

kardiopulmonale Reanimation

Computertomographie

Echokardiographie

Ejektionsfraktion

Elektrokardiogramm

Notaufnahme

European Resuscitation Council

et cetera

eventuell

inspiratorische Sauerstoffkonzentration

Stunde

Hypothermia After Cardiac Arrest Study Group

Herzfrequenz

Herzkatheter-Labor

Herz-Kreislauf-Stillstand

Herzrhythmus

Herzzeitvolumen 


$\begin{array}{ll}\text { IABP } & \text { intraaortale Ballonpumpe } \\ \text { IHCA } & \text { Herzkreislaufstillstand innerhalb des Krankenhauses } \\ \text { IL } & \text { Interleukin } \\ \text { ILCOR } & \text { International Liaison Committee on Resuscitation } \\ \text { IQR } & \text { Interquartilsabstand } \\ \text { ITS } & \text { Intensivtherapiestation } \\ \text { KF } & \text { Kammerflimmern } \\ \text { kg } & \text { Kilogramm } \\ \text { KG } & \text { Körpergewicht } \\ \text { KKT } & \text { Körperkerntemperatur } \\ \text { LVEDP } & \text { linksventrikulärer enddiastolischer Druck } \\ \text { LVEF } & \text { linksventrikuläre Ejektionsfraktion } \\ \text { LVESP } & \text { linksventrikulärer endsystolischer Druck } \\ \text { LV-Funktion } & \text { linksventrikuläre Funktion } \\ \text { MAP } & \text { mittlerer arterieller Blutdruck } \\ \text { MAX } & \text { Maximum } \\ \text { mbar } & \text { Millibar } \\ \text { Hg } & \text { Mikrogramm } \\ \text { min } & \text { Minute } \\ \text { MIN } & \text { Minimum } \\ \text { ml } & \text { Milliliter } \\ \text { mmHg } & \text { Millimeter-Quecksilbersäule } \\ \text { MTH } & \text { milde therapeutische Hypothermie } \\ \text { MW } & \text { Mittelwert } \\ \mathrm{n} & \text { Gesamtzahl } \\ \mathrm{NA} & \text { Noradrenalin } \\ \mathrm{NaCl} & \text { Natriumchlorid } \\ \mathrm{NRCPR} & \text { National Registry of Cardiopulmonary Resuscitation } \\ \mathrm{n} . \mathrm{s} . & \text { nicht signifikant } \\ \mathrm{NSE} & \text { neuronenspezifische Enolase } \\ \mathrm{O}_{2} & \text { Sauerstoff } \\ \mathrm{OHCA} & \text { Herzkreislaufstillstand außerhalb des Krankenhauses } \\ \mathrm{P} & \\ \mathrm{PaCO}{ }_{2} & \end{array}$




$\begin{array}{ll}\text { PA-Katheter } & \text { Pulmonalarterien-Katheter } \\ \mathrm{PaO}_{2} & \text { arterieller Sauerstoff-Partialdruck } \\ \mathrm{PCAS} & \text { Postreanimationssyndrom } \\ \mathrm{PCI} & \text { perkutane koronare Intervention } \\ \mathrm{PEA} & \text { pulslose elektrische Aktivität } \\ \mathrm{PEEP} & \text { positiver endexspiratorischer Druck } \\ \mathrm{Pmax} & \text { Beatmungsspitzendruck } \\ \text { 25\%-Q } & \text { 25\%-Quantil } \\ 75 \%-\mathrm{Q} & \text { 75\%-Quantil } \\ \mathrm{r}^{2} & \text { Bestimmtheitsmaß } \\ \mathrm{ROSC} & \text { Wiederherstellung eines Spontankreislaufs } \\ \text { SaO } & \text { arterielle Sauerstoffsättigung } \\ \text { SD } & \text { Standardabweichung } \\ \text { SEM } & \text { Standardfehler des arithmetischen Mittels } \\ \text { SIRS } & \text { systemisches inflammatorisches Response-Syndrom } \\ \text { Spw. } & \text { Spannweite } \\ \text { SR } & \text { sarkoplasmatisches Retikulum } \\ \text { sTNFRII } & \text { löslicher TNF-Rezeptor II } \\ \text { SV } & \text { Schlagvolumen } \\ \text { Tab. } & \text { Tabelle } \\ \text { TNF } & \text { Tumornekrosefaktor } \\ \text { TPR } & \text { totaler peripherer Widerstand } \\ \text { UMG } & \text { Universitätsmedizin Göttingen } \\ \text { V.a. } & \text { vor allem } \\ \text { ZVK } & \text { zentraler Venenkatheter } \\ \end{array}$




\section{Il Abbildungs- und Tabellenverzeichnis}

\section{II.a Abbildungsverzeichnis}

Abb. 3.1 Boxplots zum Verlauf der respiratorischen Parameter

Abb. 3.2 Scatterplot zur Visualisierung der Korrelation zwischen Horovitz-Quotient $\left(\mathrm{PaO}_{2} / \mathrm{FiO}_{2}\right)$ bei $34^{\circ} \mathrm{C}$ und der Menge des bis dahin infundierten kalten Volumens

Abb. 3.3 Scatterplot zur Visualisierung der Korrelation zwischen der Änderungsrate des Horovitz-Quotienten $\left(\mathrm{PaO}_{2} / \mathrm{FiO}_{2}\right)$ bei $34^{\circ} \mathrm{C}$ und der Menge des bis dahin infundierten kalten Volumens

Abb. 3.4 Scatterplot zur Visualisierung der Korrelation zwischen der Änderungsrate des Horovitz-Quotienten $\left(\mathrm{PaO}_{2} / \mathrm{FiO}_{2}\right)$ bei $34^{\circ} \mathrm{C}$ und der Ejektionsfraktion..

Abb. 3.5 Boxplots zum Verlauf der Noradrenalin-Dosierung.

Aufspaltung in $\mathrm{PCl}=0$ und $\mathrm{PCl}=1$ 32

Abb. 3.6 Boxplots zum Verlauf der Adrenalin-Dosierung. 33

Abb. 3.7 Boxplots zum Verlauf der Dobutamin-Dosierung. 34

Abb. 3.8 Boxplots zum Verlauf des mittleren arteriellen Blutdrucks 36

Abb. 3.9 Boxplots zum Verlauf der Herzfrequenz 37

Abb. 3.10 Boxplots zum Verlauf des arteriellen pH-Wertes 38

Abb. 6.1 Boxplots zum Verlauf der Adrenalin-Dosierung.

Aufspaltung in $\mathrm{PCl}=0$ und $\mathrm{PCl}=1$

Abb. 6.2 Boxplots zum Verlauf der Dobutamin-Dosierung.

Aufspaltung in $\mathrm{PCl}=0$ und $\mathrm{PCl}=1$

Abb. 6.3 Boxplots zum Verlauf des mittleren arteriellen Blutdruckes.

Aufspaltung in $\mathrm{PCl}=0$ und $\mathrm{PCl}=1$

Abb. 6.4 Boxplots zum Verlauf der Herzfrequenz.

Aufspaltung in $\mathrm{PCl}=0$ und $\mathrm{PCl}=1$

Abb. 6.5 Boxplots zum Verlauf des arteriellen $\mathrm{pH}$-Wertes.

Aufspaltung in $\mathrm{PCl}=0$ und $\mathrm{PCl}=1$ 


\section{II.b Tabellenverzeichnis}

Tab. 3.1 Basisdaten des Respirations-Kollektivs 19

Tab. 3.2 Kühlungsparameter des Respirations-Kollektivs 21

Tab. 3.3 Beatmungsparameter und Blutgaswerte bei Ankunft auf Intensivstation. 22

Tab. 3.4 Beatmungsparameter und Blutgaswerte bei Erreichen von $34^{\circ} \mathrm{C}$ 22

Tab. 3.5 Beatmungsparameter und Blutgaswerte bei Erreichen von $33^{\circ} \mathrm{C} \ldots \ldots \ldots \ldots \ldots . . . . . .23$

Tab. 3.6 Basisdaten des kardialen Kollektivs 27

Tab. 3.7 Kühlungsparameter des kardialen Kollektivs

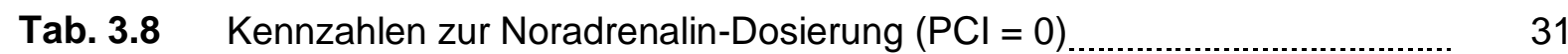

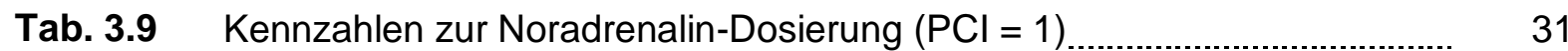

Tab. 3.10 Kennzahlen zur Adrenalin-Dosierung

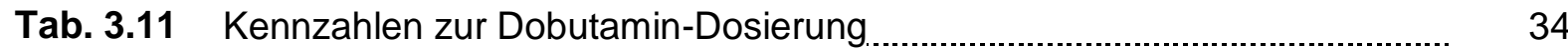

Tab. 3.12 Kennzahlen zum mittleren arteriellen Blutdruck ..........................................

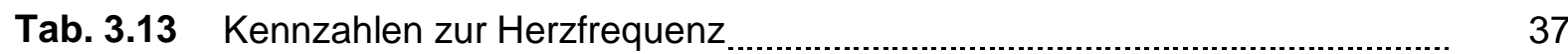

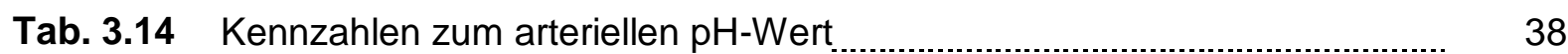

Tab. 6.1 Nichtparametrische ANOVA mit Paarvergleichen zu den Parametern $\mathrm{PaO}_{2}, \mathrm{FiO}_{2}$, PEEP und $\mathrm{PaO}_{2} / \mathrm{FiO}_{2}$

Tab. 6.2 Nichtparametrische ANOVA zu den Parametern Noradrenalin, Adrenalin, Dobutamin, mittlerem arteriellen Blutdruck, Herzfrequenz und arteriellem $\mathrm{pH}-$ Wert

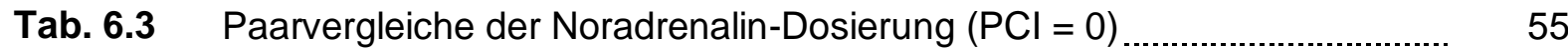

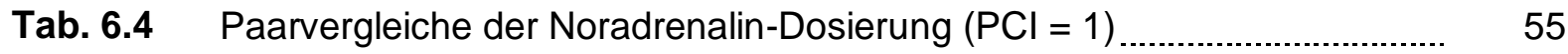

Tab. 6.5 Paarvergleiche der Adrenalin-Dosierung ...................................................... 55

Tab. 6.6 Paarvergleiche des mittleren arterielle Blutdrucks ........................................ 55

Tab. 6.7 Paarvergleiche der Herzfrequenz $\ldots$

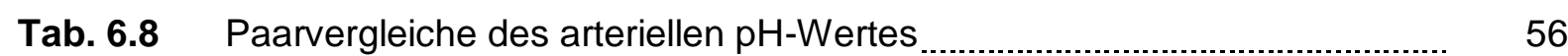

Tab. 6.9 Kennzahlen zur Adrenalin-Dosierung $(\mathrm{PCl}=0) \ldots$

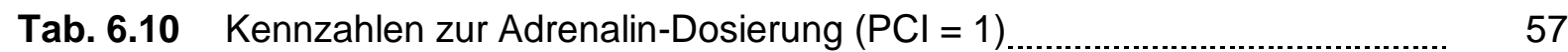

Tab. 6.11 Kennzahlen zur Dobutamin-Dosierung $(\mathrm{PCl}=0) \ldots$

Tab. 6.12 Kennzahlen zur Dobutamin-Dosierung $(\mathrm{PCl}=1) \ldots$

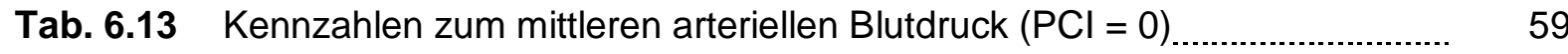

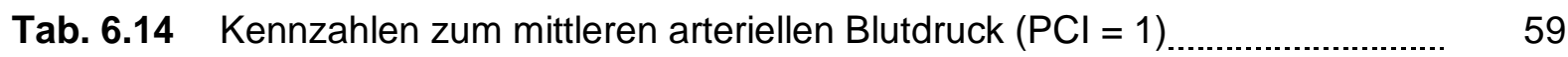

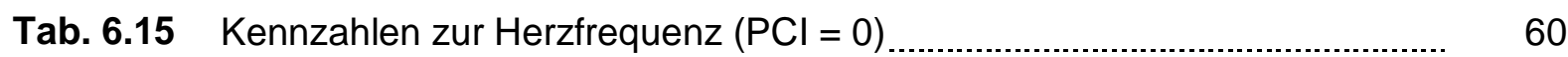

Tab. 6.16 Kennzahlen zur Herzfrequenz $(\mathrm{PCl}=1) \ldots$

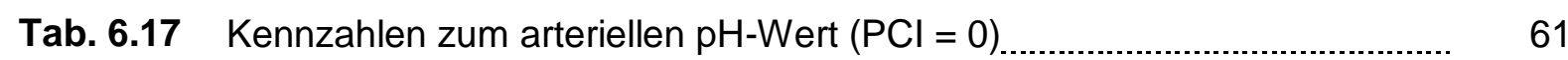

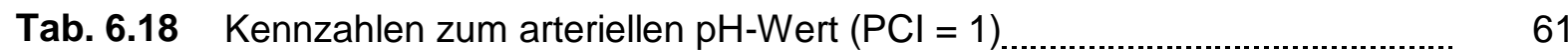




\section{Einleitung}

\subsection{Herzkreislaufstillstand und kardiopulmonale Reanimation - Relevanz und Häufigkeit}

Herzerkrankungen, allen voran die Chronisch ischämische Herzkrankheit, gefolgt von akutem Myokardinfarkt und Herzinsuffizienz, sind laut WHO die häufigsten Todesursachen in Deutschland (Statistisches Bundesamt, 2010). In ganz Europa liegt die Inzidenz eines plötzlichen Herz-Kreislauf-Stillstandes (HKS) bei 37-97 pro 100.000 Einwohnern und Jahr (Vreede-Swagemakers et al., 1997; Atwood et al., 2005). Auf eine Gesamtbevölkerung der Europäischen Union von mittlerweile 495 Millionen Menschen hochgerechnet, sind dies bis zu 480.000 HKS pro Jahr (Europäische Union, 2010).

Ein HKS zeichnet sich durch eine Unterbrechung des Blutflusses aufgrund eines Ausfalls der Pumpfunktion des Herzens unterschiedlicher Genese aus. Infolgedessen kommt es zu einem Sistieren der Atmung. Es handelt sich somit um eine akut lebensbedrohliche Situation mit der Gefahr des Hirntods (Arnold and Pschyrembel, 2013). Aber selbst nach primär erfolgreicher kardiopulmonaler Reanimation (CPR) mit Wiederherstellung eines Spontankreislaufs (ROSC), der nur in weniger als 50 Prozent (\%) der Fälle erreicht werden kann (Böttiger et al., 1999), ist die Überlebensrate seit sechs Jahrzehnten konstant gering. So konnten in der ersten großen Studie zur CPR aus dem Jahr 1953 ungefähr 50\% der Patienten lebend entlassen werden (Stephenson et al., 1953). In einer Studie der National Registry of Cardiopulmonary Resuscitation (NRCPR) aus dem Jahre 2006 mit 19.819 ROSC-Patienten verstarben im weiteren Behandlungsverlauf im Krankenhaus 67\% der Patienten (Nadkarni et al., 2006). Momentan wird die Überlebensrate, aufgeschlüsselt nach HKS außerhalb des Krankenhauses (OHCA), in einer Metaanalyse aus dem Jahr 2010 zusammengefasst mit 6,7-8,4\% angegeben (Sasson et al., 2010); innerhalb des Krankenhauses (IHCA) mit 18\% (Peberdy et al., 2003; Ehlenbach et al., 2009). Die Ein-Jahres-Überlebensrate eines HKS nach erfolgreicher Entlassung beträgt zwischen 2-6\% (Pell et al., 2003). Ursachen für diese hohe Letalität sind die Grunderkrankung selbst, die zu dem HKS geführt hat, und Schäden an verschiedenen Organsystemen, die durch den HKS und die anschließende Reperfusion verursacht werden. Letztere Phänomene werden unter 
dem Begriff des Postreanimationssyndroms subsummiert (Neumar et al., 2008). Hierbei ist vor allem (v.a.) das Ausmaß des hypoxischen Hirnschadens ausschlaggebend für die Prognose der Patienten (Laver et al., 2004).

Im Verhältnis zu der geringen Überlebensrate sind nicht zuletzt auch die Behandlungskosten eines Reanimationspatienten von großer ökonomischer Bedeutung. In einer 2004 veröffentlichten Studie aus Oslo, Norwegen, zeigte sich, dass die Behandlungskosten eines Reanimationspatienten vom Einsatz des Rettungsdienstes über die Krankenhausbehandlung bis hin zu Rehabilitationsmaßnahmen insgesamt um die 40.500 Euro betragen (Naess and Steen, 2004). Eine stetige Verbesserung des Reanimationsmanagements und der (Intensiv-) Therapie nach Reanimation ist daher oberstes Ziel, um das Outcome der Patienten zu verbessern und die Kosten zu reduzieren.

\subsection{Postreanimationssyndrom}

Bei einem HKS kommt es zu einer Ischämie des gesamten Körpergewebes. Besonders die lebenswichtigen Organe mit einem hohen Sauerstoff $\left(\mathrm{O}_{2}\right)$-Verbrauch, insbesondere das Gehirn, reagieren sehr empfindlich auf den Verlust der $\mathrm{O}_{2^{-}}$und Substratversorgung wie Adenosintriphosphat (ATP). Wird nicht schnellstmöglich eine Reperfusion des (Gehirn-) Gewebes erzielt, kommt es zur irreversiblen Schädigung (Arnold and Pschyrembel, 2013). Doch auch durch die erfolgreiche Wiederherstellung einer Blutzirkulation mit Reoxygenierung kann es zu unterschiedlichen Schädigungsprozessen kommen.

Erstmals prägte in diesem Zusammenhang Negovsky im Jahr 1988 den Begriff der „postresuscitation disease“ (Postreanimationskrankheit). Hierunter werden verschiedene pathophysiologisch und multiorganisch ablaufende Prozesse nach erfolgreicher CPR subsummiert (Negovsky, 1988).

Heute wird analog dazu der Begriff „Postreanimationssyndrom“ (PCAS) verwendet. Im Jahre 2008 erschien von dem International Liaison Committee on Resuscitation (ILCOR) ein consensus statement über die Epidemiologie, Pathophysiologie, Behandlung und Prognose des PCAS. Demnach kann die Pathophysiologie in vier Kernkomponenten eingeteilt werden (Neumar et al., 2008): 


\section{a) Postreanimations-Hirnschädigung}

In einer Studie aus dem Jahr 2004 konnte gezeigt werden, dass die Hirnschädigung eine sehr häufige Todesursache nach primär erfolgreicher Reanimation ist. So verstarben 68\% der Patienten nach prähospitaler CPR und 23\% nach intrahospitaler CPR im weiteren Behandlungsverlauf im Krankenhaus an neurologischen Schäden (Laver et al., 2004).

Neben der primären Hirnschädigung durch die Hypoxie während des HKS kommt es nach ROSC zu einer weiteren sekundären Hirnschädigung. Durch die Entstehung freier Sauerstoff-Radikale, einer gestörten Calcium-Homöostase, der Aktivierung von Zelltod-vermittelnden Signalwegen, pathologischer Protease-Kaskaden und exzitotoxischem Zelltod kommt es zu einer geschädigten zerebrovaskulären Autoregulation, einem Hirnödem und einer postischämischen Neurodegeneration, die innerhalb von Stunden bis Tagen nach ROSC entstehen können (Neumar et al., 2008).

\section{b) Postreanimations-Myokarddysfunktion}

Auch eine myokardiale Dysfunktion nach erfolgreicher CPR ist eine häufige Todesursache im weiteren klinischen Behandlungsverlauf. Dies konnte in mehreren Studien nachgewiesen werden (Heyndrickx et al., 1975; Ruiz-Bailén et al., 2005).

Pathophysiologisch müssen zwei verschiedene Ursachen der myokardialen Dysfunktion nach Wiederherstellung des Spontankreislaufs unterschieden werden. Zum einen ist in vielen Fällen ein Akutes Koronarsyndrom (ACS) ursächlich für den Herzkreislaufstillstand. Nach erfolgreicher Reanimation ist der Verschluss oder die kritische Stenose der Koronararterie noch nicht behoben, so dass die lokale Ischämie zu einer verminderten Kontraktilität des betroffenen Myokardareals führt. Eine globale myokardiale Dysfunktion wird jedoch auch bei Patienten beobachtet, deren Herzkreislaufstillstand nicht durch ein ACS ausgelöst wurde (Voicu et al., 2012). Ursächlich für diese myokardiale Dysfunktion ist die globale Minderperfusion des Myokards während des Kreislaufstillstandes. Es kommt zu einem sogenannten myokardialen stunning (aus dem Englischen „betäuben“). Dies ist eine potentiell reversible Funktionsstörung der Kontraktilität des Herzens, die auch nach Wiederherstellung der Koronarreperfusion zunächst fortbesteht (Heyndrickx et al., 1975; Braunwald and Kloner, 1982). Bei einem ACS zeigt sich dieses Phänomen lokal, nach einem HKS global. Darüber hinaus kommt es in der Reperfusionsphase 
zu einer Freisetzung von Tumor-Nekrose-Faktor (TNF)- $\alpha$ und anderen Entzündungsmediatoren, die negative Auswirkungen auf die Myozytenfunktion haben (Niemann et al., 2004; Schneider et al., 2012).

In einer Studie von Laurent et al. zeigte sich, dass es bei $54,5 \%$ der Patienten mit OHCA nach einer Median-Zeit von 6,8 Stunden zu hämodynamischer Instabilität mit Katecholaminpflichtigkeit kam. Es konnte ein erhöhter linksventrikulärer enddiastolischer Druck (LVEDP), eine reduzierte Ejektionsfraktion (EF), ein reduzierter Herzindex $(\mathrm{Cl})$ mit Abnahme von Herzzeitvolumen (HZV) und Schlagvolumen (SV) sowie ein niedriger mittlerer arterieller Blutdruck (MAP) nachgewiesen werden (Laurent et al., 2002). Histologisch nachweisbare Nekroseareale sind selten. Allerdings ist die Reversibilität der Funktionsstörung stark abhängig von der Dauer des HKS (Schneider et al., 2012).

\section{c) Systemischer Ischämie-Reperfusions-Schaden}

Wie oben bereits erwähnt, sind sowohl die Ischämie als auch die Reperfusion eigenständige Ursachen der Gewebeschädigung.

Während der Ischämie-Phase kommt es aufgrund der Hypoxie zu einer LaktatAnsammlung mit daraus resultierender metabolischer Azidose und Verminderung der ATP-Gewinnung (anaerobe Glykolyse).

Nach Reperfusion des Gewebes kommt es zu einer systemischen Entzündungsreaktion des gesamten Organismus, auch Systemisches inflammatorisches Response-Syndrom (SIRS) genannt. Dabei konnte die vermehrte Freisetzung von Zytokinen, v.a. Interleukin(IL)-6, IL-8 und IL-10 sowie TNF- $\alpha$ und löslichem TNF-Rezeptor II (sTNFRII) nachgewiesen werden (Adrie et al., 2002). Es kommt zur Einwanderung neutrophiler Granulozyten durch das Endothel in das Gewebe, was zur Gewebe- und Endothelschädigung führt (Gando et al., 2000). Diese Endothelschädigung ist eine wichtige Ursache für eine erhöhte Gefäßpermeabilität mit Extravasation und Aktivierung der Blutgerinnung. Es kommt zu einer Dysbalance zwischen überschießender Gerinnungskaskade und unzureichender endogener Fibrinolyse (Böttiger et al., 1995). Mikrothromben sind die Folge, die zu einer Störung der Mikrozirkulation führen („no-reflow“-Phänomen). Trotz adäquater Wiederherstellung des Kreislaufs sind nicht reperfundierte Areale in der Mikrozirkulation, v.a. des Gehirns, nachweisbar (Fischer and Hossmann, 1995). Ebenso kommt es zu einer verminderten Vasoregulation im Sinne einer 
systemischen Vasodilatation mit relativer Hypovolämie und Blutdruckabfall (Neumar et al., 2008; Schneider et al., 2012).

\section{d) Persistierende pathologische Prozesse, die den HKS verursacht haben}

Auch die Ursache des HKS, also die Grunderkrankung selber, kann zur Verstärkung der pathologischen Prozesse des PCAS beitragen oder anders herum durch das PCAS verstärkt werden. Hauptursache sind hierbei kardiovaskuläre Erkrankungen (v.a. ACS); aber auch pulmonale oder thrombembolische Erkrankungen sowie Infektionen können ursächlich sein. Sie erfordern eine zusätzliche, speziell auf das Krankheitsbild abgestimmte Behandlung (Neumar et al., 2008).

In der Zusammenschau ist festzuhalten, dass die verschiedenen Komponenten der Pathophysiologie des PCAS sehr komplex sind, sich gegenseitig bedingen und verstärken. Daher ist die Therapie kompliziert und ihre Optimierung ständig Gegenstand der Forschung, um die Prognose der Patienten zu verbessern.

\subsection{Milde therapeutische Hypothermie}

Es sei kurz erwähnt, dass es Überlegungen gibt, die Bezeichnung „Milde therapeutische Hypothermie“ durch den Begriff „Targeted Temperatur Management" (Zieltemperatur-Management) zu ersetzen, da „therapeutisch“ eine positiv zu wertende Bezeichnung ist, die nicht in jedem Fall nachgewiesen werden kann (Nunnally et al., 2011).

\subsubsection{Einsatz nach kardiopulmonaler Reanimation}

Für Patienten nach HKS konnte als bisher einzige Therapieoption zur signifikanten Verbesserung der Überlebensrate bzw. des neurologischen Outcomes in zwei großen randomisierten Studien ein positiver Einfluss der milden therapeutischen Hypothermie (MTH) nachgewiesen werden.

Eingeschlossen in die Studien wurden komatöse Patienten mit ROSC nach beobachtetem OHCA und Kammerflimmern (KF) als erstem abgeleiteten Herzrhythmus (HR) (The Hypothermia after Cardiac Arrest Study Group, 2002; Bernard et al., 2002). 
Die 2002 veröffentlichte Studie von Bernard et al. wurde in Melbourne, Australien durchgeführt. Es wurden 77 Patienten eingeschlossen; 43 Patienten wurden mit MTH behandelt, 39 Patienten unter normothermen Bedingungen. Die Hypothermie-Gruppe wurde für 12 Stunden (h) auf $33^{\circ} \mathrm{C}$ (Grad Celsius) Körperkerntemperatur (KKT) heruntergekühlt. 21 Patienten dieser Gruppe (49\%) konnten entlassen werden und hatten ein signifikant besseres neurologisches Outcome im Vergleich zu 9 Patienten in der Kontroll-Gruppe (26\%).

In die ebenfalls 2002 veröffentlichte europäische Multicenter-Studie der „Hypothermia After Cardiac Arrest Study Group“, auch HACA-Studie genannt, wurden 273 Patienten eingeschlossen. 137 Patienten wurden bei MTH von $34-32^{\circ} \mathrm{C}$ KKT über $24 \mathrm{~h}$ behandelt, 138 Patienten bei Normothermie. In der MTH-Gruppe hatten 75 Patienten (55\%) ein gutes neurologisches Outcome im Vergleich zu 54 Patienten (39\%) in der Normothermie-Gruppe. Auch die Sterberate war in der MTHGruppe signifikant geringer als in der Kontrollgruppe mit $41 \%$ zu 55\%.

Dies veranlasste im Oktober 2002 das ILCOR dazu, eine Stellungnahme herauszugeben. Es wurde empfohlen, alle komatösen Erwachsenen nach durch Kammerflimmern induziertem OHCA und erfolgreicher Reanimation mit einer milden therapeutischen Hypothermie von $34-32{ }^{\circ} \mathrm{C}$ über 12-24 Stunden zu behandeln (Nolan et al., 2003).

Im Winter 2005 veröffentlichten die amerikanischen und europäischen Fachgesellschaften neue Leitlinien zur Reanimation, in welche diese Empfehlungen aufgenommen wurden. Ebenso wurde festgehalten, dass auch Patienten mit nicht defibrillierbarem ersten Herzrhythmus oder Patienten nach intrahospitaler CPR von einer MTH profitieren könnten (Nolan et al., 2005; International Liaison Committee on Resuscitation, 2005). Die aktuell gültigen Leitlinien zur Reanimation aus dem Jahr 2010 empfehlen die schnellstmögliche Einleitung einer MTH bei allen komatösen Überlebenden eines HKS, unabhängig davon, ob der initiale HR defibrillierbar war oder nicht. Auch die Kühlgeschwindigkeit bis zum Erreichen der Zieltemperatur sollte so rasch wie möglich sein und nach erfolgter Hypothermiephase sind Hyperthermien zu vermeiden, da sie zu einem verschlechterten Outcome führen können (Deakin et al., 2010).

Trotz eindeutiger Datenlage und Empfehlung in den Leitlinien werden in Europa und den USA nur circa 30\% der reanimierten Patienten einer MTH zugeführt (Abella et al., 2005; Merchant et al., 2006; Wolfrum et al., 2007). So konnte in einer 2006 von 
Wolfrum et al. erschienenen Studie gezeigt werden, dass nur auf 23,5\% der deutschen Intensivstationen die MTH angewendet wird. Als Gründe hierfür wurden v.a. fehlende Erfahrung mit bzw. fehlende Informationen/Daten über MTH sowie Schwierigkeiten bezüglich der technischen Handhabung der speziellen Kühlungsgeräte und deren Kosten genannt (Abella et al., 2005; Merchant et al., 2006; Wolfrum et al., 2007).

\subsubsection{Effekte der milden therapeutischen Hypothermie}

Beim gesunden Menschen liegt die Körperkerntemperatur, definiert als Bluttemperatur im Hypothalamus, in einem engen Bereich von $36,6 \pm 0,38^{\circ} \mathrm{C}$, was als Normothermie bezeichnet wird (Brüx et al., 2005). Eine Hypothermie beginnt ab einer KKT von $<35^{\circ} \mathrm{C}$. Sie wird eingeteilt in drei Gruppen. Bei der milden Hypothermie handelt es sich um eine Reduktion der KKT auf $35-32^{\circ} \mathrm{C}$. Eine moderate Hypothermie liegt bei einer KKT von $32-30^{\circ} \mathrm{C}$ vor und eine tiefe Hypothermie bei $<30^{\circ} \mathrm{C}$ KKT (Nolan et al., 2005). Nach anfänglichen Versuchen mit tiefer Hypothermie in den Fünfzigerjahren, die mit potenziell schweren Nebenwirkungen verbunden waren, zeigten Versuche in den Achtziger- und Neunzigerjahren schließlich, dass die milde Hypothermie nebenwirkungsärmer und daher als Therapieoption geeignet ist (Popp et al., 2005).

Oberstes Therapieziel bei Reanimationspatienten ist es, Überleben zu sichern und das neurologische Outcome zu verbessern. Dabei steht bei der MTH die neuroprotektive Wirkung im Vordergrund.

Effekte auf das Gehirn: Der positive Effekt der milden therapeutischen Hypothermie $\left(34-32^{\circ} \mathrm{C}\right)$ besteht zunächst in einem allgemein herabgesetzten Zellmetabolismus. So konnte bereits 1954 gezeigt werden, dass es zu einer 5-7 prozentigen Reduktion des Sauerstoff- und Glukoseverbrauchs der (Gehirn-) Zellen bei Temperaturreduktion um $1^{\circ} \mathrm{C}$ kommt (Rosomoff and Holaday, 1954; Popp et al., 2005). Zum anderen werden im Rahmen des PCAS entstehende multifaktorielle biochemische und physiologische Mechanismen unterdrückt, welche nach Reperfusion eine zusätzliche Zellschädigung verursachen könnten. So kommt es durch die erloschene ATP-Versorgung des Gehirns zu einer Akkumulation von Glutamat, einem präsynaptischen exzitatorischen Neurotransmitter, der durch den 
Energiemangel nicht mehr abgebaut werden kann. Dies führt zur Daueraktivierung seines Rezeptors und daraus resultierend zu einer Verschiebung von Kalzium $\left(\mathrm{Ca}^{2+}\right)$ aus dem extra- in den intrazellulären Raum und damit zu einer $\mathrm{Ca}^{2+}$-Überladung der Zelle. Ausbildung freier Radikale und andere zellschädigende Mechanismen mit exzitotoxischem Zelltod sind die Folge (Szydlowska and Tymianski, 2010). Tierexperimentell konnte nachgewiesen werden, dass die MTH hier eine Reduktion von Glutamat bewirkt und damit die zellschädigenden Mechanismen inhibiert (Hachimi-Idrissi et al., 2004).

Effekte auf das Myokard: Auch die Kontraktilität von Kardiomyozyten ist von der Temperatur abhängig. Bereits im Jahr 1897 beschrieb Langendorff erstmals einen Zusammenhang zwischen Myokardfunktion und Temperaturunterschieden (Langendorff, 1897). Auch in neueren Studien am menschlichen sowie am Schweinemyokard konnte festgestellt werden, dass die MTH positiv inotrope Effekte besitzt, so dass die Kontraktionskraft des Arbeitsmyokards zunimmt.

Physiologisch spielt die intrazelluläre $\mathrm{Ca}^{2+}$-Konzentration des Arbeitsmyokards eine wichtige Rolle während des Kontraktionsvorgangs. Die Kontraktionskraft (Inotropie) der Kardiomyozyten hängt dabei von der Höhe der $\mathrm{Ca}^{2+}$-Konzentration im Zytosol ab (Klinke et al., 2010). Die hypothermiebedingte Zunahme der Kontraktilität scheint jedoch weder durch einen signifikant erhöhten $\mathrm{Ca}^{2+}$-Einstrom von extrazellulär, noch durch einen signifikant erhöhten $\mathrm{Ca}^{2+}$-Ausstrom aus dem SR bedingt zu sein, auch wenn die Aktionspotenzial(AP)-Dauer verlängert ist. Vielmehr scheint es sich um eine erhöhte Empfindlichkeit der Myofilamente gegenüber $\mathrm{Ca}^{2+} \mathrm{zu}$ handeln (Churcott et al., 1994; Weisser et al., 2001; Jacobshagen et al., 2010).

Jedoch gibt es auch Studien, die in diesem Zusammenhang zu widersprüchlichen Ergebnissen kommen (Goetzenich et al., 2009).

Effekte auf die Hämodynamik: In der Studie von Weisser ließ sich ebenfalls ein steigernder Effekt der MTH auf die hämodynamischen Parameter anästhesierter Schweine zeigen. Obwohl die HF im Kühlungsverlauf sank, erhöhte sich das SV so stark, dass das HZV insgesamt signifikant anstieg. Systemischer und pulmonaler Gefäßwiderstand blieben dabei unverändert. Damit hat die MTH einen direkten positiv inotropen Effekt auf die Hämodynamik (Weisser et al., 2001). Auch hier kommen andere Studien zu gegensätzlichen Ergebnissen. So wurde dargestellt, 
dass Hypothermie zu einem verringerten $\mathrm{HZV}$ und $\mathrm{Cl}$ sowie zu einem erhöhten Katecholaminbedarf führen kann (Bernard et al., 2002; Goetzenich et al., 2009).

Andere Effekte: Nicht zuletzt konnten anti-inflammatorische und anti-apoptotische Eigenschaften der MTH gezeigt werden (Xu et al., 2002; Meybohm et al., 2010). So kommt es unter MTH zu einer verminderten Induktion von Zytokinen, insbesondere IL-1, IL-6, IL-10 und TNF-a sowie zur Inhibierung von Caspasen, spezifischer Proteasen, die im Rahmen des PCAS zu einer Aktivierung von Zelltod-vermittelnden Signalwegen führen.

Doch neben den positiven Effekten der MTH sind auch damit einhergehende Nebeneffekte bzw. Komplikationen beschrieben.

In der HACA-Studie aus 2002 konnte dabei allerdings gezeigt werden, dass die Komplikationsrate generell unter milder therapeutischer Hypothermie nicht signifikant (n.s.) erhöht ist gegenüber der bei Intensivtherapie unter Normothermie (73\% gegenüber 70\%). Und auch die Komplikationen selbst variieren nicht signifikant zwischen MTH- und Normothermie-Gruppe (The Hypothermia after Cardiac Arrest Study Group, 2002).

Komplikationen durch die MTH: Zu den häufigsten Komplikationen gehören laut einer Studie aus dem Jahr 2009 von Nielsen et al. mit 986 eingeschlossenen Patienten Infektionen, allen voran Pneumonien (41\%) bis hin zur Sepsis (4\%), Hyperglykämien (37\%) -eine verminderte Insulin-Sekretion unter Hypothermie konnte bereits 1970 beschrieben werden (Curry and Curry, 1970)- Herzrhythmusstörungen (33\%), Krampfanfälle (24\%) sowie Elektrolytentgleisungen (Hypophosphatämie 19\%, Hypokaliämie 18\% und Hypomagnesiämie 18\%) (Nielsen et al., 2009).

Komplikationen bei der technischen Durchführung der MTH: Eine Metaanalyse von 41 klinischen Studien zwischen 1997 und 2010 stellte dar, dass die durch den Einsatz von technischen Hilfsmitteln zur Kühlung verursachte aggregierte Komplikationsrate bei $1 \%$ lag (29 von 3.133 Patienten). Dabei kam es unter Zuhilfenahme von Kühlungskathetern zu drei Fällen von Blutung, 8 Infektions-Fällen und 10 Fällen von tiefer Beinvenenthrombose. Unter dem Gebrauch von kalten Infusionen kam es in 8 Fällen zu einem Lungenödem (Holzer, 2010). 


\subsubsection{Kühlungsmethoden}

Es existieren die verschiedensten Techniken zur Induktion und Aufrechterhaltung einer MTH. Dabei kann man zunächst zwischen Oberflächenkühlung und intravasaler Kühlung unterscheiden. Häufig werden verschiedene Kühlungsformen kombiniert.

Zu Formen der Oberflächenkühlung gehören als simpelste Möglichkeit das Auflegen von Eispacks auf Kopf, Hals, Leiste und Extremitäten der Patienten, aber auch der Einsatz von Kühldecken und -matten oder Kaltluftzufuhr. Diese Methoden sind kostengünstig und zur Induktion einer Hypothermie einfach einzusetzen. Problematisch ist jedoch, dass es oft nur zu einer langsamen Reduktion der KKT kommt und sich die Aufrechterhaltung einer Zieltemperatur von $32-34^{\circ} \mathrm{C}$ als kompliziert darstellt (Hoedemaekers et al., 2007).

Demgegenüber gibt es die Möglichkeit der intravasalen Kühlung, zum einen über endovaskuläre Kühlungssysteme, zum anderen durch die intravenöse Volumentherapie mittels kalter Infusionen.

Erstere sind äußerst effektiv bezüglich schneller Reduktion der KKT auf die Zieltemperatur und genauer Temperaturkontrolle. Die Systeme sind jedoch invasiv und kostspielig in Erwerb und Betrieb (ca. 900 Euro pro Patient) (Schneider et al., 2012). Die Kühlung erfolgt über ein Kathetersystem, welches meist in die Vena femoralis eingebracht wird. In dem Katheter zirkuliert permanent kalte Flüssigkeit, durch die das vorbeifließende Blut abgekühlt und systemisch weitergeleitet wird (Hoedemaekers et al., 2007). Längst nicht jedes Krankenhaus verfügt über solche invasiven Kühlungssysteme.

Die Kühlung mittels $2-4^{\circ} \mathrm{C}$ kalter $0,9 \%$ iger Kochsalz-Lösung (NaCl-Lösung) oder anderer kristalloider Infusionen kann schon präklinisch, spätestens aber in jedem Krankenhaus begonnen werden. Empfohlen ist eine Volumengabe von 30 Milliliter/Kilogramm ( $\mathrm{ml} / \mathrm{kg}$ ) Körpergewicht (KG) appliziert über 30 Minuten (min). Dies wären bei einem $70 \mathrm{~kg}$-Patienten $2.100 \mathrm{ml}$ Flüssigkeit. Eine Kühlung auf die Zieltemperatur ist schneller möglich als bei der Oberflächenkühlung. Somit stellt sie eine effektive, in jedem Krankenhaus einfach zu handhabende und kostengünstige Möglichkeit zur Kühlung dar (Bernard et al., 2003; Jacobshagen et al., 2009). 
Abschließend ist festzuhalten, dass bisher keine Überlegenheit einer Kühlungsmethode bezüglich des Outcomes der Patienten gezeigt werden konnte und dementsprechend auch keine konkreten Empfehlungen zur Kühlungsmethode bestehen.

\subsubsection{Weitere Therapiestrategien}

Neben intensivmedizinischer Standardtherapie der Grunderkrankung erhalten generell alle Patienten, die mit MTH behandelt werden, eine adäquate Analgesie und Sedation, sowie eine Muskelrelaxation, um Kältezittern zu vermeiden. Dieses kann zu einem erhöhten Sauerstoffverbrauch der Zellen, einer erhöhten Herzfrequenz (HF) und Atemanstrengung führen und durch die mit dem Zittern verbundene Erhöhung der Körpertemperatur der Kühlungsphase entgegenwirken (Holzer, 2010).

Atmung: Im Rahmen der systemischen Entzündungsreaktion nach Reanimation entwickeln viele Patienten ein Akutes Atemnotsyndrom (ARDS) (Ranieri et al., 2012). Daher ist eine optimale, lungenprotektive Beatmung sehr wichtig. Die Zielwerte der Blutgase sollten in physiologischen Bereichen liegen bei einem arteriellen Kohlendioxid-Partialdruck $\left(\mathrm{PaCO}_{2}\right)$ von 40 Millimeter-Quecksilbersäule $(\mathrm{mmHg})$ sowie einer arteriellen Sauerstoffsättigung $\left(\mathrm{SaO}_{2}\right)$ zwischen $94-96 \%$ (Balan et al., 2006; Neumar et al., 2008). Eine Hyperventilation stellt dabei eine Gefahr für eine zusätzliche zerebrale Ischämie dar, da die aus einer Hyperventilation resultierende Hypokapnie zu einer zerebralen Vasokonstriktion führt. Eine Hyperkapnie andererseits würde zu einer Vasodilatation mit einem erhöhten Risiko für ein Hirnödem führen (Buunk et al., 1997).

Zur Erreichung dieser Zielwerte sollten der positive endexspiratorische Druck (PEEP), die inspiratorische Sauerstoffkonzentration $\left(\mathrm{FiO}_{2}\right)$ und der Beatmungsspitzendruck (Pmax) engmaschig angepasst werden.

Das Atemzugvolumen sollte $6 \mathrm{ml} / \mathrm{kg} \mathrm{KG}$ nicht übersteigen. Es sollte darauf geachtet werden, dass es nicht zu einer Hyperoxygenierung kommt, da gezeigt werden konnte, dass dies zu einem verschlechterten neurologischen Outcome führen kann (Balan et al., 2006).

Als ein Maß für die Schädigung der Lunge kann zusätzlich der Horovitz-Quotient herangezogen werden. Er berechnet sich aus dem Quotienten von arteriellem 
Sauerstoff-Partialdruck $\left(\mathrm{PaO}_{2}\right)$ und $\mathrm{FiO}_{2}$. Bei Lungengesunden liegt er um 500 $\mathrm{mmHg}$.

Hämodynamik: Es sollte eine adäquate Unterstützung der Hämodynamik erfolgen, da die meisten Patienten im Rahmen des PCAS zu hämodynamischer Instabilität neigen (Laurent et al., 2002). Die Symptome des Postreanimationssyndroms sind denen der Sepsis sehr ähnlich, weshalb in einer ersten Studie von Gaieski et al. (2009) untersucht wurde, wie sich ein leitliniengerechter Algorithmus in der SepsisTherapie, die "early goal-directed therapy", bei Anwendung auf Reanimationspatienten auswirkt. Die Therapie beginnt dabei schnellstmöglich nach Diagnose eines SIRS mit instabiler Kreislaufsituation. Zielparameter sind ein zentraler Venendruck von 8-12 mmHg, ein MAP von $65-90 \mathrm{mmHg}$ und eine zentralvenöse $\mathrm{O}_{2}$-Sättigung von mehr als $70 \%$. Aufgrund dieses Algorithmus konnte die intrahospitale Mortalität von Patienten mit schwerer Sepsis signifikant gesenkt werden (Rivers et al., 2001). Unter zusätzlicher Anwendung der MTH bei Reanimationspatienten ergab sich in der Gaieski-Studie aus 2009 eine intrahospitale Mortalität von 50\% (9/18) im Vergleich zu 78\% (14/18) bei einer historischen Kontrollgruppe von Reanimationspatienten, allerdings ohne Anwendung der MTH ( $p$ $=0,15)$ (Gaieski et al., 2009).

Bei relativer Hypovolämie aufgrund von Vasodilatation und Extravasation im Rahmen des SIRS nach Reanimation sollte eine intravenöse Volumensubstitution erfolgen. Zudem sollten inotrope Medikamente oder Vasopressoren eingesetzt, sowie, falls indiziert, mittels mechanischer Unterstützung die Hämodynamik verbessert werden. Bei Patienten mit ACS sollte eine umgehende Herzkatheterdiagnostik mit gegebenenfalls perkutaner koronarer Intervention (PCI) eingeleitet werden (Neumar et al., 2008).

Es wird empfohlen, die Kreislaufsituation mittels Volumens und Katecholaminen so zu unterstützen, dass der MAP hoch genug ist, um eine adäquate Diurese von 1 $\mathrm{ml} / \mathrm{kg} \mathrm{KG} \mathrm{zu} \mathrm{ermöglichen} \mathrm{(MAP} \mathrm{in} \mathrm{der} \mathrm{Regel} \geq 65 \mathrm{mmHg}$ ) (Nolan et al., 2005). Da hohe Katecholamindosen bzw. prolongierte Katecholamingaben über einen erhöhten myokardialen $\mathrm{O}_{2}$-Verbrauch und Aktivierung von Apoptosemechanismen kardiotoxische Effekte haben, im Herzen proinflammatorisch wirken und schließlich auch über vermehrte Rhythmusstörungen (v.a. durch Elektrolytentgleisungen) die Letalität im kardiogenen Schock erhöhen können, sollte ihre Dosierung so gering und 
kurzfristig wie möglich gehalten werden. Dabei ist das unterschiedliche Wirkungsprofil zu beachten und der Einsatz der jeweiligen Situation anzupassen (Schwertz et al., 2004; Tamargo et al., 2011; Burchardi et al., 2011).

Mittels arterieller Blutgasanalyse (BGA) sollten Elektrolyte, Glukose und weitere Parameter kontrolliert werden, um Entgleisungen frühestmöglich vorzubeugen, und bei bakteriellen Infektionen eine Antibiotikatherapie nach Antibiogramm erfolgen (Holzer, 2010).

\subsection{Zielsetzung der Arbeit}

Die milde therapeutische Hypothermie ist seit 2005 fester Bestandteil der EuropeanResuscitation-Council (ERC)-Leitlinien. Es gibt verschiedenste Möglichkeiten der Kühlung wie Eispacks, Kühlzelt oder intravasale Kühlungssysteme. Die Kühlung mittels kalter Infusionen stellt eine effektive, in jedem Krankenhaus einfach zu handhabende und zusätzlich kostengünstige Möglichkeit dar. Aufgrund der eingeschränkten linksventrikulären Funktion (LV-Funktion) und der systemischen Entzündungsreaktion bei reanimierten Patienten könnte die Sorge bestehen, dass sich eine großvolumige intravasale Flüssigkeitszufuhr negativ auf die Beatmungssituation des Patienten auswirkt.

Zudem gibt es Hinweise darauf, dass die therapeutische Hypothermie neben dem verbesserten neurologischen Outcome auch einen direkten positiven Effekt auf die kardiale Funktion und Hämodynamik besitzt und zu einer Verringerung des exogenen Katecholaminbedarfs führt.

In dieser Arbeit wurden daher retrospektiv zwei Analyseansätze verfolgt. Zum einen wurden die möglichen Effekte einer Volumentherapie mittels kalter Infusionen auf die respiratorische Funktion bei Patienten nach primär erfolgreicher kardiopulmonaler Reanimation untersucht. Hierbei wurde die Frage geprüft, ob eine Erreichung der milden Hypothermie mittels Hochvolumentherapie zu einer Verschlechterung der respiratorischen Situation der Intensivpatienten führt.

Zum anderen wurde die Frage geprüft, ob die milde Hypothermie einen Einfluss auf die Dosierung der Katecholamintherapie und die Herz-Kreislauf-Funktion der Patienten aufweist. 


\section{Methoden}

\subsection{Patienteneinschluss}

In dieser Arbeit wurden retrospektiv die Daten von 337 Patienten analysiert, welche nach primär erfolgreicher Reanimation bei Herz-Kreislauf-Stillstand mit milder therapeutischer Hypothermie im Herzzentrum der Universitätsmedizin Göttingen (UMG) therapiert wurden. Eingeschlossen in die Studie wurden ausnahmslos alle Patienten zwischen Januar 2003 und Dezember 2009 ab dem 18. Lebensjahr, bei denen eine annähernd lückenlose Dokumentation des Kühlungs- und Wiedererwärmungszeitraums den Patientenakten zu entnehmen war. Insgesamt wurden in diesem Zeitraum 436 Patienten wegen Herz-Kreislauf-Stillstandes behandelt.

Ein Herz-Kreislauf-Stillstand wurde definiert als Bewusstlosigkeit aufgrund eines pulslosen Kreislaufzustandes und Fehlen einer Spontanatmung. ROSC (Wiederherstellung eines Spontankreislaufs) wurde definiert als Wiedererlangung eines palpablen Pulses. Mit Ausnahme von Trauma-Patienten wurden alle Patienten mit einem dokumentierten HKS, außerhalb oder innerhalb der Universitätsmedizin Göttingen, einbezogen. Der initiale Herzrhythmus wurde kategorisiert in Kammerflimmern (KF) oder -tachykardie, Asystolie und pulslose elektrische Aktivität (PEA). Alle Patienten wurden intensivmedizinisch entsprechend den ILCOR/ERCLeitlinien behandelt, wurden intubiert und erhielten einen zentralen Venenkatheter (ZVK). Es erfolgte eine kontinuierliche Aufzeichnung der invasiven Blutdruckmessung, Herzfrequenz und Pulsoxymetrie. Die Körperkerntemperatur wurde kontinuierlich mittels einer Temperatursonde in der Harnblase (FoleyKatheter) oder einer Temperatursonde in der Arteria pulmonalis mittels Pulmonalarterienkatheter (PA-Katheter) gemessen. Die Kreislaufstabilisation erfolgte differenziert mittels inotroper Medikamente (Adrenalin, Dobutamin, Levosimendan) oder Vasopressoren (Noradrenalin, Vasopressin) je nach Bedarf. Eine mechanische Unterstützung zur Stabilisierung des Kreislaufs, wie eine intraaortale Ballonpumpe (IABP) oder eine linksventrikulärere Mikroaxial-Pumpe $\left(\right.$ Impella $\left.^{\circledR}\right)$, wurden bei entsprechender Indikation bereits im Herzkatheter-Labor (HKL) implantiert. Ziel war die Aufrechterhaltung eines MAP von $80 \mathrm{mmHg}$. Die Dosierung der 
unterschiedlichen Katecholamine erfolgte nach Ermessen des verantwortlichen Arztes der kardiologischen Intensivtherapiestation (ITS) auf Grundlage des MAP, der HF sowie Befunden der Echokardiographie (Echo), der produzierten Urinmenge und anderer gewichtiger Grunderkrankungen. Es erfolgten engmaschige temperaturkorrigierte arterielle Blutgasanalysen. Hierauf basierend wurde die Beatmungstherapie anhand der Zielwerte eines arteriellen $\mathrm{PaCO}_{2}$ von $40 \mathrm{mmHg}$ sowie einer arteriellen Sauerstoffsättigung zwischen $94-96 \%$ gesteuert. Alle Patienten erhielten eine lungenprotektive Beatmung mit einem Tidalvolumen von $\leq 6$ $\mathrm{ml} / \mathrm{kg} \mathrm{KG}$ und einem Spitzendruck von $\leq 30$ Millibar (mbar). Die linksventrikuläre Ejektionsfraktion (LVEF) wurde bei Erreichen der Universitätsmedizin Göttingen mittels echokardiographischer Standarddarstellungen oder mittels Lävokardiographie ermittelt. Eine milde therapeutische Hypothermie über 24 Stunden wurde bei allen Patienten mit persistierender Bewusstlosigkeit nach ROSC eingeleitet. Nach Beurteilung des neurologischen Status erfolgte die Sedierung aller Patienten mittels Midazolam und Fentanyl oder Propofol und Sulfentanyl. Um ein Kältezittern zu verhindern, erfolgte eine Relaxation der Patienten mittels Pancuronium in einer Dosierung von 8 Milligramm $(\mathrm{mg})$ alle 2 Stunden während der 24-stündigen Kühlungsphase. Mit Eintreffen des Patienten in der Notaufnahme, dem HerzkatheterLabor oder auf der ITS erfolgte leitliniengerecht eine unmittelbare Einleitung der MTH. Alle Patienten wurden mittels verschiedener extrakorporalen und intravasaler Kühlungsmethoden behandelt, wobei auch verschiedene Kühlungsmethoden miteinander kombiniert wurden. So erhielten die Patienten eine intravenöse Volumentherapie mittels $2-4^{\circ} \mathrm{C}$ kalter $0,9 \%$ iger Kochsalz-Lösung ( $\mathrm{NaCl}$-Lösung) oder anderer Kristalloide und/oder wurden zusätzlich mit einem endovaskulären Kühlungssystem (CoolGard ${ }^{\circledR}$, Alsius) oder extrakorporaler Kühlungsmaßnahmen (Kühlzelt DeltaTherm ${ }^{\circledR}, \mathrm{KCl}$; Eispacks) versorgt. Auch während therapeutischer oder diagnostischer Untersuchungen wie $\mathrm{PCl}$ oder Computertomographie (CT) wurde die MTH eingeleitet bzw. fortgeführt. Nach Erreichen der Zieltemperatur von $33^{\circ} \mathrm{C}$ wurden die Patienten über 24 Stunden konstant bei dieser Temperatur gehalten, waren weiterhin sediert und relaxiert. Nach 24 Stunden wurde mit einer passiven oder aktiven kontrollierten Wiedererwärmung von $0,25-0,5^{\circ} \mathrm{C}$ pro Stunde begonnen. Die Sedierung wurde bei einer Körperkerntemperatur von $35^{\circ} \mathrm{C}$ gestoppt. Innerhalb von 7-14 Stunden sollte eine Ziel-Körperkerntemperatur von $36,5^{\circ} \mathrm{C}$ erreicht werden. 
Es erfolgte eine systematische Datenanalyse der Basisdaten, der Kühlungsparameter, und zu den jeweiligen Zeitpunkten der Menge der infundierten Flüssigkeit, der Körperkerntemperatur, der arteriellen BGA, der pulmonalen Funktion, hämodynamischer Parameter und echokardiographischer Ergebnisse. Da das Körpergewicht der Patienten oft unmittelbar nach dem Akutereignis unbekannt ist, werden die Adrenalin- und Noradrenalin-Dosierungen in Mikrogramm/Minute $(\mu \mathrm{g} / \mathrm{min}) \mathrm{zu}$ den definierten Zeitpunkten angegeben.

\subsubsection{Datenerhebung für die respiratorische Funktion}

Es wurden Daten zu den drei Zeitpunkten Ankunft auf ITS (Intensivtherapiestation), Kühlung auf $34^{\circ} \mathrm{C}$ und $33^{\circ} \mathrm{C}$ erhoben. Untersucht wurden die Menge an kaltem Volumen, welches infundiert wurde, der arterielle Sauerstoff-Partialdruck $\left(\mathrm{PaO}_{2}\right)$, die inspiratorische Sauerstoffkonzentration $\left(\mathrm{FiO}_{2}\right)$, der Horovitz-Quotient $\left(\mathrm{PaO}_{2} / \mathrm{FiO}_{2}\right)$ und der positive endexspiratorische Druck (PEEP). Ausgeschlossen von diesem Teil der Studie wurden 171 Patienten. Davon entfielen 65 Patienten aufgrund fehlender Kühlung mittels kalter Infusionen, 6 Patienten, welche vor Erreichen einer Körperkerntemperatur von $33^{\circ} \mathrm{C}$ verstarben, und 100 Patienten aufgrund unvollständiger Dokumentation der studienrelevanten Daten. Somit wurden 166 Patienten in diesen Teil der Analyse eingeschlossen.

\subsubsection{Datenerhebung für die kardiale Funktion und Hämodynamik}

Es wurden Daten zu den fünf Zeitpunkten Ankunft auf ITS, Kühlung auf $34^{\circ} \mathrm{C}$ und $33^{\circ} \mathrm{C}$, vor Beginn der Wiedererwärmung und bei Erreichen einer Temperatur von $36,5^{\circ} \mathrm{C}$ erhoben. Untersucht wurden die Dosierungen der Katecholamine Adrenalin, Noradrenalin (NA) und Dobutamin, die Herzfrequenz (HF), der mittlere arterielle Blutdruck (MAP), der arterielle pH-Wert sowie eine erfolgte perkutane koronare Intervention (PCl). Ausgeschlossen von diesem Teil der Studie wurden 178 Patienten. Davon entfielen 122 Patienten aufgrund unvollständiger Dokumentation der studienrelevanten Daten oder fehlender Gabe von Katecholaminen und 56 Patienten, welche während des Hypothermie- und Wiedererwärmungszeitraums verstarben. Somit wurden 159 Patienten in diesen Teil der Analyse eingeschlossen. 


\subsection{Statistische Analyse}

Die Auswertung der im Ergebnisteil beschriebenen Daten erfolgte mithilfe der Statistikprogramme STATISTICA (Version 10) und SAS (Version 9.3). Betrachtet wurden die in Kapitel 3 beschriebenen Patientenkollektive bezüglich respiratorischer Parameter $(n=166)$ ( $n$ : Anzahl der Patienten) und Herz-Kreislauf-Parameter ( $n=$ 159). Eingeschlossen wurden jeweils alle Patienten, die ein vollständiges Datenprofil zu allen definierten Temperaturpunkten aufwiesen.

Für die deskriptive Beschreibung der Basisdaten und Daten der therapeutischen Hypothermie wurden Mittelwert (MW) \pm Standardfehler des arithmetischen Mittels (SEM) berechnet. Die deskriptive Beschreibung der respiratorischen Daten, aufgeschlüsselt nach Temperaturpunkten, erfolgte mittels Median und Interquartilsabstand (IQR), die Beschreibung der kardialen Daten, aufgeschlüsselt nach Temperaturpunkten, mittels Median, MW und Spannweite (Spw.). Zur graphischen Darstellung wurden Boxplots und Scatterplots erstellt. Die Boxplots basieren auf den Kennzahlen Median, 25\%- und 75\%-Quantil (25\%-Q und 75\%-Q), Minimum (MIN) und Maximum (MAX). Ausreißer und Extremwerte wurden identifiziert, falls sie weiter als das 1,5-fache (Ausreißer) bzw. 2-fache (Extremwerte) der Länge der Box (25\%-75\%-Quantil) vom 25\%- bzw. 75\%-Quantil entfernt waren. Mithilfe von linearen Regressionsanalysen wurden Korrelationen untersucht und im Anschluss mittels des F-Tests auf signifikante Unterschiede analysiert. Die Ergebnisse sind in den Scatterplots dargestellt.

Da keine Normalverteilung der metrischen Daten angenommen werden konnte, wurde für die Untersuchung eine nichtparametrische Analyse der Faktoreinflüsse, basierend auf den Rängen der beobachteten Daten, verwendet. Für die Auswertung der Respirationsdaten wurde ein einfaktorielles Modell mit dem festen Faktor Temperatur und für die kardialen Daten ein zweifaktorielles Modell mit den zwei festen, gekreuzten Faktoren Temperatur und PCI berechnet. Für Paarvergleiche und Wechselwirkungen wurden die p-Werte mit der Tukey-Methode adjustiert. Als Signifikanzniveau wird ein Fehler 1. Art von $\alpha=5 \%$ festgesetzt. 


\section{Ergebnisse}

\subsection{Einfluss milder Hypothermie auf die respiratorische Funktion}

\subsubsection{Basisdaten}

Es wurden retrospektiv die Daten von 166 Patienten mit HKS und primär erfolgreicher Reanimation analysiert, die im Zeitraum von Januar 2003 bis Dezember 2009 im Herzzentrum der UMG mit großvolumigen kalten Infusionen therapiert wurden. Dabei wurde ein HKS außerhalb des Krankenhauses (OHCA) bei 76,51\% der Patienten dokumentiert. Das Durchschnittsalter lag bei 64,7 $\pm 1,0$ Jahren mit einer Spannweite (Spw.) von 29 bis 89 Jahren, wobei zu 75,9\% Männer und zu 24,1\% Frauen betroffen waren. Das initial abgeleitete Elektrokardiogramm (EKG) zeigte in $57,23 \%$ der Fälle ein Kammerflimmern oder eine Kammertachykardie, in 28,31\% der Fälle eine Asystolie und bei 6,63\% eine PEA. Die Hauptursache für den HKS war ein akuter Myokardinfarkt (46,99\%), gefolgt von Rhythmusereignissen bei chronisch ischämischer Herzkrankheit (18,67\%) oder anderen Kardiomyopathien $(9,04 \%)$, Hypoxie/Asphyxie (11,45\%) und Lungenembolien (4,82\%). Es zeigte sich initial eine mittelgradig eingeschränkte LV-Funktion mit einer Ejektionsfraktion (EF) von $34,8 \pm 1,1 \%$ (Spw. 10-70\%). Eine im Verlauf bei 62 Patienten erfolgte EchoKontrolle zeigte eine verbesserte EF von $41,5 \pm 1,5 \%$. auf. Die als prognostischer Parameter für das neurologische Outcome dienende neuronenspezifische Enolase (NSE) wurde bei 157 Patienten bestimmt. Während der ersten fünf Tage nach ROSC betrug sie in der Spitze 70,55 \pm 10,46 $\mu \mathrm{g} / \mathrm{l}$ (Liter) (Spw. 2,87-930,70 $\mu \mathrm{g} / \mathrm{l}$ ). Die Sterberate der behandelten Patienten innerhalb der UMG betrug 40,96\%, 98 von 166 Patienten konnten verlegt oder entlassen werden. Tabelle 3.1 stellt diese Basisdaten ausführlich dar. 


\begin{tabular}{|c|c|c|c|}
\hline Gesamtanzahl der Patienten & 166 & & \\
\hline männliches Geschlecht - Anzahl (\%) & 126 & $(75,90)$ & \\
\hline weibliches Geschlecht - Anzahl (\%) & 40 & $(24,10)$ & \\
\hline Lebensalter gesamt - Jahre (MW \pm SEM) & & & $64,7 \pm 1,0$ \\
\hline Männer - Jahre (MW \pm SEM) & & & $64,6 \pm 1,2$ \\
\hline Frauen - Jahre (MW \pm SEM) & & & $64,9 \pm 2,3$ \\
\hline \multicolumn{4}{|l|}{ Initial im EKG abgeleiteter Herzrhythmus } \\
\hline Kammerflimmern oder -tachykardie - Anzahl (\%) & 95 & $(57,23)$ & \\
\hline Asystolie - Anzahl (\%) & 47 & $(28,31)$ & \\
\hline Pulslose elektrische Aktivität - Anzahl (\%) & 11 & $(6,63)$ & \\
\hline nicht dokumentiert - Anzahl (\%) & 13 & $(7,83)$ & \\
\hline \multicolumn{4}{|l|}{ Ursache des Herzkreislaufstillstandes } \\
\hline akuter Myokardinfarkt - Anzahl (\%) & 78 & $(46,99)$ & \\
\hline $\begin{array}{l}\text { Rhythmusereignis bei chronisch ischämischer } \\
\text { Herzkrankheit - Anzahl (\%) }\end{array}$ & 31 & $(18,67)$ & \\
\hline $\begin{array}{l}\text { Rhythmusereignis bei anderen Kardiomyopathien - } \\
\text { Anzahl (\%) }\end{array}$ & 15 & $(9,04)$ & \\
\hline Hypoxie/Asphyxie - Anzahl (\%) & 19 & $(11,45)$ & \\
\hline Lungenembolie - Anzahl (\%) & 8 & $(4,82)$ & \\
\hline Andere - Anzahl (\%) & 15 & $(9,04)$ & \\
\hline prähospitaler Herzkreislaufstillstand - Anzahl (\%) & 127 & $(76,51)$ & \\
\hline lebende Entlassungen - Anzahl (\%) & 98 & $(59,04)$ & \\
\hline verstorben in der UMG - Anzahl (\%) & 68 & $(40,96)$ & \\
\hline Bestimmung NSE-Konzentration - Anzahl (\%) & 157 & $(94,58)$ & \\
\hline maximale NSE-Konzentration - $\mu \mathrm{g} / \mathrm{l}(\mathrm{MW} \pm \mathrm{SEM})$ & & & $70,55 \pm 10,46$ \\
\hline \multicolumn{4}{|l|}{ linksventrikuläre Ejektionsfraktion } \\
\hline Bestimmung im initialen Echo - Anzahl (\%) & 123 & $(74,10)$ & \\
\hline LVEF - \% (MW \pm SEM) & & & $34,8 \pm 1,1$ \\
\hline Bestimmung im letzten Echo - Anzahl (\%) & 62 & $(37,35)$ & \\
\hline LVEF - \% (MW \pm SEM) & & & $41,5 \pm 1,5$ \\
\hline
\end{tabular}

Tab. 3.1: Basisdaten des Respirations-Kollektivs. $\mathbf{n}=166$.

NSE: neuronenspezifische Enolase; LVEF: linksventrikuläre Ejektionsfraktion; Echo: Echokardiographie; EKG: Elektrokardiogramm; MW: Mittelwert; SEM: Standardfehler. 


\subsubsection{Parameter zur milden Hypothermie}

Das Zeitintervall von der Ankunft der Patienten in der UMG bis zur Ankunft auf der Intensivstation betrug durchschnittlich 111,1 \pm 5,6 Minuten. Ursachen für diese Zeitverzögerung waren in der Hauptsache mit 57,83\% Koronarangiographien, bei $41,56 \%$ erfolgte eine $\mathrm{PCl}$, und $\mathrm{CT}$-Untersuchungen (14,46\%). Bei $31,33 \%$ der Patienten wurde noch vor Ankunft auf ITS eine Echokardiographie durchgeführt.

Der Kühlungsbeginn der Patienten startete schnellstmöglich nach Ankunft in der UMG, zumeist in der Notaufnahme oder im HKL. Auch wurde die Kühlung während erfolgter Untersuchungen oder Interventionen wie PCI, CT, Echo usw. nicht unterbrochen. Daher lag die KKT bei der Ankunft auf ITS im Durchschnitt bereits bei $35,7 \pm 0,1^{\circ} \mathrm{C}$. Das Zeitintervall nach der Ankunft auf ITS bis zum Erreichen der Zieltemperatur von $\leq 34^{\circ} \mathrm{C}$ betrug $4,5 \pm 0,3$ Stunden. Dies entspricht einer durchschnittlichen Kühlungsgeschwindigkeit von $0,62 \pm 0,04^{\circ} \mathrm{C} / \mathrm{h}$. In diesem Zeitraum wurden dem Patienten durchschnittlich $3.279 \pm 151 \mathrm{ml}$ kalte Infusionen infundiert. Die durchschnittliche Zeit von der Ankunft auf ITS bis zum Erreichen einer Temperatur von $33^{\circ} \mathrm{C}$ betrug $7,8 \pm 0,5$ Stunden, was einer Kühlungsgeschwindigkeit von $0,58 \pm 0,03^{\circ} \mathrm{C} / \mathrm{h}$ entspricht. In diesem Zeitraum erhielten die Patienten $4.095 \pm$ $177 \mathrm{ml}$ an kaltem Volumen. Die genauen Kühlungsdaten können Tabelle 3.2 entnommen werden. 


Ursache der Zeitverzögerung
$\begin{array}{lll}\text { Koronarangiographie - Anzahl (\%) } & 96 & (57,83) \\ \text { PCl - Anzahl (\%) } & 69 & (41,56) \\ \text { CT - Anzahl (\%) } & 24 & (14,45) \\ \text { Echo - Anzahl (\%) } & 52 & (31,32)\end{array}$

KKT bei Ankunft ITS $-{ }^{\circ} \mathrm{C}(\mathrm{MW} \pm \mathrm{SEM})$

$35,7 \pm 0,1$

Gesamtmenge kalter Infusionen

bis $34^{\circ} \mathrm{C} \mathrm{KKT}-\mathrm{ml}(\mathrm{MW} \pm \mathrm{SEM})$

$3.279 \pm 151$

bis $33^{\circ} \mathrm{C} \mathrm{KKT} \mathrm{-} \mathrm{ml}(\mathrm{MW} \pm \mathrm{SEM})$

$4.095 \pm 177$

Zeitintervall von Ankunft ITS bis Zieltemperatur

bis $34^{\circ} \mathrm{C}-\mathrm{h}(\mathrm{MW} \pm \mathrm{SEM})$

$4,5 \pm 0,3$

bis $33^{\circ} \mathrm{C}-\mathrm{h}(\mathrm{MW} \pm \mathrm{SEM})$

$7,8 \pm 0,5$

Kühlgeschwindigkeit $\left({ }^{\circ} \mathrm{C} / \mathrm{h}\right)$

bis $34^{\circ} \mathrm{C}-{ }^{\circ} \mathrm{C} / \mathrm{h}(\mathrm{MW} \pm \mathrm{SEM})$

$0,62 \pm 0,04$

bis $33^{\circ} \mathrm{C}-{ }^{\circ} \mathrm{C} / \mathrm{h}(\mathrm{MW} \pm \mathrm{SEM})$

$0,58 \pm 0,03$

Kühlungsmethode - Induktion und Aufrechterhaltung

Einsatz kalter Infusionen - Patientenanzahl (\%)

CoolGard - Patientenanzahl (\%)

$166(100,0)$

Rest - Patientenanzahl (\%)

$49 \quad(29,52)$

$66 \quad(39,76)$

Tab. 3.2: Kühlungsparameter des Respirations-Kollektivs. $\mathbf{n}=166$.

ER: Notaufnahme; ITS: Intensivstation; PCI: perkutane koronare Intervention; Echo: Echokardiographie; CT: Computertomographie; KKT: Körperkerntemperatur; MW: Mittelwert; SEM: Standardfehler. 


\subsubsection{Parameter der respiratorischen Funktion}

Es wurde der Einfluss großvolumiger kalter Infusionen auf die respiratorische Funktion der Patienten untersucht. Bei der Ankunft auf der ITS $\left(35,7^{\circ} \mathrm{C}\right)$ betrug der $\mathrm{PaO}_{2}$ initial im Median $155,0 \mathrm{mmHg}(78,0-309 \mathrm{mmHg})$ bei einem $\mathrm{FiO}_{2}$ von $1,0(0,6-$ 1,0) und einem PEEP von 7 mbar (5-8 mbar). Der Horovitz-Quotient, als Maß für eine Schädigung der Lunge, betrug $210,5 \mathrm{mmHg}(107,0-400,0 \mathrm{mmHg})$. Die EF betrug $34,8 \pm 1,1 \%$.

\begin{tabular}{|c|c|c|c|c|c|}
\hline Ankunft ITS & Median & MIN & MAX & $25 \%-Q$ & $75 \%-Q$ \\
\hline $\mathrm{PaO}_{2}(\mathrm{mmHg})$ & 155,0 & 22,8 & 599,5 & 78,0 & 309,0 \\
\hline $\mathrm{FiO}_{2}$ & 1,00 & 0,20 & 1,00 & 0,60 & 1,00 \\
\hline PEEP (mbar) & 7,00 & 5,00 & 30,00 & 5,00 & 8,00 \\
\hline $\mathrm{PaO}_{2} / \mathrm{FiO}_{2}(\mathrm{mmHg})$ & 210,5 & 26,0 & 944,0 & 107,0 & 400,0 \\
\hline
\end{tabular}

Tab. 3.3: Beatmungsparameter und Blutgaswerte bei der Ankunft auf Intensivstation (ITS: $35,7^{\circ} \mathrm{C}$ Körperkerntemperatur). $\mathrm{n}=166$.

$\mathrm{PaO}_{2}$ : arterieller Sauerstoff-Partialdruck; PEEP: positiver endexspiratorischer Druck; $\mathrm{FiO}_{2}$ : inspiratorische Sauerstoffkonzentration; MIN: Minimum; MAX: Maximum; 25\%-Q: 25\%-Quantil; 75\%Q.: 75\%-Quantil.

Bis zum Erreichen der Zieltemperatur von $\leq 34^{\circ} \mathrm{C}$ konnte der $\mathrm{FiO}_{2}$ signifikant reduziert werden. So betrug er bei $\leq 34^{\circ} \mathrm{C}$ im Median $0,60(0,45-0,70)(p<0,0001)$, bei einem sehr leicht, aber signifikant angehobenem PEEP, der im Median jedoch weiterhin bei 7 mbar lag (5-10 mbar) $(p=0,0012)$. Der $\mathrm{PaO}_{2}$ sank signifikant auf $106,5 \mathrm{mmHg}$ $(83,4-166,0 \mathrm{mmHg})(\mathrm{p}=0,001)$, womit der $\mathrm{PaO}_{2} / \mathrm{FiO}_{2}$-Quotient trotz der Infusion von $3.279 \pm 151 \mathrm{ml}$ kalten Volumens unverändert bei $210,3 \mathrm{mmHg}(130,2-314,0 \mathrm{mmHg})$ blieb (n.s.). Die durchschnittliche Änderungsrate des $\mathrm{PaO}_{2} / \mathrm{FiO}_{2}$-Quotienten betrug im Median $-7,8 \mathrm{mmHg}(-101,7-65,2 \mathrm{mmHg})$.

\begin{tabular}{lrrrrrr}
\hline $\mathbf{3 4}^{\circ} \mathbf{C}$ & Median & MIN & MAX & $\mathbf{2 5 \% - Q}$ & $\mathbf{7 5 \% - Q}$ & p-Wert \\
\hline $\mathrm{PaO}_{2}(\mathrm{mmHg})$ & 106,5 & 12,9 & 526,0 & 83,4 & 166,0 & $=0,001$ \\
$\mathrm{FiO}_{2}$ & 0,60 & 0,26 & 1,00 & 0,45 & 0,70 & $<0,0001$ \\
$\mathrm{PEEP}_{(\mathrm{mbar})}$ & 7,00 & 3,00 & 30,00 & 5,00 & 10,00 & $=0,0012$ \\
$\mathrm{PaO}_{2} / \mathrm{FiO}_{2}(\mathrm{mmHg})$ & 210,3 & 19,8 & 841,0 & 130,2 & 314,0 & n.s. \\
\hline
\end{tabular}

Tab. 3.4: Beatmungsparameter und Blutgaswerte bei Erreichen einer Körperkerntemperatur von $34^{\circ} \mathrm{C} . \mathrm{n}=166$.

p-Wert: Signifikanzbestimmung zwischen Ankunft auf der Intensivstation und dem Erreichen von $34^{\circ} \mathrm{C}$; n.s.: nicht signifikant. $\mathrm{PaO}_{2}$ : arterieller Sauerstoff-Partialdruck; $\mathrm{FiO}_{2}$ : inspiratorische Sauerstoffkonzentration; PEEP: positiver endexspiratorischer Druck; MIN: Minimum; MAX: Maximum; 25\%-Q: 25\%-Quantil; 75\%-Q.: 75\%-Quantil. 
Nach Infusion von insgesamt $4.095 \pm 177 \mathrm{ml}$ kalten Volumens ab der Ankunft auf ITS bis zum Erreichen einer Temperatur von $33^{\circ} \mathrm{C}$ konnte der $\mathrm{FiO}_{2}$ signifikant auf im Median 0,50 $(0,40-0,65)(p<0,0001)$ reduziert werden. Der PEEP wurde sehr leicht, aber signifikant, angehoben, verblieb im Median jedoch bei 7 mbar (5-10 mbar) ( $p=$ 0,018). Der $\mathrm{PaO}_{2}$ sank auf $106,0 \mathrm{mmHg}(78,4-143,0 \mathrm{mmHg})(\mathrm{p}<0,0001)$. Der Horovitz-Quotient blieb unverändert bei 208,0 mmHg (147,0-286,0 mmHg) (n.s.). Die durchschnittliche Änderungsrate des $\mathrm{PaO}_{2} / \mathrm{FiO}_{2}$-Quotienten betrug $-21,6 \mathrm{mmHg}$ ($117,9-74,3 \mathrm{mmHg})$.

\begin{tabular}{|c|c|c|c|c|c|c|}
\hline $33^{\circ} \mathrm{C}$ & Median & MIN & MAX & $25 \%-Q$ & $75 \%-Q$ & p-Wert \\
\hline $\mathrm{PaO}_{2}(\mathrm{mmHg})$ & 106,0 & 8,3 & 255,0 & 78,4 & 143,0 & $<0,0001$ \\
\hline $\mathrm{FiO}_{2}$ & 0,50 & 0,30 & 1,00 & 0,40 & 0,65 & $<0,0001$ \\
\hline PEEP (mbar) & 7,00 & 1,00 & 28,00 & 5,00 & 10,00 & $=0,018$ \\
\hline $\mathrm{PaO}_{2} / \mathrm{FiO}_{2}(\mathrm{mmHg})$ & 208,0 & 9,2 & 545,0 & 147,0 & 286,0 & n.s. \\
\hline
\end{tabular}

Tab. 3.5: Beatmungsparameter und Blutgaswerte bei Erreichen einer Körperkerntemperatur von $33^{\circ} \mathrm{C} . \mathrm{n}=166$.

p-Wert: Signifikanzbestimmung zwischen Ankunft auf der Intensivstation und dem Erreichen von $33^{\circ} \mathrm{C}$; n.s.: nicht signifikant. $\mathrm{PaO}_{2}$ : arterieller Sauerstoff-Partialdruck; $\mathrm{FiO}_{2}$ : inspiratorische Sauerstoffkonzentration; PEEP: positiver endexspiratorischer Druck; MIN: Minimum; MAX: Maximum; 25\%-Q: 25\%-Quantil; 75\%-Q.: 75\%-Quantil.

Für die Reduktion der $\mathrm{FiO}_{2}$ bestand auch im Paarvergleich zwischen einer KKT von $34^{\circ} \mathrm{C}$ und $33^{\circ} \mathrm{C}$ eine signifikante Änderung $(p=0,0004)$, für die anderen Parameter gab es keine signifikanten Änderungen zwischen $34^{\circ} \mathrm{C}$ und $33^{\circ} \mathrm{C}$.

Eine graphische Darstellung der Daten findet sich in der Abbildung (Abb.) 3.1. Die genauen Daten zu den Boxplots können den Tabellen 3.3-3.5 entnommen werden.

Eine Tabelle mit der genauen Darstellung der einzelnen $p$-Werte findet sich im Anhang (Tab. 6.1). 

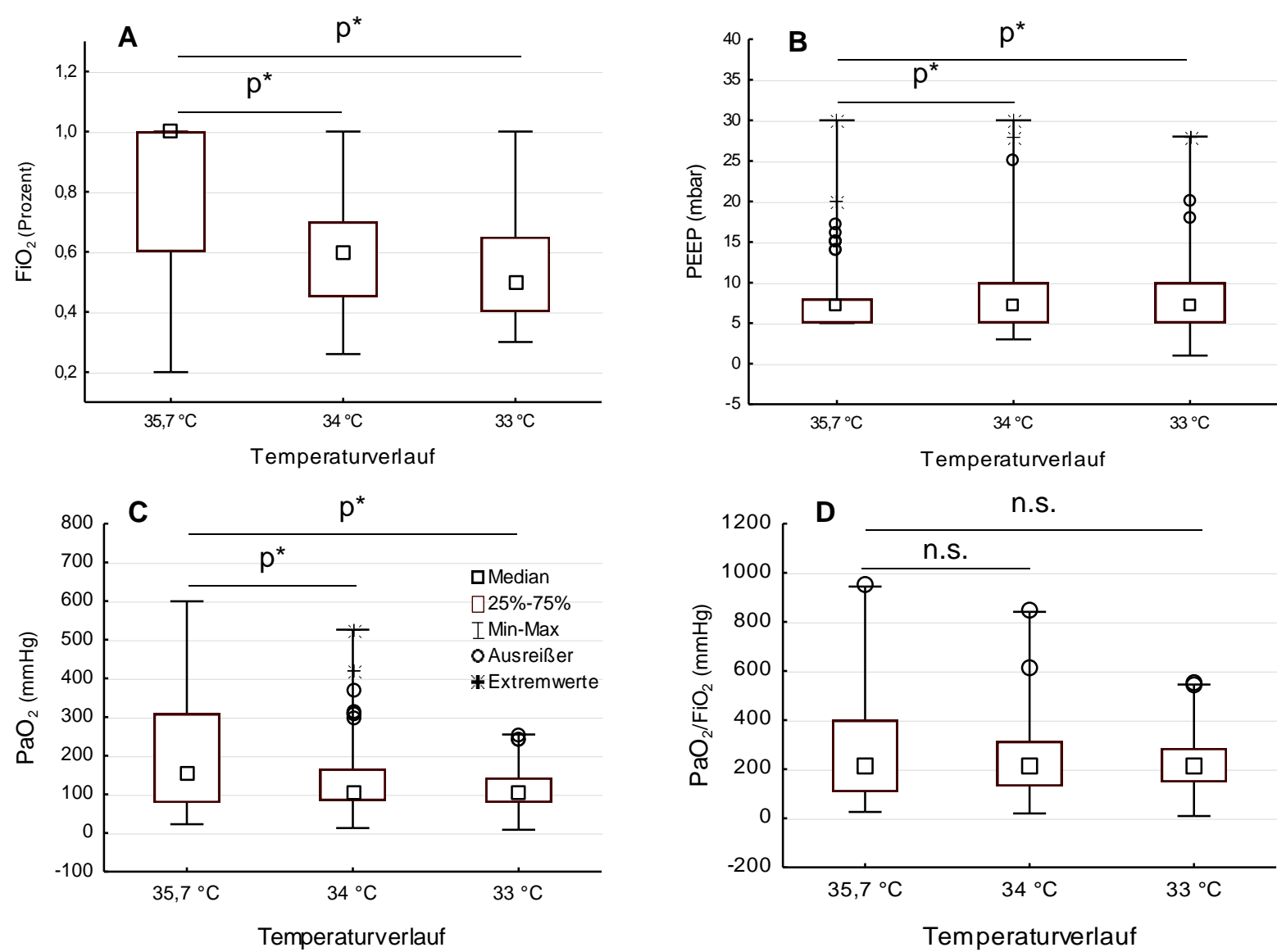

Abb. 3.1: Boxplots zum Verlauf der respiratorischen Parameter

A: inspiratorische Sauerstoffkonzentration $\left(\mathrm{FiO}_{2}\right)$; B: positiver endexspiratorischer Druck (PEEP); C: arterieller Sauerstoffpartialdruck $\left(\mathrm{PaO}_{2}\right)$; D: Horovitz-Quotient $\left(\mathrm{PaO}_{2} / \mathrm{FiO}_{2}\right)$ bei Ankunft auf der Intensivstation $\left(35,7^{\circ} \mathrm{C}\right)$, bei $34^{\circ} \mathrm{C}$ und $33^{\circ} \mathrm{C}$ Körperkerntemperatur. $\mathrm{n}=166$. $p^{*}: p<0,05$; n.s.: nicht signifikant.

Weiterhin wurde eine lineare Regressionsanalyse zwischen Horovitz-Quotient und der Menge an infundiertem kaltem Volumen durchgeführt, wie in Abbildung $3.2 \mathrm{zu}$ erkennen ist. Hier ergab sich ein schwacher, aber signifikanter, negativer linearer Zusammenhang $\left(r^{2}=0,06 ; p=0,0014\right)$ zwischen beiden Parametern bei einer infundierten Flüssigkeitsmenge zwischen 0 und $11.000 \mathrm{ml}$. 


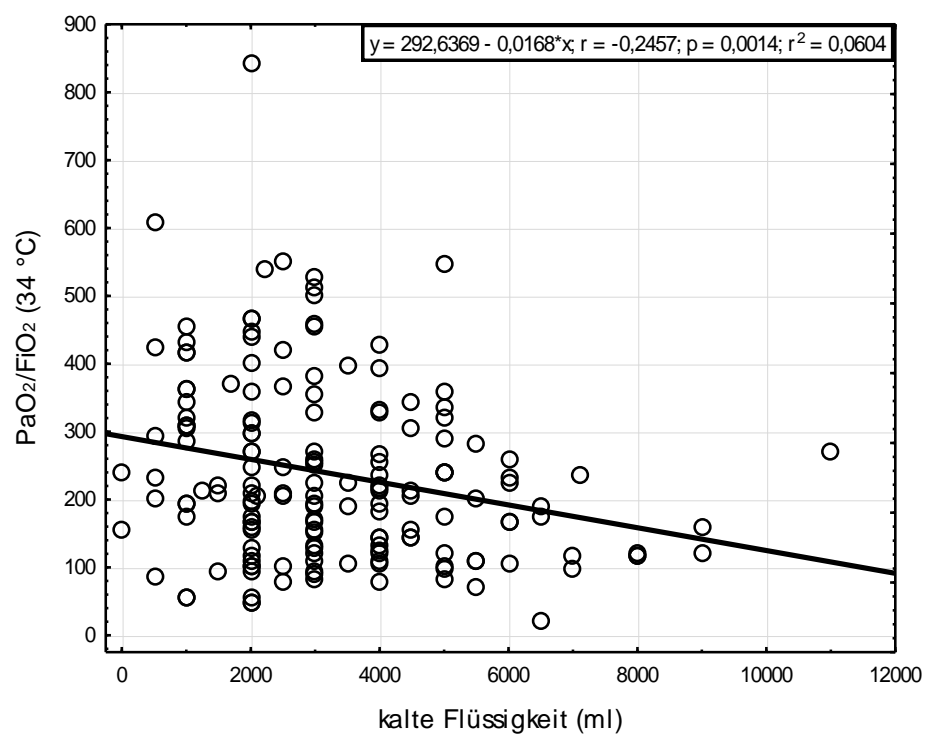

Abb. 3.2: Scatterplot zur Visualisierung der Korrelation zwischen dem Horovitz-Quotienten $\left(\mathrm{PaO}_{2} / \mathrm{FiO}_{2}\right)$ bei $34^{\circ} \mathrm{C}$ Körperkerntemperatur und der Menge des bis dahin infundierten kalten Volumens. $r^{2}=0,06, p=0,0014 ; n=166$.

Eine Regressionsanalyse bezüglich der Änderungsrate des $\mathrm{PaO}_{2} / \mathrm{FiO}_{2}$-Quotienten und der Menge an infundiertem kaltem Volumen (Abbildung 3.3) ergab keine signifikante Korrelation $\left(r^{2}=0,004 ; p=0,4241\right)$.

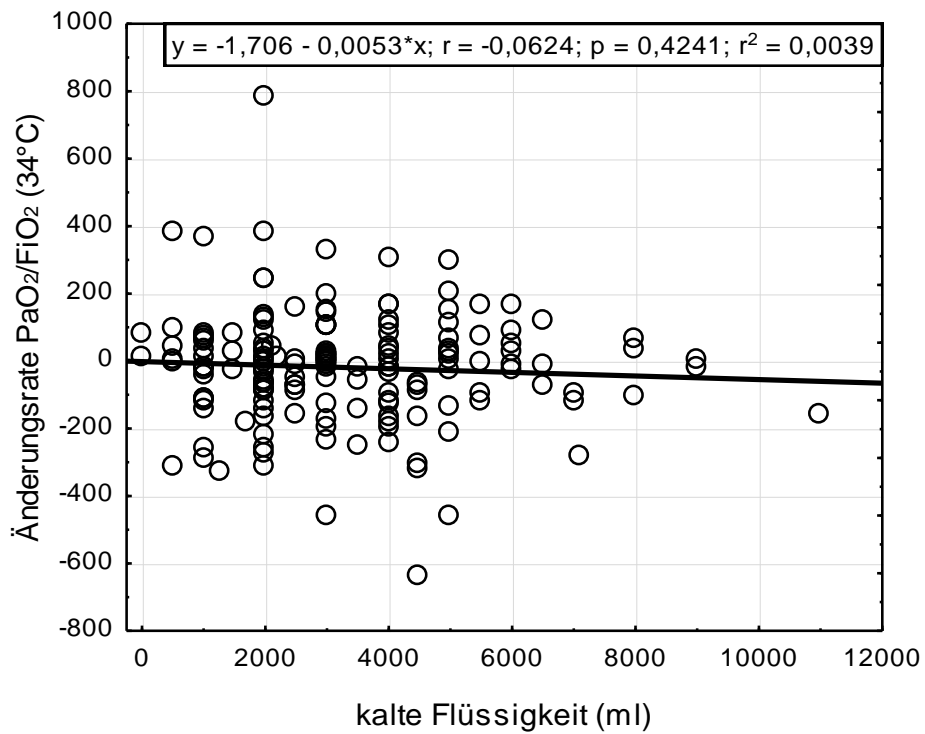

Abb. 3.3: Scatterplot zur Visualisierung der Korrelation zwischen der Änderungsrate des Horovitz-Quotienten $\left(\mathrm{PaO}_{2} / \mathrm{FiO}_{2}\right)$ bei $34^{\circ} \mathrm{C}$ Körperkerntemperatur und der Menge des bis dahin infundierten kalten Volumens. $\mathrm{r}^{2}=0,004, p=0,4241 ; n=166$. 
Bei einer Regressionsanalyse zwischen der Änderungsrate des $\mathrm{PaO}_{2} / \mathrm{FiO}_{2}-$ Quotienten und der EF konnte kein signifikanter Zusammenhang nachgewiesen werden $\left(r^{2}=0,001 ; p=0,7012 ;\right.$ Abbildung 3.4).

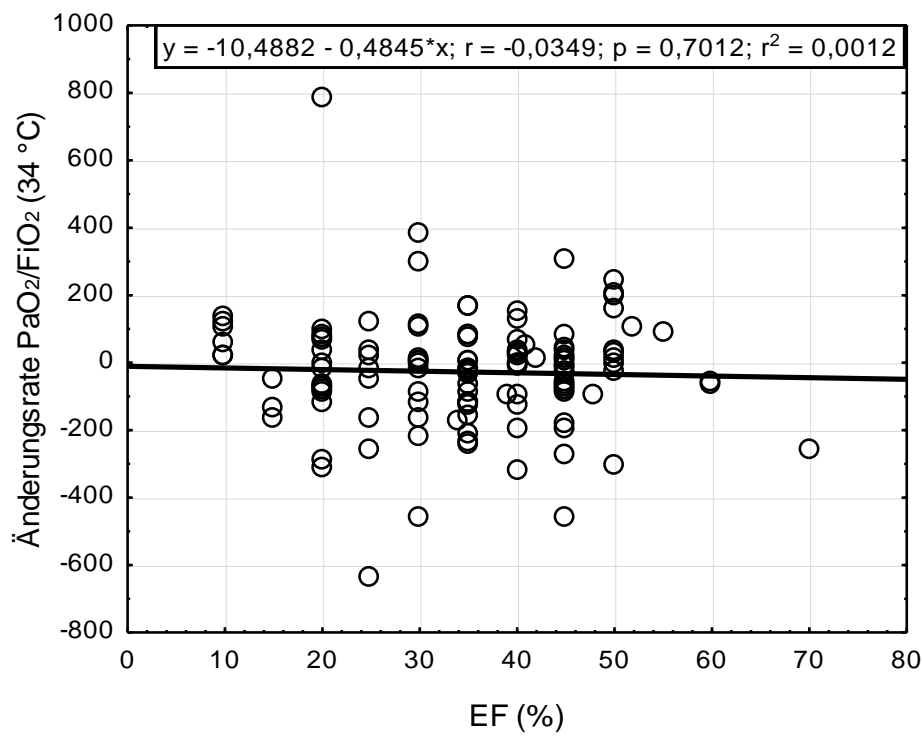

Abb. 3.4: Scatterplot zur Visualisierung der Korrelation zwischen der Änderungsrate des Horovitz-Quotienten $\left(\mathrm{PaO}_{2} / \mathrm{FiO}_{2}\right)$ bei $34^{\circ} \mathrm{C}$ Körperkerntemperatur und der Ejektionsfraktion. $\mathrm{r}^{2}=$ $0,001, p=0,7012 ; n=166$.

\subsection{Einfluss milder Hypothermie auf die Herz-Kreislauf-Funktion}

\subsubsection{Basisdaten}

Es wurden retrospektiv die Daten von 159 Patienten mit HKS und primär erfolgreicher Reanimation analysiert, die im Zeitraum von Januar 2003 bis Dezember 2009 im Herzzentrum der UMG mit milder therapeutischer Hypothermie behandelt wurden. Dabei wurde ein prähospitaler HKS bei $82,39 \%$ der Patienten dokumentiert. Das Durchschnittsalter lag bei 62,5 $\pm 1,1$ Jahren (Spw. 29 bis 86 Jahre), wobei zu 76,73\% Männer und zu 23,27\% Frauen betroffen waren. Das initial abgeleitete Elektrokardiogramm (EKG) zeigte bei 63,52\% der Fälle ein Kammerflimmern oder eine Kammertachykardie, in 24,53\% der Fälle eine Asystolie und in 6,29\% eine PEA. Die Hauptursache für den HKS war ein akuter Myokardinfarkt (47,80\%), gefolgt von Herzrhythmusstörungen bei chronisch ischämischer Herzkrankheit $(17,61 \%)$ und anderen Kardiomyopathien (11,32\%). In 11,32\% war eine Hypoxie/Asphyxie ursächlich. Seltener war die Ursache eine Lungenembolie (5,03\%). Die NSE wurde 
bei 153 Patienten bestimmt und betrug während der ersten fünf Tage nach ROSC in der Spitze 64,83 \pm 10,06 $\mu \mathrm{g} / \mathrm{l}$ (Spw. 9,3-930,7 $\mu \mathrm{g} / \mathrm{l}$ ). Die Sterberate der behandelten Patienten innerhalb der UMG betrug 30,19\%, 106 von 159 Patienten konnten verlegt oder entlassen werden. Tabelle 3.6 sind die genauen Basisdaten zu entnehmen.

\begin{tabular}{|c|c|c|c|}
\hline Gesamtanzahl der Patienten & 159 & & \\
\hline männliches Geschlecht - Anzahl (\%) & 122 & $(76,73)$ & \\
\hline weibliches Geschlecht - Anzahl (\%) & 37 & $(23,27)$ & \\
\hline Lebensalter gesamt - Jahre (MW \pm SEM) & & & $62,5 \pm 1,1$ \\
\hline Männer - Jahre (MW \pm SEM) & & & $62,6 \pm 1,1$ \\
\hline Frauen - Jahre (MW \pm SEM) & & & $62,5 \pm 2,6$ \\
\hline \multicolumn{4}{|l|}{ Initial im EKG abgeleiteter Herzrhythmus } \\
\hline Kammerflimmern oder -tachykardie - Anzahl (\%) & 101 & $(63,52)$ & \\
\hline Asystolie - Anzahl (\%) & 39 & $(24,53)$ & \\
\hline Pulslose elektrische Aktivität - Anzahl (\%) & 10 & $(6,29)$ & \\
\hline nicht dokumentiert - Anzahl (\%) & 9 & $(5,66)$ & \\
\hline \multicolumn{4}{|l|}{ Ursache des Herzkreislaufstillstandes } \\
\hline akuter Myokardinfarkt - Anzahl (\%) & 76 & $(47,80)$ & \\
\hline $\begin{array}{l}\text { Rhythmusereignis bei chronisch ischämischer } \\
\text { Herzkrankheit - Anzahl (\%) }\end{array}$ & 28 & $(17,61)$ & \\
\hline $\begin{array}{l}\text { Rhythmusereignis bei anderen Kardiomyopathien - } \\
\text { Anzahl (\%) }\end{array}$ & 18 & $(11,32)$ & \\
\hline Hypoxie/Asphyxie - Anzahl (\%) & 18 & $(11,32)$ & \\
\hline Lungenembolie - Anzahl (\%) & 8 & $(5,03)$ & \\
\hline Andere - Anzahl (\%) & 11 & $(6,92)$ & \\
\hline prähospitaler Herzkreislaufstillstand - Anzahl (\%) & 131 & $(82,39)$ & \\
\hline lebende Entlassungen - Anzahl (\%) & 106 & $(66,67)$ & \\
\hline verstorben in der UMG - Anzahl (\%) & 48 & $(30,19)$ & \\
\hline Bestimmung NSE-Konzentration - Anzahl (\%) & 153 & $(96,23)$ & \\
\hline maximale NSE-Konzentration - $\mu \mathrm{g} / \mathrm{l}(\mathrm{MW} \pm \mathrm{SEM})$ & & & $64,83 \pm 10,1$ \\
\hline
\end{tabular}

Tab. 3.6: Basisdaten des kardialen Kollektivs. $\mathbf{n}=159$.

NSE: neuronenspezifische Enolase; Echo: Echokardiographie; EKG: Elektrokardiogramm; MW: Mittelwert; SEM: Standardfehler. 


\subsubsection{Parameter zur milden Hypothermie}

Das Zeitintervall von der Ankunft des Patienten in der UMG bis zur Ankunft auf der Intensivstation betrug durchschnittlich 117,2 \pm 8,5 Minuten. Ursachen für diese Zeitverzögerung waren in 56,60\% Koronarangiographien, in 40,25\% der Fälle erfolgte eine PCl. Andere Ursachen waren CT-Untersuchungen (15,72\%) oder noch vor Ankunft auf der ITS durchgeführte Echokardiographien (36,48\%). Insgesamt wurde eine Echokardiographie bei $76,1 \%$ der Patienten schnellstmöglich nach Erreichen der UMG durchgeführt. Es zeigte sich initial eine stark verschlechterte LVEF von $34,2 \pm 1,1 \%$ (Spw. 10-70\%). Eine im Verlauf bei 75 Patienten erfolgte Echo-Kontrolle zeigte eine verbesserte EF von $40,9 \pm 1,4 \%$ auf.

Der Kühlungsbeginn des Patienten startete schnellstmöglich nach Ankunft in der UMG, also zumeist in der Notaufnahme oder im HKL. Auch wurde die Kühlung während Untersuchungen oder Interventionen ( $\mathrm{PCl}, \mathrm{CT}$, Echo usw.) nicht unterbrochen. Daher lag die Körperkerntemperatur bei der Ankunft auf ITS im Durchschnitt bereits bei $35,8 \pm 0,01^{\circ} \mathrm{C}$. Das Zeitintervall nach der Ankunft auf ITS bis zum Erreichen der Zieltemperatur von $\leq 34^{\circ} \mathrm{C}$ betrug $5,4 \pm 0,4$ Stunden. Dies entspricht einer durchschnittlichen Kühlungsgeschwindigkeit von $0,58 \pm 0,04^{\circ} \mathrm{C} / \mathrm{h}$. Die durchschnittliche Zeit von der Ankunft auf ITS bis zu einer Temperatur von $33^{\circ} \mathrm{C}$ betrug 9,1 $\pm 0,6$ Stunden, was einer Kühlungsgeschwindigkeit von $0,56 \pm 0,03^{\circ} \mathrm{C} / \mathrm{h}$ entspricht. Die Kühlungsparameter können Tabelle 3.7 entnommen werden. 


Ursache der Zeitverzögerung
$\begin{array}{lll}\text { Koronarangiographie - Anzahl (\%) } & & \\ \text { PCl - Anzahl (\%) } & 90 & (56,60) \\ \text { CT - Anzahl (\%) } & 64 & (40,25) \\ \text { Echo - Anzahl (\%) } & 25 & (15,72) \\ \end{array}$

KKT bei Ankunft ITS $-{ }^{\circ} \mathrm{C}(\mathrm{MW} \pm \mathrm{SEM})$

$35,8 \pm 0,1$

Gesamtmenge kalter Infusionen

bis $34^{\circ} \mathrm{C} \mathrm{KKT} \mathrm{-} \mathrm{ml}(\mathrm{MW} \pm \mathrm{SEM})$

$3.233 \pm 162$

bis $33^{\circ} \mathrm{C} \mathrm{KKT} \mathrm{-} \mathrm{ml}(\mathrm{MW} \pm \mathrm{SEM})$

$4.017 \pm 193$

Zeitintervall von Ankunft ITS bis Zieltemperatur

bis $34^{\circ} \mathrm{C}-\mathrm{h}(\mathrm{MW} \pm \mathrm{SEM})$

$5,4 \pm 0,4$

bis $33^{\circ} \mathrm{C}-\mathrm{h}(\mathrm{MW} \pm \mathrm{SEM})$

$9,1 \pm 0,6$

Kühlgeschwindigkeit $\left({ }^{\circ} \mathrm{C} / \mathrm{h}\right)$

bis $34^{\circ} \mathrm{C}-{ }^{\circ} \mathrm{C} / \mathrm{h}$ (MW \pm SEM)

$0,58 \pm 0,04$

bis $33^{\circ} \mathrm{C}-{ }^{\circ} \mathrm{C} / \mathrm{h}(\mathrm{MW} \pm \mathrm{SEM})$

$0,56 \pm 0,03$

Kühlungsmethode - Induktion und Aufrechterhaltung

Einsatz kalter Infusionen - Patientenanzahl (\%)

138

$(86,79)$

CoolGard - Patientenanzahl (\%)

$45 \quad(28,3)$

Rest - Patientenanzahl (\%)

$73 \quad(45,91)$

linksventrikuläre Ejektionsfraktion

Bestimmung im initialen Echo - Anzahl (\%)

121

$(76,10)$

LVEF - \% (MW \pm SEM)

$34,7 \pm 1,1$

Bestimmung im letzten Echo - Anzahl (\%)

$75 \quad(47,17)$

LVEF - \% (MW \pm SEM)

$40,9 \pm 1,4$

Tab. 3.7: Kühlungsparameter des kardialen Kollektivs. $\mathbf{n}=159$.

ER: Notaufnahme; ITS: Intensivstation; PCl: perkutane koronare Intervention; Echo: Echokardiographie; CT: Computertomographie; KKT: Körperkerntemperatur; LVEF: linksventrikuläre Ejektionsfraktion; MW: Mittelwert; SEM: Standardfehler. 


\subsubsection{Parameter der Herz-Kreislauf-Funktion}

\subsubsection{Einfluss auf die Katecholamin-Dosierung}

Es wurde der Einfluss der MTH auf die Katecholamin-Dosierung sowie auf die Hämodynamik der Patienten untersucht. 64 von 159 Patienten $(40,25 \%)$ erhielten vor Erreichen der ITS eine PCI. Da eine Wiedereröffnung der Koronarien einen Einfluss auf die Erholung der Kontraktionskraft des Herzens haben kann, wurden die Daten im Hinblick auf unterschiedliche Katecholamindosen, je nach Temperaturwert und Vorliegen einer $\mathrm{PCI}$, untersucht.

In der statistischen Analyse zeigte sich bei der Höhe der Noradrenalin-Dosierung eine signifikante Wechselwirkung zwischen Temperaturänderung und $\mathrm{PCl}$ (erhalten oder nicht erhalten) $(p=0,0031)$.

Noradrenalin: Aus diesem Grunde erfolgte für die Analyse der NoradrenalinDosierung eine Aufteilung des Gesamtkollektivs (159 Patienten) in die beiden Kollektive „PCl nicht erhalten“ ( $\mathrm{PCl}=0$ mit 95 Patienten) und „PCl erhalten“ $(\mathrm{PCl}=1$ mit 64 Patienten).

$\mathbf{P C I}=0$ : Bei der Ankunft auf ITS wurden 32/95 Patienten mit NA versorgt. Aufgrund dieser geringen Behandlungszahl betrug die NA-Gabe im Median 0,0 $\mu \mathrm{g} / \mathrm{min}$ (MW 3,7 $\mu \mathrm{g} / \mathrm{min}$; Spw. 0,0-40,0 $\mu \mathrm{g} / \mathrm{min})$. Während der Kühlungsinduktion stieg die NADosierung signifikant an. So betrug sie bei $34^{\circ} \mathrm{C}$ im Median 2,0 $\mu \mathrm{g} / \mathrm{min}$ (MW 4,9 $\mu \mathrm{g} / \mathrm{min}$; Spw. 0,0-52,0 $\mu \mathrm{g} / \mathrm{min}$ ) bei einem behandelten Anteil von 52/95 Patienten ( $\mathrm{p}=$ $0,0237)$ und bei $33^{\circ} \mathrm{C}(56 / 95$ Patienten) ebenfalls $2,0 \mu \mathrm{g} / \mathrm{min}$ (MW 4,3 $\mu \mathrm{g} / \mathrm{min}$; Spw. 0,0-41,0 $\mu \mathrm{g} / \mathrm{min} ; \mathrm{p}=0,0132$ gegen Ankunft ITS). Bis zum Ende der 24-stündigen Kühlungsphase bei $33^{\circ} \mathrm{C}(71 / 95$ Patienten) stieg die NA-Dosierung signifikant auf 4,0 $\mu \mathrm{g} / \mathrm{min} \quad$ (MW 6,1 $\mu \mathrm{g} / \mathrm{min} ;$ Spw. 0,0-41,0 $\mu \mathrm{g} / \mathrm{min} ; \quad \mathrm{p}<0,0001)$ an, nach Wiedererwärmung auf $36,5^{\circ} \mathrm{C}(71 / 95$ Patienten) betrug sie im Median 6,0 $\mu \mathrm{g} / \mathrm{min}$ (MW 10,1 $\mu \mathrm{g} / \mathrm{min}$; Spw. 0,0-72,0 $\mu \mathrm{g} / \mathrm{min}$; p<0,0001) (beide Werte im Vergleich zur Ankunft auf ITS). Aus Tabelle 6.3 (Anhang) ist ersichtlich, dass auch ein signifikanter Unterschied in der NA-Dosierung zu den weiteren verschiedenen Temperaturpunkten untereinander besteht, abgesehen von einem Unterschied zwischen $34^{\circ} \mathrm{C}$ und $33^{\circ} \mathrm{C}$. 


\begin{tabular}{lrrrrrrr}
\hline Noradrenalin $(\boldsymbol{\mu g} / \mathbf{m i n})$ & Median & MW & MIN & MAX & $\mathbf{2 5 \% - Q}$ & $\mathbf{7 5 \% - Q}$ & Ant. \\
\hline Ankunft ITS & 0,0 & 3,7 & 0,0 & 40,0 & 0,0 & 4,0 & $32 / 95$ \\
$34^{\circ} \mathrm{C}$ & 2,0 & 4,9 & 0,0 & 52,0 & 0,0 & 7,0 & $52 / 95$ \\
$33^{\circ} \mathrm{C}$ & 2,0 & 4,3 & 0,0 & 41,0 & 0,0 & 6,0 & $56 / 95$ \\
vor Wiedererwärmung & 4,0 & 6,1 & 0,0 & 41,0 & 0,0 & 8,0 & $71 / 95$ \\
$36,5^{\circ} \mathrm{C}$ & 6,0 & 10,1 & 0,0 & 72,0 & 0,0 & 13,0 & $71 / 95$ \\
\hline
\end{tabular}

Tab. 3.8: Kennzahlen zur Noradrenalin-Dosierung innerhalb der einzelnen Temperaturpunkte Ankunft auf der Intensivstation (ITS: $\left.35,8^{\circ} \mathrm{C}\right), 34^{\circ} \mathrm{C}, 33^{\circ} \mathrm{C}$, vor Wiedererwärmung und $36,5^{\circ} \mathrm{C}$ Körperkerntemperatur des Subkollektivs $\mathrm{PCl}=0$ (keine perkutane koronare Intervention erhalten). $\mathbf{n}=95$.

MW: Mittelwert; MIN: Minimum; MAX: Maximum; 25\%-Q: 25\%-Quantil; 75\%-Q: 75\%-Quantil; Ant.: Anteil der mit Noradrenalin behandelten Patienten von der Gesamtzahl der Patienten.

PCI = 1: In diesem Kollektiv wurden bei der Ankunft auf ITS 20/64 Patienten mit NA therapiert. Aufgrund der geringen Zahl wurden auch hier im Median 0,0 $\mu \mathrm{g} / \mathrm{min}$ verabreicht (MW 4,6 $\mu \mathrm{g} / \mathrm{min}$; Spw. 0,0-80,0 $\mu \mathrm{g} / \mathrm{min}$ ). Während der Kühlungsphase stieg die NA-Dosierung signifikant an. Bei $34^{\circ} \mathrm{C}$ (43/64 Patienten) betrug die Infusionsrate im Median 3,5 $\mu \mathrm{g} / \mathrm{min}$ (MW 6,8 $\mu \mathrm{g} / \mathrm{min}$; Spw. 0,0-40,0 $\mu \mathrm{g} / \mathrm{min}$; $\mathrm{p}<0,0001$ ) und bei $33^{\circ} \mathrm{C}$ (46/64 Patienten) 4,0 $\mu \mathrm{g} / \mathrm{min}$ (MW 8,2 $\mu \mathrm{g} / \mathrm{min}$; Spw. 0,0-60,0 $\mu \mathrm{g} / \mathrm{min} ; \quad p<0,0001$ gegen Ankunft ITS). Bis zum Ende der 24-stündigen Kühlungsphase bei $33^{\circ} \mathrm{C}(52 / 64$ Patienten) stieg die NA-Therapie signifikant auf 6,5 $\mu \mathrm{g} / \mathrm{min} \quad$ (MW 11,0 $\mu \mathrm{g} / \mathrm{min}$; Spw. 0,0-128,0 $\mu \mathrm{g} / \mathrm{min} ; \quad \mathrm{p}<0,0001$ ) an, nach Wiedererwärmung auf $36,5^{\circ} \mathrm{C}$ (48/64 Patienten) betrug sie im Median $5,5 \mu \mathrm{g} / \mathrm{min}$ (MW 8,9 $\mu \mathrm{g} / \mathrm{min}$; Spw. 0,0-84,0 $\mu \mathrm{g} / \mathrm{min} ; \mathrm{p}<0,0001$ ) (beide Werte im Vergleich zur Ankunft ITS). Aus Tabelle 6.4 (Anhang) geht hervor, dass im weiteren Paarvergleich untereinander kein signifikanter Unterschied in der NA-Dosierung zu den verschiedenen Temperaturpunkten besteht, abgesehen von einem signifikanten Unterschied zwischen $34^{\circ} \mathrm{C}$ und dem Ende der Kühlungsphase.

\begin{tabular}{lrrrrrrr}
\hline Noradrenalin $(\boldsymbol{\mu g} / \mathbf{m i n})$ & Median & MW & MIN & MAX & $\mathbf{2 5 \% - Q}$ & $\mathbf{7 5 \% - Q}$ & Ant. \\
\hline Ankunft ITS & 0,0 & 4,6 & 0,0 & 80,0 & 0,0 & 5,0 & $20 / 64$ \\
$34^{\circ} \mathrm{C}$ & 3,5 & 6,8 & 0,0 & 40,0 & 0,0 & 10,0 & $43 / 64$ \\
$33^{\circ} \mathrm{C}$ & 4,0 & 8,2 & 0,0 & 60,0 & 0,0 & 11,0 & $46 / 64$ \\
vor Wiedererwärmung & 6,5 & 11,0 & 0,0 & 128,0 & 2,0 & 14,0 & $52 / 64$ \\
$36,5^{\circ} \mathrm{C}$ & 5,5 & 8,9 & 0,0 & 84,0 & 0,5 & 11,5 & $48 / 64$ \\
\hline
\end{tabular}

Tab. 3.9: Kennzahlen zur Noradrenalin-Dosierung innerhalb der einzelnen Temperaturpunkte Ankunft auf der Intensivstation (ITS: $\left.35,8^{\circ} \mathrm{C}\right), 34^{\circ} \mathrm{C}, 33^{\circ} \mathrm{C}$, vor Wiedererwärmung und $36,5^{\circ} \mathrm{C}$ Körperkerntemperatur des Subkollektivs $\mathbf{P C I}=1$ (perkutane koronare Intervention erhalten). $\mathbf{n}=$ 64.

MW: Mittelwert; MIN: Minimum; MAX: Maximum; 25\%-Q: 25\%-Quantil; 75\%-Q: 75\%-Quantil; Ant.: Anteil der mit Noradrenalin behandelten Patienten von der Gesamtzahl der Patienten. 
Die Boxplots der Abbildung 3.5 veranschaulichen, dass Patienten mit erhaltener PCl im Vergleich zu dem Kollektiv, welches keine PCl erhielt, eine signifikant höhere Noradrenalin-Dosierung unter Einfluss der Temperaturänderung benötigen ( $p=$ 0,0031). Genaue Daten der Boxplots können den Tabellen 3.8 und 3.9 entnommen werden.

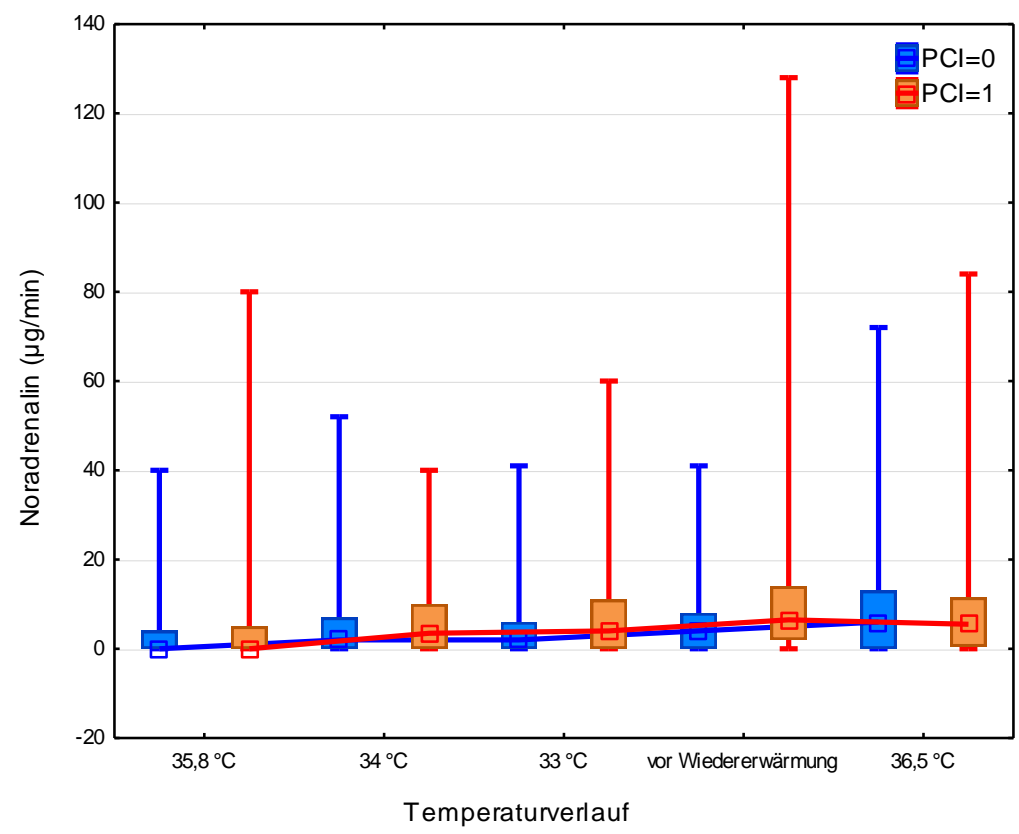

Abb. 3.5: Boxplots zum Verlauf der Noradrenalin-Dosierung bei Ankunft auf der Intensivstation $\left(35,8^{\circ} \mathrm{C}\right)$, bei $34^{\circ} \mathrm{C}, 33^{\circ} \mathrm{C}$, vor Wiedererwärmung und $36,5^{\circ} \mathrm{C}$ Körperkerntemperatur. Aufspaltung in $\mathrm{PCl}=0$ (keine perkutane koronare Intervention erhalten; $n=95)$ und $\mathrm{PCl}=1(\mathrm{PCl}$ erhalten; $\mathbf{n}=$ 64).

Adrenalin: Auch in der Adrenalin-Dosierung gab es zu den verschiedenen Temperaturpunkten signifikante Unterschiede, allerdings keine Wechselwirkung zu einer $\mathrm{PCl}$, weshalb die Kollektive nicht separat betrachtet wurden. Bei der Ankunft auf ITS erhielten 64/159 Patienten Adrenalin (Median 0,0 $\mu \mathrm{g} / \mathrm{min}$; MW 5,9 $\mu \mathrm{g} / \mathrm{min}$; Spw. $0,0-40,0 \mu \mathrm{g} / \mathrm{min})$. Während des Herunterkühlens auf $\leq 34^{\circ} \mathrm{C}(p<0,0001)$ und $33^{\circ} \mathrm{C}(p<0,0001$ gegen Ankunft ITS) konnte die Infusionsrate von Adrenalin signifikant gesenkt werden, im Median betrug sie dennoch weiterhin $0,0 \mu \mathrm{g} / \mathrm{min}$. Bei $\leq 34^{\circ} \mathrm{C}$ betrug der MW 2,8 $\mu \mathrm{g} / \mathrm{min}$ und die Spw. 0,0-100,0, $\mu \mathrm{g} / \mathrm{min}$ (41/159 Patienten), bei $33^{\circ} \mathrm{C}$ wurden im Mittel $1,4 \mu \mathrm{g} / \mathrm{min}$ Adrenalin eingesetzt mit einer Spannweite von 0,0-20,0 $\mu \mathrm{g} / \mathrm{min}$ (33/159 Patienten). Bis zum Ende der 24-stündigen Kühlungsphase bei $33^{\circ} \mathrm{C}\left(29 / 159\right.$ Patienten) und nach anschließender Wiedererwärmung auf $36,5^{\circ} \mathrm{C}$ (27/159 Patienten) sank die Adrenalin-Therapie signifikant ( $p<0,0001$ für beide 
Werte) im Vergleich zur Ankunft auf ITS; aufgrund der geringen Behandlungszahl verblieb sie aber im Median bei 0,0 $\mu \mathrm{g} / \mathrm{min}$ (Kühlungsphase: MW 1,5 $\mu \mathrm{g} / \mathrm{min}$, Spw. 0,0-20,0 $\mu \mathrm{g} / \mathrm{min} ; 36,5^{\circ} \mathrm{C}: \mathrm{MW} 1,7 \mu \mathrm{g} / \mathrm{min}$, Spw. 0,0-60,0 $\left.\mu \mathrm{g} / \mathrm{min}\right)$.

Tabelle 6.5 (Anhang) stellt dar, dass keine weiteren signifikanten Paarvergleiche aufgedeckt werden konnten. Eine graphische Aufbereitung der Daten findet sich in der Abbildung 3.6; Tabelle 3.10 stellt die zugehörigen Boxplot-Daten dar.

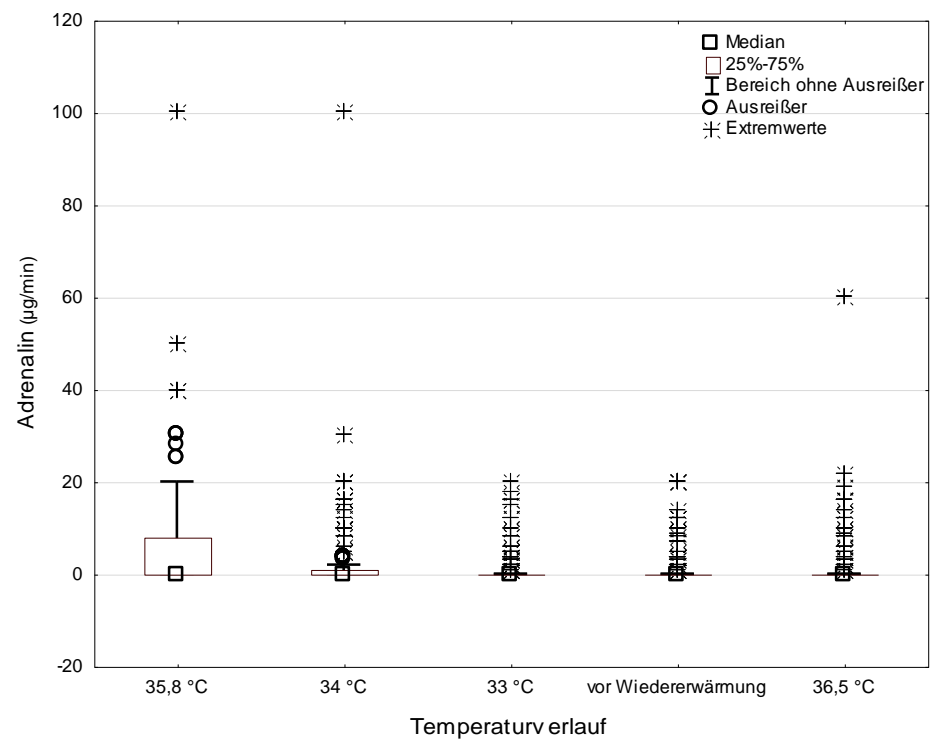

Abb. 3.6: Boxplots zum Verlauf der Adrenalin-Dosierung bei Ankunft auf der Intensivstation $\left(35,8^{\circ} \mathrm{C}\right), 34^{\circ} \mathrm{C}, 33^{\circ} \mathrm{C}$, vor Wiedererwärmung und $36,5^{\circ} \mathrm{C}$ Körperkerntemperatur. $\mathrm{n}=159$.

\begin{tabular}{lrrrrrrr}
\hline Adrenalin $(\boldsymbol{\mu g} / \mathbf{m i n})$ & Median & MW & MIN & MAX & $\mathbf{2 5 \% - Q}$ & $\mathbf{7 5 \% - Q}$ & Ant. \\
\hline Ankunft ITS & 0,0 & 5,9 & 0,0 & 100,0 & 0,0 & 8,0 & $64 / 159$ \\
$34^{\circ} \mathrm{C}$ & 0,0 & 2,8 & 0,0 & 100,0 & 0,0 & 1,0 & $41 / 159$ \\
$33^{\circ} \mathrm{C}$ & 0,0 & 1,4 & 0,0 & 20,0 & 0,0 & 0,0 & $33 / 159$ \\
vor Wiedererwärmung & 0,0 & 1,5 & 0,0 & 20,0 & 0,0 & 0,0 & $29 / 159$ \\
$36,5^{\circ} \mathrm{C}$ & 0,0 & 1,7 & 0,0 & 60,0 & 0,0 & 0,0 & $27 / 159$ \\
\hline
\end{tabular}

Tab. 3.10: Kennzahlen zur Adrenalin-Dosierung innerhalb der einzelnen Temperaturpunkte Ankunft auf der Intensivstation (ITS: $\left.35,8^{\circ} \mathrm{C}\right), 34^{\circ} \mathrm{C}, 33^{\circ} \mathrm{C}$, vor Wiedererwärmung und $36,5^{\circ} \mathrm{C}$ Körperkerntemperatur. $\mathrm{n}=159$.

MW: Mittelwert; MIN: Minimum; MAX: Maximum; 25\%-Q: 25\%-Quantil; 75\%-Q: 75\%-Quantil. Ant.: Anteil der mit Adrenalin behandelten Patienten von der Gesamtzahl der Patienten. 
Dobutamin: Die Gabe von Dobutamin zeigte in der Analyse keine signifikanten Unterschiede zu den Temperaturpunkten oder in der Wechselwirkung mit Temperatur und $\mathrm{PCl}$ auf. Die Infusionsrate betrug zu allen Zeitpunkten im Median 0,0 $\mathrm{ml} / \mathrm{h}$. Es erhielten 10/159 Patienten bei der Ankunft auf der ITS Dobutamin (MW 0,4 $\mathrm{ml} / \mathrm{h}$; Spw. 0,0-20,0 ml/h). Im Kühlungsverlauf veränderte sich die Infusionsrate von Dobutamin leicht (n.s.) auf im Mittel $0,3 \mathrm{ml} / \mathrm{h}$ bei $34^{\circ} \mathrm{C}$ (Spw. 0,0-8,0 ml/h; 14/159 Patienten) und $0,5 \mathrm{ml} / \mathrm{h}$ bei $33^{\circ} \mathrm{C}$ (Spw. $0,0-12,0 \mathrm{ml} / \mathrm{h} ; 17 / 159$ Patienten). Bis zum Ende der 24-stündigen Kühlungsphase bei $33^{\circ} \mathrm{C}$ erhielten 20/159 Patienten Dobutamin (MW 0,5 ml/h; Spw. 0,0-13,0 ml/h) und, nach Wiedererwärmung auf $36,5^{\circ} \mathrm{C}$ 14/159 Patienten (MW 0,3 ml/h; Spw. 0,0-10,0 ml/h) (Abbildung 3.7 und Tabelle 3.11).

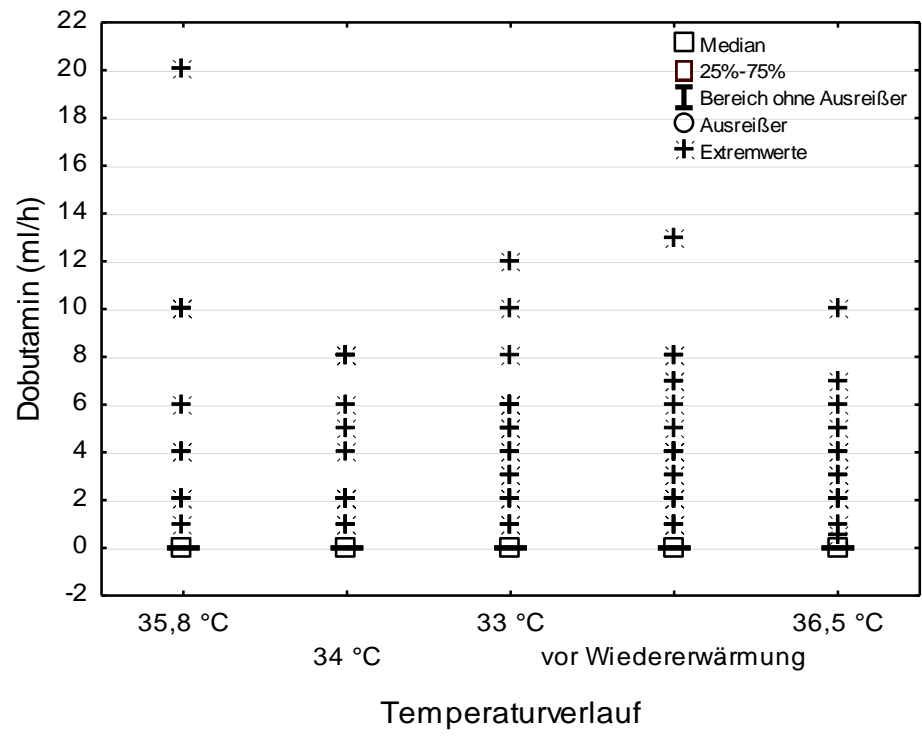

Abb. 3.7: Boxplots zum Verlauf der Dobutamin-Dosierung bei Ankunft auf der Intensivstation $\left(35,8^{\circ} \mathrm{C}\right), 34^{\circ} \mathrm{C}, 33^{\circ} \mathrm{C}$, vor Wiedererwärmung und $36,5^{\circ} \mathrm{C}$ Körperkerntemperatur. $\mathrm{n}=159$.

\begin{tabular}{lrrrrrrr}
\hline Dobutamin (ml/h) & Median & MW & MIN & MAX & 25\%-Q & 75\%-Q & Ant. \\
\hline Ankunft ITS & 0,0 & 0,4 & 0,0 & 20,0 & 0,0 & 0,0 & $10 / 159$ \\
$34^{\circ} \mathrm{C}$ & 0,0 & 0,3 & 0,0 & 8,0 & 0,0 & 0,0 & $14 / 159$ \\
$33^{\circ} \mathrm{C}$ & 0,0 & 0,5 & 0,0 & 12,0 & 0,0 & 0,0 & $17 / 159$ \\
vor Wiedererwärmung & 0,0 & 0,5 & 0,0 & 13,0 & 0,0 & 0,0 & $20 / 159$ \\
$36,5^{\circ} \mathrm{C}$ & 0,0 & 0,3 & 0,0 & 10,0 & 0,0 & 0,0 & $14 / 159$ \\
\hline
\end{tabular}

Tab. 3.11: Kennzahlen zur Dobutamin-Dosierung innerhalb der einzelnen Temperaturpunkte Ankunft auf der Intensivstation (ITS: $\left.35,8^{\circ} \mathrm{C}\right), 34^{\circ} \mathrm{C}, 33^{\circ} \mathrm{C}$, vor Wiedererwärmung und $36,5^{\circ} \mathrm{C}$ Körperkerntemperatur. $\mathrm{n}=159$.

MW: Mittelwert; MIN: Minimum; MAX: Maximum; 25\%-Q: 25\%-Quantil; 75\%-Q: 75\%-Quantil; Ant.: Anteil der mit Dobutamin behandelten Patienten von der Gesamtzahl der Patienten. 


\subsubsection{Einfluss auf Blutdruck und Herzfrequenz}

Sowohl der mittlere arterielle Blutdruck (MAP) als auch die Herzfrequenz (HF) veränderten sich zu den definierten Temperaturpunkten signifikant. Bei der Ankunft auf ITS lag der MAP im Median bei 83,3 mmHg (MW 85,6 mmHg; Spw. 30,0-173,3 $\mathrm{mmHg}$ ) und die HF bei 90/min (MW 90,3/min; Spw. 25-160/min). Während der Kühlungsphase sank der MAP signifikant auf im Median 76,7 mmHg (MW 79,4 $\mathrm{mmHg}$; Spw. $46,7-140,0 \mathrm{mmHg})$ bei $34^{\circ} \mathrm{C}(\mathrm{p}=0,0203)$ sowie $75,0 \mathrm{mmHg}$ (MW 77,0 $\mathrm{mmHg}$; Spw. $45,0-113,3 \mathrm{mmHg})$ bei $33^{\circ} \mathrm{C}(\mathrm{p}=0,0007$ gegen Ankunft auf ITS). Die HF reduzierte sich gleichzeitig auf 70/min (MW 72,8/min; Spw. 35-125/min) bei $34^{\circ} \mathrm{C}$ $(p<0,0001)$ sowie $65 / \mathrm{min}$ (MW 67,6/min; Spw. 40-115/min) bei $33^{\circ} \mathrm{C}(p<0,0001$ gegen Ankunft auf ITS). Am Ende der 24-stündigen Kühlungsphase bei $33^{\circ} \mathrm{C}$ betrug der MAP 73,3 mmHg (MW 73,8 mmHg; Spw. 38,3-110,0 mmHg; $p<0,0001$ gegen Ankunft auf ITS) und die HF lag bei 65/min (MW 67,8/min; Spw. 40-115/min; $p<0,0001$ gegen Ankunft auf ITS). Nach Wiedererwärmung auf $36,5^{\circ} \mathrm{C}$ betrug der MAP stabil 73,3 mmHg (MW 74,1 mmHg; Spw. 46,7-103,3 mmHg; $p<0,0001$ gegen Ankunft auf ITS) und die HF lag bei 90/min (MW 89/min; Spw. 50-155/min; n.s gegen Ankunft auf ITS).

In den Tabellen 6.6 und 6.7 (Anhang) ist zu erkennen, dass zudem im weiteren Paarvergleich untereinander signifikante Unterschiede in der Höhe des MAP sowie in der Höhe der HF zu den verschiedenen Temperaturpunkten bestehen. So ist die Reduktion des MAP zwischen $34^{\circ} \mathrm{C}$ und dem Ende der 24-stündigen Kühlungsphase ebenfalls signifikant $(p=0,0003)$. Zwischen $34^{\circ} \mathrm{C}$ und $33^{\circ} \mathrm{C}$ bestehen jedoch keine signifikanten Unterscheide in der Höhe des MAP. Gleiches gilt für die Beeinflussung der HF durch die Temperatur.

Eine graphische Aufbereitung findet sich in den Abbildungen 3.8 und 3.9, die dazugehörigen Daten in den Tabellen 3.12 und 3.13. 


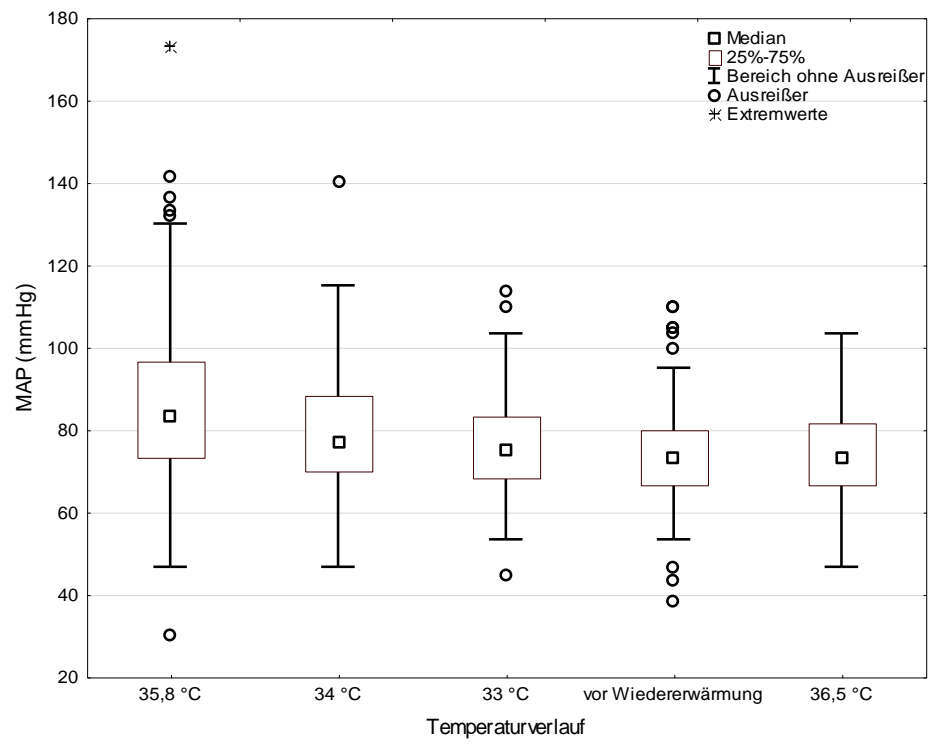

Abb. 3.8: Boxplots zum Verlauf des mittleren arteriellen Blutdrucks (MAP) bei Ankunft auf der Intensivstation $\left(35,8^{\circ} \mathrm{C}\right), 34^{\circ} \mathrm{C}, 33^{\circ} \mathrm{C}$, vor Wiedererwärmung und $36,5^{\circ} \mathrm{C}$ Körperkerntemperatur. $\mathrm{n}=$ 159.

\begin{tabular}{lrrrrrr}
\hline MAP (mmHg) & Median & MW & MIN & MAX & 25\%-Q & $\mathbf{7 5 \% - Q}$ \\
\hline Ankunft ITS & 83,3 & 85,6 & 30,0 & 173,3 & 73,3 & 96,7 \\
$34^{\circ} \mathrm{C}$ & 76,7 & 79,4 & 46,7 & 140,0 & 70,0 & 88,3 \\
$33^{\circ} \mathrm{C}$ & 75,0 & 77,0 & 45,0 & 113,3 & 68,3 & 83,3 \\
vor Wiedererwärmung & 73,3 & 73,8 & 38,3 & 110,0 & 66,7 & 80,0 \\
$36,5^{\circ} \mathrm{C}$ & 73,3 & 74,1 & 46,7 & 103,3 & 66,7 & 81,7 \\
\hline
\end{tabular}

Tab. 3.12: Kennzahlen zum mittleren arteriellen Blutdruck (MAP) innerhalb der einzelnen Temperaturpunkte Ankunft auf der Intensivstation (ITS: $35,8^{\circ} \mathrm{C}$ ), $34^{\circ} \mathrm{C}, 33^{\circ} \mathrm{C}$, vor Wiedererwärmung und 36,5 $5^{\circ} \mathrm{C}$ Körperkerntemperatur. $\mathrm{n}=159$.

MW: Mittelwert; MIN: Minimum; MAX: Maximum; 25\%-Q: 25\%-Quantil; 75\%-Q: 75\%-Quantil. 


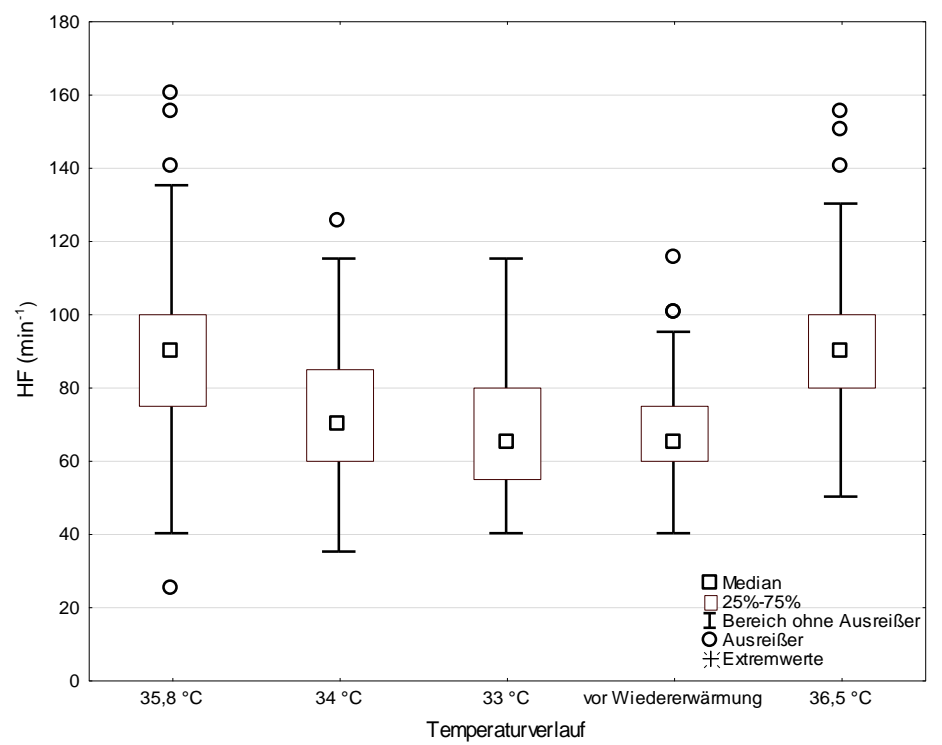

Abb. 3.9: Boxplots zum Verlauf der Herzfrequenz (HF) bei Ankunft auf der Intensivstation $\left(35,8^{\circ} \mathrm{C}\right)$, $34^{\circ} \mathrm{C}, 33^{\circ} \mathrm{C}$, vor Wiedererwärmung und $36,5^{\circ} \mathrm{C}$ Körperkerntemperatur. $\mathrm{n}=159$.

\begin{tabular}{lrrrrrr}
\hline HF $\left(\mathbf{m i n}^{-1}\right)$ & Median & MW & MIN & MAX & 25\%-Q & 75\%-Q \\
\hline Ankunft ITS & 90,0 & 90,3 & 25,0 & 160,0 & 75,0 & 100,0 \\
$34^{\circ} \mathrm{C}$ & 70,0 & 72,8 & 35,0 & 125,0 & 60,0 & 85,0 \\
$33^{\circ} \mathrm{C}$ & 65,0 & 67,6 & 40,0 & 115,0 & 55,0 & 80,0 \\
vor Wiedererwärmung & 65,0 & 67,8 & 40,0 & 115,0 & 60,0 & 75,0 \\
$36,5^{\circ} \mathrm{C}$ & 90,0 & 89,0 & 50,0 & 155,0 & 80,0 & 100,0 \\
\hline
\end{tabular}

Tab. 3.13: Kennzahlen zur Herzfrequenz (HF) innerhalb der einzelnen Temperaturpunkte Ankunft auf der Intensivstation (ITS: $\left.35,8^{\circ} \mathrm{C}\right), 34^{\circ} \mathrm{C}, 33^{\circ} \mathrm{C}$, vor Wiedererwärmung und $36,5^{\circ} \mathrm{C}$ Körperkerntemperatur. $\mathrm{n}=159$.

MW: Mittelwert; MIN: Minimum; MAX: Maximum; 25\%-Q: 25\%-Quantil; 75\%-Q: 75\%-Quantil.

\subsubsection{Einfluss des pH-Wertes auf die Katecholamin-Dosierung}

Da die Wirksamkeit von Katecholaminen durch den arteriellen pH-Wert beeinflusst werden kann, wurde der pH-Wert während der Hypothermie-Phase bestimmt. Es ergab sich ein signifikanter Unterschied bezüglich der einzelnen Temperaturpunkte. Bei Erreichen der ITS hatten die Patienten im Median einen arteriellen pH-Wert von 7,33 (MW 7,33; Spw. 7,0-7,64), was einer milden Azidose entspricht. Im Verlauf der Kühlungsperiode normalisierte sich der arterielle $\mathrm{pH}$. Bei einer Temperatur von $34^{\circ} \mathrm{C}$ (und ebenso bei $33^{\circ} \mathrm{C}$ ) lag er im Median wieder bei 7,42 (34 ${ }^{\circ} \mathrm{C}$ : MW 7,41; Spw. 7,147,66; 33ㄷ: MW 7,42; Spw. 7,16-7,71; p<0,0001), am Ende der 24-stündigen Kühlungsphase bei 7,41 (MW 7,40; Spw. 7,13-7,56; $p<0,0001$; alle Werte im Vergleich zu der Ankunft auf ITS). Bei einer Temperatur von $36,5^{\circ} \mathrm{C}$ sank er wieder 
auf 7,36 (MW 7,36; Spw. 7,10-7,53), was im Vergleich zu einer Temperatur von $34^{\circ} \mathrm{C}, 33^{\circ} \mathrm{C}$ und dem Kühlungszeitraum einer signifikanten Reduktion $(p<0,0001)$, insgesamt gesehen aber einem physiologischen $\mathrm{pH}-$ Wert entspricht. In Tabelle 6.8 (Anhang) sind die Signifikanzniveaus zwischen den verschiedenen Temperaturpunkten zu entnehmen, eine graphische Darstellung findet sich in Abbildung 3.10 und die dazugehörigen Daten in Tabelle 3.14.

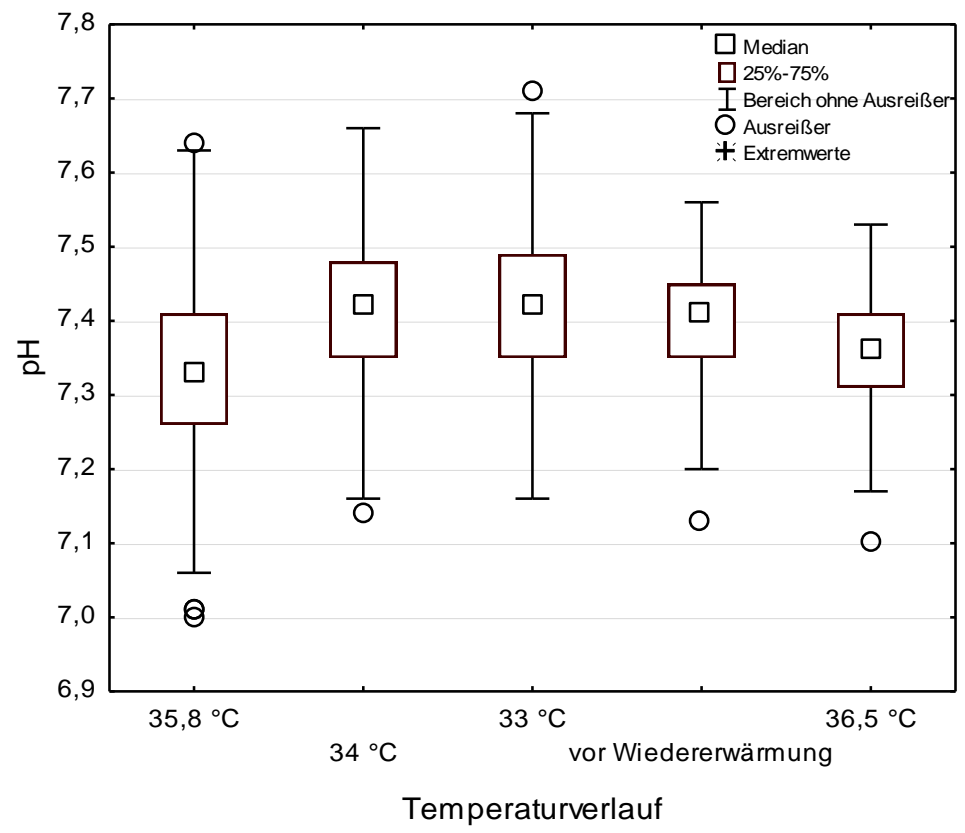

Abb. 3.10: Boxplots zum Verlauf des arteriellen pH-Wertes bei Ankunft auf der Intensivstation $\left(35,8^{\circ} \mathrm{C}\right), 34^{\circ} \mathrm{C}, 33^{\circ} \mathrm{C}$, vor Wiedererwärmung und $36,5^{\circ} \mathrm{C}$ Körperkerntemperatur. $\mathrm{n}=159$.

\begin{tabular}{lrrrrrr}
\hline pH & Median & MW & MIN & MAX & 25\%-Q & $\mathbf{7 5 \% - Q}$ \\
\hline Ankunft ITS & 7,33 & 7,33 & 7,00 & 7,64 & 7,26 & 7,41 \\
$34^{\circ} \mathrm{C}$ & 7,42 & 7,41 & 7,14 & 7,66 & 7,35 & 7,48 \\
$33^{\circ} \mathrm{C}$ & 7,42 & 7,42 & 7,16 & 7,71 & 7,35 & 7,49 \\
vor Wiedererwärmung & 7,41 & 7,40 & 7,13 & 7,56 & 7,35 & 7,45 \\
$36,5^{\circ} \mathrm{C}$ & 7,36 & 7,36 & 7,10 & 7,53 & 7,31 & 7,41 \\
\hline
\end{tabular}

Tab. 3.14: Kennzahlen zum arteriellen pH-Wert innerhalb der einzelnen Temperaturpunkte Ankunft auf der Intensivstation (ITS: $\left.35,8^{\circ} \mathrm{C}\right), 34^{\circ} \mathrm{C}, 33^{\circ} \mathrm{C}$, vor Wiedererwärmung und $36,5^{\circ} \mathrm{C}$ Körperkerntemperatur. $\mathrm{n}=159$.

MW: Mittelwert; MIN: Minimum; MAX: Maximum; 25\%-Q: 25\%-Quantil; 75\%-Q: 75\%-Quantil. 


\section{Diskussion}

Ziel dieser Arbeit war es, zu analysieren, inwiefern eine milde therapeutische Hypothermie die respiratorische und Herz-Kreislauf-Funktion während des Kühlungszeitraums nach erfolgreicher kardiopulmonaler Reanimation beeinflusst.

\subsection{Einfluss milder Hypothermie auf die respiratorische Funktion}

Die MTH wird leitliniengerecht als Standardtherapie nach CPR in der UMG eingesetzt. Eine vielfach angewandte Methode zur Kühlungsinduktion ist dabei die großvolumige Infusion kalter Flüssigkeit. Da Patienten im Rahmen des PCAS häufig eine myokardiale Dysfunktion und eine systemische Entzündungsreaktion (SIRS) aufweisen (Laurent et al., 2002), bestehen Bedenken bezüglich der Induktion eines Lungenödems.

Daher wurden in dieser Arbeit die respiratorischen Parameter von 166 Patienten, die nach erfolgreicher Reanimation mit kaltem Volumen behandelt wurden, analysiert.

Ein Maß zur Beurteilung der Oxygenierungsfunktion der Lunge bzw. der Schwere eines Lungenschadens ist der Horovitz-Quotient $\left(\mathrm{PaO}_{2} / \mathrm{FiO}_{2}\right)$. Seit 2011 existiert gemäß der „Berlin-Definition“ folgende Einteilung des Akuten Atemnotsyndroms (ARDS): a) mildes ARDS, wenn der $\mathrm{PaO}_{2} / \mathrm{FiO}_{2}$-Quotient $\leq 300 \mathrm{mmHg}$ beträgt; b) moderates ARDS, wenn der $\mathrm{PaO}_{2} / \mathrm{FiO}_{2}$-Quotient $\leq 200 \mathrm{mmHg}$ beträgt und $\mathrm{c}$ ) schweres ARDS, wenn der $\mathrm{PaO}_{2} / \mathrm{FiO}_{2}$-Quotient $\leq 100 \mathrm{mmHg}$ beträgt (Ranieri et al., 2012). Die Daten dieser Arbeit zeigen, dass bei Ankunft auf der ITS im Median der Horovitz-Quotient der Patienten bei $210,5 \mathrm{mmHg}$ lag. Dies entspricht einem milden ARDS an der Grenze zum moderaten ARDS. Im Verlauf der Kühlungsphase blieb der $\mathrm{PaO}_{2} / \mathrm{FiO}_{2}$-Quotient annähernd unverändert $\left(34^{\circ} \mathrm{C}: 210,3 \mathrm{mmHg} ; 33^{\circ} \mathrm{C}: 208,0\right.$ $\mathrm{mmHg}$; n.s., $\mathrm{p}=0$,8598). Der PEEP wurde im gleichen Zeitraum sehr leicht, aber signifikant angehoben, im Median verblieb er jedoch zu allen Temperaturpunkten bei 7 mbar. Dabei erhielten die Patienten bis zum Erreichen einer Zieltemperatur von $<34^{\circ} \mathrm{C}$ im Mittel $3.279 \pm 151 \mathrm{ml}$ kalte Infusionen und bis zum Erreichen von $33^{\circ} \mathrm{C}$ im Mittel $4.095 \pm 177 \mathrm{ml}$. Die initiale EF betrug im Mittel 34,8 $\pm 1,1 \%$, was einer mittelgradig eingeschränkten LV-Funktion entspricht (Lang et al., 2005). 
Die Ergebnisse der Regressionsanalyse zwischen der Menge an infundiertem kaltem Volumen bis zu einer KKT von $34^{\circ} \mathrm{C}$ und dem Horovitz-Quotienten zeigten einen leichten, aber signifikanten negativen linearen Zusammenhang zwischen der Menge an infundierter Flüssigkeit und der Lungenfunktion $\left(r^{2}=0,06 ; p=0,0014\right)$. Keine signifikanten Korrelationen bestanden zwischen der Menge an infundierter Flüssigkeit und der Änderungsrate des $\mathrm{PaO}_{2} / \mathrm{FiO}_{2}$-Quotienten sowie der $\mathrm{EF}$ und der Änderungsrate des $\mathrm{PaO}_{2} / \mathrm{FiO}_{2}$-Quotienten.

Bereits eine Pilotstudie an 52 reanimierten Patienten hatte gezeigt, dass in der Postreanimationsphase ein moderates Lungenversagen besteht. Hier zeigte sich keine signifikante Verschlechterung der Lungenfunktion durch eine schnelle Infusion von kaltem Volumen (Jacobshagen et al., 2009).

$\mathrm{Zu}$ ähnlichen Ergebnissen bezüglich der respiratorischen Funktion von Patienten nach CPR kommen auch andere Studien. So konnten Kim et al. in einer prospektiven Fallserie mit 17 OHCA-Patienten zeigen, dass die rasche Infusion 2 । kalten Volumens trotz mittelgradig eingeschränkter LVEF vor Infusionsbeginn (34 $\pm 18,6 \%$ ) nicht zu einer Verschlechterung der Lungenfunktion führt (Kim et al., 2005).

In einer retrospektiven Studie aus dem Jahr 2010, in der 62 Patienten eingeschlossen waren, wurde der Einfluss der MTH auf die Beatmungskonditionen bei OHCA-Patienten untersucht. Die Hypothermie wurde mittels Infusion von RingerLaktat-Lösung $\left(4^{\circ} \mathrm{C} ; 100 \mathrm{ml} / \mathrm{min}\right)$ und Kühlmatratzen induziert. Es konnte gezeigt werden, dass der Horovitz-Quotient während des Herunterkühlens auf die Zieltemperatur bei unverändertem PEEP signifikant anstieg (von 233 auf 268 $\mathrm{mmHg}$ ), während der 24-stündigen Kühlungsphase allerdings wieder signifikant zurückging auf $244 \mathrm{mmHg}$ (Aslami et al., 2010).

Hier ist zu berücksichtigen, dass die Messwerte in dieser Studie nicht temperaturkorrigiert wurden, was insbesondere auch einen Einfluss auf den $\mathrm{PaO}_{2}$ und somit auf den Horovitz-Quotienten hat. Bei einer Temperatur $<37^{\circ} \mathrm{C}$ sind $\mathrm{PaO}_{2}$ und $\mathrm{PaCO}_{2}$ infolge der erhöhten Löslichkeit niedriger, der arterielle $\mathrm{pH}-$ Wert steigt. Eindeutig ist jedoch, dass es zu keiner Verschlechterung der Lungenfunktion kam.

Auf der anderen Seite wurde von Hammer et al. eine signifikant höhere Rate an bilateralen Lungeninfiltrationen nach prähospitaler Volumengabe in der ersten Röntgen-Thorax-Aufnahme nach Ankunft im Krankenhaus dokumentiert, im 
Vergleich zu einer Kontrollgruppe ohne Erhalt einer MTH (41\% zu 16\%; $p=0,02)$. Diese Veränderungen in den Röntgenaufnahmen gingen allerdings nicht mit einer Hypoxämie einher und der Horovitz-Quotient bei Ankunft auf der ITS war bei beiden Gruppen annähernd gleich (246 mmHg zu $225 \mathrm{mmHg}$; $\mathrm{m}=0$,73) (Hammer et al., 2009).

Bernard et al. zeigten 2003 in einer Studie mit 22 eingeschlossenen OHCAPatienten, dass trotz rascher Volumeninfusion bei keinem der Patienten ein Lungenödem nachgewiesen werden konnte. Außerdem führte die Gabe kalten Volumens zu einer schnellen Reduktion der KKT (von 35,5 auf $33,8^{\circ} \mathrm{C}$ nach $30 \mathrm{~min}$ ) sowie zu einer signifikanten Verbesserung des mittleren arteriellen Blutdrucks, der Nierenfunktion und des Säure-Basen-Status (Bernard et al., 2003).

$\mathrm{Zu}$ ähnlichen Ergebnissen kamen auch Kim et al. In einer randomisierten klinischen Studie mit 125 eingeschlossenen Patienten konnten nach präklinischer Infusion von 500-2.000 $\mathrm{ml} 4^{\circ} \mathrm{C}$ kalter Infusionen weder ein Lungenödem im ersten RöntgenThorax noch eine verschlechterte arterielle Oxygenierung nachgewiesen werden (Kim et al., 2007).

Diese Ergebnisse lassen schlussfolgern, dass der Einsatz kalter Infusionen zur Induktion der MTH nicht zu einer relevanten Verschlechterung der Oxygenierungsfunktion der Lunge führt. Eine Erklärung hierfür könnte sein, dass Patienten nach CPR einen erhöhten Volumenbedarf haben. In früheren Studien zeigte sich, dass es im Rahmen des PCAS häufig zu einem SIRS kommt (von manchen Autoren auch „sepsis-like-syndrome“ genannt), welches ursächlich für den vermehrten Volumenbedarf ist (Adrie et al., 2002; Neumar et al., 2008). Aufgrund der systemischen Entzündungsreaktion mit einhergehender Extravasation und Vasodilatation kann es zu einem intravasalen Volumenmangel kommen. Daher benötigen die Patienten eine Volumensubstitution zum Ausgleich der Hypovolämie, so dass die kalten Infusionen nicht zu einer Volumenüberladung führen.

In einer ersten Studie zeigte sich, dass die in der Sepsis-Therapie nach Leitlinien eingesetzte „early goal-directed therapy“ zur hämodynamischen Optimierung der Patienten in Kombination mit MTH eine Verbesserung des Gesamtüberlebens bei OHCA-Patienten bietet. Innerhalb der ersten zwölf Stunden nach ROSC erhielten die Patienten im Mittel 5.761 ml (2.250-14.795 ml) gekühlte Infusionen zur Induktion der MTH und Stabilisierung des Kreislaufs (Gaieski et al., 2009). 
Nordmark et al. zeigten in einer Studie mit 24 eingeschlossenen ROSC-Patienten und anschließender Kühlungsinduktion mittels $4^{\circ} \mathrm{C}$ kalter Infusionen, dass ein Großteil der Patienten trotz positiver Flüssigkeitsbilanz eine Hypovolämie aufwies. Zwölf Stunden nach ROSC wurde per Echo bei zehn bzw. 13 von 23 Patienten ein verringertes intravaskuläres Volumen diagnostiziert, nach 24 bzw. $48 \mathrm{~h}$ stieg diese Zahl noch an (14 bzw. 13/19 und 13 bzw. 12/21 Patienten). Die EF blieb in diesem Zeitraum konstant (Median-LVEF 56\% nach 12 h, 62\% nach 24 h, 59\% nach 48 h) (Nordmark et al., 2009).

Auch Laurent et al. stellten dar, dass bei OHCA-Patienten mit myokardialer Dysfunktion in den ersten $24 \mathrm{~h}$ nach ROSC ein Gesamtvolumen von $5 \mathrm{I}(3,5-6,5 \mathrm{I})$ an (nicht gekühlten) Kristalloiden zur Stabilisierung eines zentralen Venendrucks von $>12 \mathrm{mmHg}$ notwendig waren (Laurent et al., 2002).

In der Zusammenschau der Daten zeigt sich, dass die rasche Infusion kalten Volumens nicht zu einer Verschlechterung der respiratorischen Funktion bei reanimierten Patienten führt. Der zusätzliche Volumenbedarf im Rahmen eines SIRS ist ein wichtiges Argument für die Gabe kalten Volumens zur Induktion einer milden Hypothermie. Da in dieser Arbeit jedoch eine geringe Korrelation zwischen der Menge an infundierter Flüssigkeit und dem Horovitz-Quotienten gezeigt werden konnte, sollten die respiratorischen Parameter bei der Gabe großer Volumina engmaschig überwacht werden.

\subsection{Kühlungsgeschwindigkeit unter Gabe kalter Infusionen}

Nach HKS wird ein schnellstmöglicher Kühlungsbeginn und schnellstmögliches Erreichen der Zieltemperatur von $\leq 34^{\circ} \mathrm{C}$ von dem ERC empfohlen; auch wenn keiner speziellen Kühlungsmethode der Vorrang gegeben wird (Deakin et al., 2010). In dieser Arbeit wurde die Kühlung mittels Infusionen, teilweise in Kombination mit anderen Kühlungsmethoden (CoolGard ${ }^{\circledR}$, Kühlzelt, Eispacks usw.), schnellstmöglich nach Erreichen der UMG eingeleitet und während etwaiger Untersuchungen (Koronarangiographie, CT etc.) fortgeführt. Die Patienten benötigten bis zum Erreichen einer Zieltemperatur von $<34^{\circ} \mathrm{C}$ im Mittel $4,5 \pm 0,3 \mathrm{~h}$ mit einer Kühlungsgeschwindigkeit von $0,62 \pm 0,04^{\circ} \mathrm{C} / \mathrm{h}$. 
Im Vergleich hierzu berichteten Bernard et al. von einer Geschwindigkeit von $3,4^{\circ} \mathrm{C} / \mathrm{h}$ (Bernard et al., 2003), während Hoedemaekers et al. eine Kühlgeschwindigkeit von $0,31 \pm 0,23^{\circ} \mathrm{C} / \mathrm{h}$ dokumentierten. Es gab andere Kühlungsmethoden, die eine schnellere Kühlungsgeschwindigkeit aufwiesen (Kühlungsmatten: $1,33 \pm 0,63^{\circ} \mathrm{C} / \mathrm{h}$; CoolGard $^{\circledR}: 1,46 \pm 0,42^{\circ} \mathrm{C} / \mathrm{h}$ ) (Hoedemaekers et al., 2007). Zu bedenken ist hier jedoch, dass diese Methoden während apparativer Untersuchungen (z.B. CT oder Herzkatheter) hinderlich sein können. Kühlungskatheter sind darüber hinaus invasiv und können zu entsprechenden Komplikationen führen (Blutung, Infektion, Thrombose etc.).

Ursachen für eine geringere Kühlungsgeschwindigkeit durch Infusionen können sein, dass die Infusionen nicht kalt genug sind (Taskinen et al., 2010), die Infusionsgeschwindigkeit nicht hoch genug ist oder die "Kühlkette“ nicht korrekt eingehalten wird (Becker, 2010).

Ein weiteres Problem des Einsatzes kalter Infusionen kann sein, dass die Aufrechterhaltung einer $\mathrm{KKT}$ von $33^{\circ} \mathrm{C}$ nicht stabil gelingt (Kliegel et al., 2007). Schon in Kombination mit Eispacks ist sie jedoch sehr effektiv und ebenfalls kostensparend (Larsson et al., 2010).

In der Zusammenschau lässt sich sagen, dass die Infusion kalten Volumens zu einer raschen Induktion der Hypothermie führt und dabei auch während Untersuchungen einfach durchzuführen ist. Zur Aufrechterhaltung der MTH ist die Kombination mit anderen Verfahren empfehlenswert.

\subsection{Einfluss milder Hypothermie auf die Herz-Kreislauf-Funktion}

Im Rahmen des PCAS weisen Patienten häufig eine myokardiale Dysfunktion mit einhergehender Kreislaufinstabilität auf. Diese spricht gewöhnlich auf eine (inotrope) Katecholamin-Therapie an, wenn eine Volumengabe alleine nicht effektiv ist (Laurent et al., 2002; Neumar et al., 2008). Es gibt allerdings Hinweise, dass die MTH selbst einen positiv inotropen Effekt besitzt (Weisser et al., 2001; Jacobshagen et al., 2010). Ebenfalls findet sich häufig eine beeinträchtigte Vasoregulation nach ROSC, die evtl. einer Vasopressor-Therapie bedarf (Neumar et al., 2008).

In dieser Dissertation wurden die Herz-Kreislauf-Parameter von 159 gekühlten Patienten nach erfolgreicher Reanimation analysiert. Dabei konnte gezeigt werden, 
dass die MTH die hämodynamische Situation der Patienten stabilisiert und zu einer verminderten HF führt.

\subsubsection{Einfluss auf die Adrenalin-Dosierung}

Adrenalin führt über die $\beta_{1}$-adrenerge Wirkung zu einer Erhöhung des HZV (positive Inotropie) sowie über $\alpha_{1}$-adrenerge Wirkung zu einer Engstellung peripherer Gefäße (Burchardi et al., 2011).

In dieser Arbeit zeigte sich, dass die Adrenalin-Dosierung unter MTH signifikant gesenkt werden konnte. Sie betrug bei Ankunft auf der ITS im Mittel 5,9 $\mu \mathrm{g} / \mathrm{min}$, bei $34^{\circ} \mathrm{C} 2,8 \mu \mathrm{g} / \mathrm{min}$ und bei $33^{\circ} \mathrm{C} 1,4 \mu \mathrm{g} / \mathrm{min}$.

Diese Ergebnisse stehen im Einklang mit früheren Studien und unterstreichen die positiv inotrope Wirkung der Hypothermie. Diese konnte bereits in mehreren Studien am Tiermodell gezeigt werden (Shattock and Bers, 1987; Weisser et al., 2001). Und auch in Studien an humanem Myokard (in vitro) konnte sie nachgewiesen werden (Weisser et al., 2001; Jacobshagen et al., 2010). So demonstrierten Weisser et al. 2001 an isolierten Ventrikel-Myokard-Streifen (Mensch und Schwein), dass bei schrittweiser Temperaturänderung von $37^{\circ} \mathrm{C}$ auf $31^{\circ} \mathrm{C}$ die isometrische Kontraktionskraft signifikant zunahm (gesundes humanes Myokard: 91\%; SchweineMyokard: $50 \% ; 31^{\circ} \mathrm{C}$ gegen $\left.37^{\circ} \mathrm{C} ; \mathrm{p}<0.05\right)$. Die Erhöhung der Kontraktionskraft um 90\% in humanem Myokard entspricht dabei dem positiv inotropen Effekt eines mittleren Levels an Katecholamin-Stimulation (Pieske et al., 1997). Auch die Kontraktions- und Relaxationszeit nahm zu. Dabei änderten sich die $\mathrm{Ca}^{2+}$ Konzentrationen im Sarkoplasmatischen Retikulum (SR) und im Zytosol nicht signifikant. In anästhesierten Schweinen wurden zusätzlich die hämodynamischen Parameter unter Hypothermie beobachtet. Die HF sank von 111 auf 73/min, während das HZV von 2,4 auf $3,1 \mathrm{l} / \mathrm{min}$ anstieg $\left(37^{\circ} \mathrm{C}\right.$ gegen $\left.31^{\circ} \mathrm{C} ; \mathrm{p}<0,05\right)$. Systemischer und pulmonaler Gefäßwiderstand veränderten sich nicht signifikant (Weisser et al., 2001). Jacobshagen et al. kamen 2010 zu ähnlichen Ergebnissen. In vitro wurden humane Herzmuskelstreifen terminal herzinsuffizienter Patienten von $37^{\circ} \mathrm{C}$ bis auf $27^{\circ} \mathrm{C}$ gekühlt. Hierunter erhöhte sich die Kontraktionskraft auf im Maximum 167,5\% $(p<0,05)$. Auch die Kontraktions- und Relaxationszeiten waren verlängert. Für insuffizientes Myokard konnte unter Hypothermie zudem eine signifikante Erhöhung 
der $\mathrm{Ca}^{2+}-$ Konzentration im SR gezeigt werden. Aber auch unter vollständiger Blockade des SR erhöhte sich die Kontraktionskraft signifikant auf 145,1\% ( $p<0,05)$. Dies spricht für eine Zunahme der $\mathrm{Ca}^{2+}$-Sensitivität als Mechanismus der positiv inotropen Wirkung der Hypothermie (Jacobshagen et al., 2010). Hierfür ist die Erhöhung der Kontraktions- und Relaxationszeit typisch. Sie zeichnet sich durch eine signifikante Erhöhung der Zeit bis zur Maximalkontraktion, der totalen Kontraktionszeit und der Zeit bis zur 50\%igen bzw. 90\%igen Relaxation aus (Blinks and Endoh, 1986; Weisser et al., 2001; Jacobshagen et al., 2010). Und auch im schweren kardiogenen Schock scheint die MTH eine sichere Methode zu sein, um die Herzfunktion zu verbessern (Schmidt-Schweda et al., 2013).

Auf der anderen Seite fanden Goetzenich et al. einen negativ inotropen Effekt unter Hypothermie (von $37^{\circ} \mathrm{C}$ auf $31^{\circ} \mathrm{C}$ ) auf humanes Atrium-Myokard. Es kam zu einer verminderten Kontraktionskraft und $\mathrm{Ca}^{2+}$-Konzentration im $\mathrm{SR}$ von humanen AtriumMyokard-Zellen im Vergleich zu einer erhöhten Kontraktionskraft sowie $\mathrm{Ca}^{2+}$ Konzentration im SR von Kaninchen-Myokard (Atrium und Ventrikel). Jedoch war die Kontraktions- und Relaxationszeit sowohl in humanem als auch Kaninchen-Myokard ebenfalls verlängert (Goetzenich et al., 2009).

Bernard et al. berichteten von einem generell erhöhten Adrenalin-Bedarf unter Hypothermie (59\% der Patienten benötigten Adrenalin-Unterstützung) gegenüber der Normothermie-Gruppe (49\%). Die kumulative Dosis innerhalb der ersten 24 Stunden war allerdings in beiden Gruppen gleich (2,2 $\pm 2,1 \mathrm{mg}$ gegen 2,2 $\pm 1,9 \mathrm{mg}$ ) (Bernard et al., 2002). Da in dieser Studie nur über zwölf Stunden gekühlt wurde, schloss dieser Kumulativwert somit auch den Wiedererwärmungs-Zeitraum ein. Die Daten sind daher nur bedingt mit den Ergebnissen dieser Dissertation vergleichbar und schließen sich nicht aus.

Im Gegensatz zu Weisser et al. (siehe oben) beschrieben Bernard et al. zudem einen im Vergleich zur Normothermie-Gruppe geringeren $\mathrm{Cl}$ unter MTH bei allerdings gleichzeitig erhöhtem systemischen Gefäßwiderstand als wahrscheinlichste Ursache (Weisser et al., 2001; Bernard et al., 2002).

In der Zusammenschau der Studien kann somit von einer positiv inotropen Wirkung der Hypothermie selbst ausgegangen werden. 
Die MTH könnte auch im Hinblick auf den Myokardinfarkt einen positiven Effekt haben. Im Tier-Modell wurde beobachtet, dass Hypothermie die potenzielle Risikozone nach Myokardinfarkt signifikant verringert (Miki et al., 1998). In der vorliegenden Studie wurde initial eine EF von im Mittel 34,7 $\pm 1,1 \%$ diagnostiziert (121/159 Patienten). Bei einer Verlaufskontrolle (75/159 Patienten) verbesserte sich die EF auf $41,5 \pm 1,5 \%$. Möglicherweise ist diese Verbesserung der EF auf einen protektiven Effekt der Hypothermie zurückzuführen. Es fehlt jedoch eine Kontrollgruppe.

\subsubsection{Einfluss auf die Noradrenalin-Dosierung}

Noradrenalin (NA) führt über eine $\alpha_{1}$-adrenerge Stimulation zu einer Vasokonstriktion und dadurch zu einer Erhöhung des totalen peripheren Widerstandes (TPR) (Burchardi et al., 2011). In dieser Arbeit zeigte sich, dass die NA-Dosierung innerhalb des Kühlungszeitraums signifikant gesteigert werden musste. Patienten die eine $\mathrm{PCl}$ erhielten, wurden dabei im Vergleich zu dem Kollektiv, welches keine PCI benötigte, mit einer signifikant höheren Noradrenalin-Dosierung zu den jeweiligen Temperaturpunkten therapiert.

Die Ergebnisse sprechen dafür, dass nach Reanimation eine vermehrte Vasokonstriktion zur Kreislaufstabilisierung notwendig ist. In einer Studie von Laurent et al. zeigte sich unter Normothermie eine Vasodilatation für bis zu $72 \mathrm{~h}$ nach OHCA trotz optimaler Volumengabe und Normalisierung der myokardialen Dysfunktion (Laurent et al., 2002). Ursächlich ist die im Rahmen des systemischen IschämieReperfusions-Schadens des PCAS entstehende Entzündungsreaktion. Eine daraus resultierende Endothelschädigung führt neben anderen Faktoren zu einer Extravasation und verminderten Vasoregulation im Sinne einer systemischen Vasodilatation mit Hypovolämie und Blutdruckabfall und ist damit neben der myokardialen Dysfunktion eine Ursache für hämodynamische Instabilität nach Reanimation (Böttiger et al., 1995; Adrie et al., 2002; Neumar et al., 2008).

Bernard et al. beschrieben in ihrer Studie aus 2002 eine Assoziation der MTH mit einem erhöhten systemischen Gefäßwiderstand im Vergleich zu der NormothermieKontrollgruppe (Bernard et al., 2002). Diese Wirkung der MTH könnte sich positiv auf die hämodynamische Stabilisierung nach Reanimation auswirken. 
Weisser et al. fanden hingegen keine signifikante Veränderung des systemischen oder pulmonalen Gefäßwiderstandes unter MTH im Tiermodell (Weisser et al., 2001). Zudem kann auch durch den Gebrauch von Sedativa eine weitere Vasodilatation herbeigeführt werden (Nordmark et al., 2009). In dieser Arbeit wurden alle Patienten mittels Midazolam und Fentanyl oder Propofol und Sulfentanyl während des Kühlungszeitraums sediert. Somit könnte dies zumindest ein beeinflussender Faktor für den vermehrten Bedarf an NA unter MTH sein.

Der signifikant höhere NA-Bedarf bei Patienten mit PCI könnte dadurch zu erklären sein, dass Patienten, die wegen eines Myokardinfarktes eine $\mathrm{PCl}$ benötigen, eine schlechtere Myokardfunktion aufweisen, so dass eine erhöhte hämodynamische Instabilität besteht. Laurent et al. beschrieben, dass es bei 54,5\% der Patienten nach OHCA zu einer hämodynamischen Instabilität mit Katecholaminpflichtigkeit kam (Gruppe A). Diese Gruppe hatte während einer Koronarangiographie im Vergleich zur Gruppe ohne hämodynamische Instabilität (Gruppe B) eine signifikant geringere LVEF. Ebenfalls wurde ein signifikant höherer LVEDP in Gruppe A gemessen. Es zeigte sich eine höhere Wahrscheinlichkeit des Eintretens einer hämodynamischen Instabilität, wenn ein akuter Koronarverschluss der Grund des OHCA war (50,7\% gegen 37,3\%; $p=0,06$ ) (Laurent et al., 2002). Aufgrund des verstärkten Schockgeschehens kommt es zu einer gesteigerten systemischen Entzündungsreaktion (SIRS) mit Vasodilatation im Körper, die zu einem erhöhten NA-Bedarf führt.

Zusammenfassend kann gesagt werden, dass es im Rahmen des PCAS zu einer Vasodilatation kommt, die einer Therapie mit Vasopressoren zur Kreislaufstabilisierung bedarf.

\subsubsection{Einfluss auf Blutdruck und Herzfrequenz}

In dieser Dissertation konnte gezeigt werden, dass unter MTH die HF signifikant sinkt. So betrug sie bei der Ankunft auf der ITS im Median 90/min, bei $34^{\circ} \mathrm{C} 70 / \mathrm{min}$ und bei $33^{\circ} \mathrm{C} 65 / \mathrm{min}$. 
Eine moderate Senkung der HF ist vorteilhaft, da bei einem langsamer schlagenden Herzen vor allem die Diastole verlängert ist, so dass die Ventrikel besser mit Blut gefüllt werden können und der Auswurf steigt. Darüber hinaus führt eine verlängerte Diastole zu einer verbesserten Koronardurchblutung (Reil and Böhm, 2007). Zudem wurde an isolierten Kaninchen-Herzen demonstriert, dass eine steigende HF dem positiv inotropen Effekt der MTH entgegenwirken kann. Bei einer niedrigen HF von $<30 / \mathrm{min}$ und gleichzeitiger Kühlung auf $30^{\circ} \mathrm{C}$ stieg der linksventrikuläre endsystolische Druck (LVESP) um 32\% an. Der LVEDP blieb bei dieser HF unabhängig von der Temperatur annähernd gleich niedrig. Bei Erhöhung der HF ging dieser Effekt sukzessive verloren. Bei einer $\mathrm{HF} \geq 90 /$ min stellte sich sogar ein negativ inotoper Effekt ein. So sank der LVESP, während der LVEDP anstieg (Mattheussen et al., 1996). Zu ähnlichen Ergebnissen kommen auch Lewis et al. in einer Studie mit Patienten, die einen koronaren Bypass erhielten. Bei $37^{\circ} \mathrm{C}$ KKT führte eine Erhöhung der HF zu einem Anstieg der Kontraktionskraft. Bei $33^{\circ} \mathrm{C}$ KKT kehrte sich dieser Effekt um (Lewis et al., 2002).

Der MAP betrug in der vorliegenden Arbeit bei der Ankunft auf ITS im Median 83,3 $\mathrm{mmHg}$, bei $34^{\circ} \mathrm{C} 76,7 \mathrm{mmHg}$ sowie $75,0 \mathrm{mmHg}$ bei $33^{\circ} \mathrm{C}$.

Es gibt bisher keine genaue Definition des optimalen MAP nach HKS, da prospektive Studien fehlen. Empfohlen wird von dem ILCOR eine Stabilisierung des MAP, so dass eine adäquate Urinproduktion von $1 \mathrm{ml} / \mathrm{kg} / \mathrm{min}$ erreicht wird. Dies gilt als Indikator für eine ausreichende Organperfusion (Neumar et al., 2008). Wichtig ist, eine ausreichende Perfusion des Gehirns sicherzustellen, was durch einen postischämischen Verlust der cerebrovaskulären Autoregulation erschwert ist. Eine Hypertension sollte dabei ebenfalls vermieden werden. So zeigte sich, dass trotz fünf-minütiger hypertensiver Reperfusion von $>100 \mathrm{mmHg}$ nach ROSC sechs Monate später kein signifikant besseres neurologisches Outcome zu evaluieren war, im Vergleich zu einer Kontrollgruppe mit einem MAP $\leq 100 \mathrm{mmHg}$ (Müllner et al., 1996). Da das Myokard postischämisch ebenfalls stark belastet ist, sollte versucht werden, das Herz so weit wie möglich zu schonen. Der optimale MAP scheint dabei vom jeweiligen Patienten abzuhängen (65-100 mmHg) (Neumar et al., 2008). 
Zusammenfassend gesehen ist eine niedrige HF bei adäquatem MAP nützlich für eine stabile Hämodynamik unter MTH. Die MTH selbst führt dabei zu einer Reduktion der HF.

\subsubsection{Einfluss des pH-Wertes auf die Katecholamin-Dosierung}

Ein wichtiger Einflussfaktor der Katecholamin-Dosierung ist der arterielle $\mathrm{pH}$-Wert. Im Rahmen des PCAS kommt es häufig zu einer metabolischen Azidose. Die Wirksamkeit von Katecholaminen kann dadurch herabgesetzt werden, der Bedarf steigt. Im Umkehrschluss kann es bei einer Stabilisierung des $\mathrm{pH}$ zu einer Verminderung des Katecholamin-Bedarfs kommen. In der vorliegenden Arbeit wurde bei Erreichen der ITS im Median ein arterieller pH-Wert von 7,33 dokumentiert, was einer milden Azidose entspricht. Im Verlauf der Kühlungsperiode normalisierte sich der $\mathrm{pH}$ wieder. Und auch während der 24-stündigen Kühlungsperiode sowie nach Erreichen einer $\mathrm{KKT}$ von $36,5^{\circ} \mathrm{C}$ verblieb der pH im physiologischen Bereich $(7,41$ bzw. 7,36).

Im gleichen Zeitraum konnte die Adrenalin-Dosierung signifikant reduziert werden. Daher könnte es sein, dass die Normalisierung des pH einen Einfluss auf den verminderten Adrenalin-Bedarf ausübte. Allerdings konnte bei einer Änderung der KKT von $34^{\circ} \mathrm{C}$ auf $33^{\circ} \mathrm{C}$ die Adrenalin-Dosierung weiter von im Mittel $2,8 \mu \mathrm{g} / \mathrm{min}$ auf $1,4 \mu \mathrm{g} / \mathrm{min}$ gesenkt werden, obwohl in diesem Zeitraum keine Veränderung des arteriellen $\mathrm{pH}(7,42)$ eintrat.

Daher kann davon ausgegangen werden, dass der arterielle $\mathrm{pH}$ vermutlich keinen Einfluss auf die Verminderung der Adrenalin-Dosierung hatte.

\subsection{Limitationen der Datengewinnung und -interpretation}

Zu berücksichtigen sind Limitationen hinsichtlich der Datenanalyse. Zunächst handelt es sich um zwei retrospektive Studien ohne Kontrollgruppen. So konnten die Daten bezüglich respiratorischer und hämodynamischer Parameter unter MTH nicht mit Normothermie-Gruppen verglichen werden. Da jedoch ab 2004 die MTH in der UMG bei allen Patienten eingesetzt wurde, die nach erfolgreicher Reanimation bewusstlos blieben, wäre die Normothermie-Kontrollgruppe stark selektiert gewesen nach 
Patienten mit kurzem HKS und kurzer Reanimation. Zudem wurde das jeweilige Kollektiv der Patienten sehr eng gefasst, indem nur Patienten berücksichtigt wurden, für die zu jedem definierten Temperaturpunkt alle relevanten Daten vorhanden waren. Ausgeschlossen wurden zudem die Patienten, die schon vor der Wiedererwärmung auf $36,5^{\circ} \mathrm{C}$ verstorben sind.

Respiratorische Daten: Es kann nicht ausgeschlossen werden, dass der diensthabende Arzt auf der Intensivstation angesichts einer respiratorischen Verschlechterung des Patienten die Infusion kalten Volumens verringerte oder beendete und die Weiterkühlung mittels anderer Kühlungsmethoden erfolgte. Dieses Vorgehen hätte einen beeinflussenden Effekt auf die respiratorische Situation der Patienten und würde die Daten evtl. verändern.

Herz-Kreislauf-Daten: Es erfolgte keine routinemäßige Überwachung mittels PAKatheter, sondern nur in Einzelfällen. Somit konnten keine invasiv gemessenen Hämodynamik-Daten ausgewertet werden. Es besteht allerdings auch keine Evidenz dafür, dass der Gebrauch von PA-Kathetern die Prognose nach HKS verbessert (Neumar et al., 2008).

Zudem hat sich gezeigt, dass die myokardiale Dysfunktion ebenso wie die verminderte Vasoregulation im Rahmen des PCAS eine potenzielle Reversibilität aufweisen (Laurent et al., 2002). Somit kann nicht ausgeschlossen werden, dass eine Stabilisierung des Kreislaufs vielleicht auch ohne MTH erfolgt wäre. Diese Theorie wurde allerdings bisher in keiner Studie bestätigt. Vielmehr berichteten Laurent et al. von einer Zunahme des Bedarfs an Katecholaminen unter normothermen Bedingungen in den ersten acht Stunden nach OHCA bzw. zwölf Stunden danach, auch wenn sich der Kreislauf nach 24-72 h unabhängig davon stabilisierte (Laurent et al., 2002). Zudem zeigten mehrere experimentelle Studien eine erhöhte Kontraktionskraft von Herzmuskelzellen unter Hypothermie auf (Weisser et al., 2001; Jacobshagen et al., 2010). 


\subsection{Ausblick}

Trotz dieser positiven Ergebnisse und der Leitlinien-Empfehlung für die MTH gibt es viele offene Fragen. So ist immer noch unklar, welche Zieltemperatur und welche Kühlungsdauer optimal sind. Auch gibt es bislang keine Evidenz dafür, welche Kühlungsmethode für den Patienten am besten ist. Fraglich ist zudem, ob eine Kühlung auch nach intrahospitalem HKS sinnvoll ist. Die optimalen Zielgrößen von Atmungs- und Herz-Kreislauf-Parametern unter MTH sowie die beste Sedierungsform sind ebenfalls noch nicht definiert.

Für diese Fragen sind prospektive Studien notwendig, um zu evaluieren, welche Maßnahmen das Überleben bzw. das neurologische Outcome von Patienten nach Reanimation weiter verbessern. 


\section{Zusammenfassung}

Die Überlebensrate von Reanimations-Patienten ist seit Jahrzehnten gleichbleibend gering. Die milde therapeutische Hypothermie (MTH) hat als bisher einzige Therapieoption gezeigt, dass das Gesamtüberleben und neurologische Outcome nach Wiedererlangen eines Kreislaufs signifikant verbessert wurde. Eine mögliche Kühlungsmethode ist die Hochvolumengabe $2-4^{\circ} \mathrm{C}$ kalter Infusionen zur Induktion und Aufrechterhaltung einer Körperkerntemperatur (KKT) von $<34^{\circ} \mathrm{C}$ über 12-24 Stunden. Da nach kardiopulmonaler Reanimation häufig eine myokardiale Dysfunktion sowie eine systemische Entzündungsreaktion (SIRS) mit einhergehender Kreislaufinstabilität im Rahmen des Postreanimationssyndroms (PCAS) auftreten, könnten Bedenken bezüglich der Induktion eines Lungenödems bestehen. In dieser Arbeit wurde daher der Einfluss von kalten Infusionen auf die respiratorische Funktion untersucht. Ebenfalls wurde evaluiert, inwiefern sich die milde therapeutische Hypothermie auf die Katecholamin-Dosierung zur Kreislaufstabilisierung sowie auf Herz-Kreislauf-Parameter auswirkt. Dazu wurden retrospektiv die Daten von 166 Patienten bezüglich respiratorischer Parameter und von 159 Patienten bezüglich kardialer Parameter während des Kühlungszeitraums statistisch analysiert.

Respiratorische Daten: Die Ejektionsfraktion (EF) betrug bei Aufnahme 34,8\%. Dies entspricht einer mittelgradig eingeschränkten linksventrikulären (LV)-Funktion. Bei Ankunft auf der Intensivstation betrug der Horovitz-Quotient $\left(\mathrm{PaO}_{2} / \mathrm{FiO}_{2}\right)$ als $\mathrm{Maß}$ für die Oxygenierungsfunktion der Lunge $210 \mathrm{mmHg}$ bei einem PEEP von $7 \mathrm{mbar}$. Dies entspricht einem milden bis moderaten akuten Atemnotsyndrom (ARDS). Bis zu einer Zieltemperatur von $\leq 34^{\circ} \mathrm{C}(4,5 \mathrm{~h})$ wurden $3.279 \mathrm{ml}$ kaltes Volumen infundiert. Der Horovitz-Quotient verblieb dennoch unverändert bei $210 \mathrm{mmHg}$, der PEEP bei 7 mbar. Es zeigte sich ein schwacher, aber signifikanter, negativer linearer Zusammenhang zwischen Horovitz-Quotient und der Menge an infundiertem kaltem Volumen bei einer infundierten Flüssigkeitsmenge zwischen 0-11.000 ml.

Diese Ergebnisse zeigen, dass die Oxygenierungsfunktion der Lunge durch die Volumengabe nicht beeinträchtigt wird. Dennoch sollten aufgrund des schwachen linearen Zusammenhangs von Infusionsmenge und Horovitz-Quotient die 
respiratorischen Parameter während der Gabe großer Mengen an kaltem Volumen engmaschig überwacht werden.

Herz-Kreislauf-Daten: Initial bestand eine mittelgradig eingeschränkte LV-Funktion (EF 34,7\%), die sich im Verlauf auf 40,9\% verbesserte. Unter MTH konnte die Infusionsrate von Adrenalin signifikant reduziert werden, die Noradrenalin-Dosierung wurde hingegen signifikant erhöht. Die Herzfrequenz sank im gleichen Zeitraum signifikant.

Diese Ergebnisse bestätigen vorherige Studien, die zeigen, dass die MTH eine positiv inotrope Wirkung besitzt und die HF senkt. Der zusätzliche Bedarf an Vasokonstriktoren ist wahrscheinlich auf eine im Rahmen des PCAS auftretende Vasodilatation zurückzuführen. 


\section{Anhang}

\section{1 statistische Daten}

\begin{tabular}{lll}
\hline Parameter & Test & p-Wert \\
\hline $\mathrm{PaO} 2$ & Haupteffekt & $<0,0001$ \\
& ITS gegen $34^{\circ} \mathrm{C}$ & $=0,001$ \\
& ITS gegen $33^{\circ} \mathrm{C}$ & $<0,0001$ \\
& $34^{\circ} \mathrm{C}$ gegen $33^{\circ} \mathrm{C}$ & $=0,1830$ \\
$\mathrm{FiO2}$ & Haupteffekt & $<0,0001$ \\
& ITS gegen $34^{\circ} \mathrm{C}$ & $<0,0001$ \\
& ITS gegen $33^{\circ} \mathrm{C}$ & $<0,0001$ \\
& $34^{\circ} \mathrm{C}$ gegen $33^{\circ} \mathrm{C}$ & $=0,0004$ \\
$\mathrm{PEEP}$ & Haupteffekt & $=0,002$ \\
& ITS gegen $34^{\circ} \mathrm{C}$ & $=0,0012$ \\
& ITS gegen $33^{\circ} \mathrm{C}$ & $=0,0180$ \\
& $34^{\circ} \mathrm{C}$ gegen $33^{\circ} \mathrm{C}$ & $=0,9946$ \\
\hline $\mathrm{PaO} 2 / \mathrm{FiO} 2$ & Haupteffekt & $=0,8598$ \\
& ITS gegen $34^{\circ} \mathrm{C}$ & - \\
\hline ITS gegen $33^{\circ} \mathrm{C}$ & - \\
\hline $34^{\circ} \mathrm{C}$ gegen $33^{\circ} \mathrm{C}$ & - \\
\hline
\end{tabular}

Tab. 6.1: Nichtparametrische ANOVA mit Paarvergleichen zu den Parametern arterieller SauerstoffPartialdruck $\left(\mathrm{PaO}_{2}\right)$, inspiratorische Sauerstoffkonzentration $\left(\mathrm{FiO}_{2}\right)$, positiver endexspiratorischer Druck (PEEP) und Horovitz-Quotient $\left(\mathrm{PaO}_{2} / \mathrm{FiO}_{2}\right) . \mathrm{n}=166$.

\begin{tabular}{lll}
\hline Parameter & Faktor & p-Wert \\
\hline Noradrenalin & Temperatur & $<0,0001$ \\
& $\mathrm{PCl}$ & $=0,0890$ \\
& Wechselwirkung & $=0,0031$ \\
Adrenalin & Temperatur & $<0,0001$ \\
& $\mathrm{PCl}$ & $=0,1524$ \\
& Wechselwirkung & $=0,4061$ \\
Dobutamin & Temperatur & $=0,2655$ \\
& PCl & $=0,0418$ \\
& Wechselwirkung & $=0,3312$ \\
MAP & Temperatur & $<0,0001$ \\
& PCl & $=0,1812$ \\
& Wechselwirkung & $=0,9715$ \\
HF & Temperatur & $<0,0001$ \\
& PCl & $=0,1219$ \\
& Wechselwirkung & $=0,0815$ \\
& Temperatur & $<0,0001$ \\
& PCl & $=0,0244$ \\
& Wechselwirkung & $=0,3669$ \\
\hline
\end{tabular}

Tab. 6.2: Nichtparametrische ANOVA zu den Parametern Noradrenalin, Adrenalin, Dobutamin, mittlerem arteriellen Blutdruck (MAP), Herzfrequenz (HF) und arteriellem pH-Wert. PCl: perkutane koronare Intervention. $\mathbf{n}=166$. 


\begin{tabular}{lllll}
\hline Noradrenalin & $\mathbf{3 4}^{\circ} \mathbf{C}$ & $\mathbf{3 3}^{\circ} \mathbf{C}$ & vor Wiedererwärmung & $\mathbf{3 6 , 5 ^ { \circ } \mathbf { C }}$ \\
\hline Ankunft ITS & $=0,0237$ & $=0,0132$ & $<0,0001$ & $<0,0001$ \\
$34^{\circ} \mathrm{C}$ & - & n.s. & $=0,0223$ & $<0,0001$ \\
$33^{\circ} \mathrm{C}$ & & - & $=0,0175$ & $<0,0001$ \\
vor Wiedererwärmung & & & - & $=0,0166$ \\
$36,5^{\circ} \mathrm{C}$ & & & & - \\
\hline
\end{tabular}

Tab. 6.3: Paarvergleiche der Noradrenalin-Dosierung zwischen den einzelnen Temperaturpunkten Ankunft Intensivstation (ITS: $35,8^{\circ} \mathrm{C}$ ), $34^{\circ} \mathrm{C}, 33^{\circ} \mathrm{C}$, vor Wiedererwärmung und $36,5^{\circ} \mathrm{C}$ Körperkerntemperatur des Subkollektivs $\mathrm{PCI}=\mathbf{0}$ (keine perkutane koronare Intervention erhalten). $\mathbf{n}=95$. n.s.: nicht signifikant.

\begin{tabular}{lllll}
\hline Noradrenalin & $\mathbf{3 4}^{\circ} \mathbf{C}$ & $\mathbf{3 3}^{\circ} \mathbf{C}$ & vor Wiedererwärmung & $\mathbf{3 6 , 5 ^ { \circ } \mathbf { C }}$ \\
\hline Ankunft ITS & $<0,0001$ & $<0,0001$ & $<0,0001$ & $<0,0001$ \\
$34^{\circ} \mathrm{C}$ & - & n.s. & $=0,0119$ & n.s. \\
$33^{\circ} \mathrm{C}$ & & - & n.s. & n.s. \\
vor Wiedererwärmung & & & - & n.s. \\
$36,5^{\circ} \mathrm{C}$ & & & & - \\
\hline
\end{tabular}

Tab. 6.4: Paarvergleiche der Noradrenalin-Dosierung zwischen den einzelnen Temperaturpunkten Ankunft auf der Intensivstation (ITS: $\left.35,8^{\circ} \mathrm{C}\right), 34^{\circ} \mathrm{C}, 33^{\circ} \mathrm{C}$, vor Wiedererwärmung und $36,5^{\circ} \mathrm{C}$ Körperkerntemperatur des Subkollektivs $\mathbf{P C l}=\mathbf{1}$ (perkutane koronare Intervention erhalten). $\mathbf{n}=$ 64. n.s.: nicht signifikant.

\begin{tabular}{lllll}
\hline Adrenalin & $\mathbf{3 4}^{\circ} \mathbf{C}$ & $\mathbf{3 3}^{\circ} \mathbf{C}$ & vor Wiedererwärmung & $\mathbf{3 6 , 5 ^ { \circ } \mathbf { C }}$ \\
\hline Ankunft ITS & $<0,0001$ & $<0,0001$ & $<0,0001$ & $<0,0001$ \\
$34^{\circ} \mathrm{C}$ & - & n.s. & n.s. & n.s. \\
$33^{\circ} \mathrm{C}$ & & - & n.s. & n.s. \\
vor Wiedererwärmung & & & - & n.s. \\
$36,5^{\circ} \mathrm{C}$ & & & & - \\
\hline
\end{tabular}

Tab. 6.5: Paarvergleiche der Adrenalin-Dosierung zwischen den einzelnen Temperaturpunkten Ankunft auf der Intensivstation (ITS: $35,8^{\circ} \mathrm{C}$ ), $34^{\circ} \mathrm{C}, 33^{\circ} \mathrm{C}$, vor Wiedererwärmung und $36,5^{\circ} \mathrm{C}$ Körperkerntemperatur. $n=159$. n.s.: nicht signifikant.

\begin{tabular}{lllll}
\hline MAP & $\mathbf{3 4}^{\circ} \mathbf{C}$ & $\mathbf{3 3}^{\circ} \mathbf{C}$ & vor Wiedererwärmung & $\mathbf{3 6 , 5 ^ { \circ } \mathbf { C }}$ \\
\hline Ankunft ITS & $=0,0203$ & $=0,0007$ & $<0,0001$ & $<0,0001$ \\
$34^{\circ} \mathrm{C}$ & - & n.s. & $=0,0003$ & $=0,007$ \\
$33^{\circ} \mathrm{C}$ & & - & $=0,0235$ & n.s. \\
vor Wiedererwärmung & & & - & n.s. \\
$36,5^{\circ} \mathrm{C}$ & & & & - \\
\hline
\end{tabular}

Tab. 6.6: Paarvergleiche des mittleren arterielle Blutdrucks (MAP) zwischen den einzelnen Temperaturpunkten Ankunft auf der Intensivstation (ITS: $35,8^{\circ} \mathrm{C}$ ), $34^{\circ} \mathrm{C}, 33^{\circ} \mathrm{C}$, vor Wiedererwärmung und $36,5^{\circ} \mathrm{C}$ Körperkerntemperatur. $\mathrm{n}=159$. n.s.: nicht signifikant. 


\begin{tabular}{lllll}
\hline HF & $\mathbf{3 4}^{\circ} \mathbf{C}$ & $\mathbf{3 3}^{\circ} \mathbf{C}$ & vor Wiedererwärmung & $\mathbf{3 6 , 5}^{\circ} \mathbf{C}$ \\
\hline Ankunft ITS & $<0,0001$ & $<0,0001$ & $<0,0001$ & n.s. \\
$34^{\circ} \mathrm{C}$ & - & $<0,0001$ & $=0,0028$ & $<0,0001$ \\
$33^{\circ} \mathrm{C}$ & - & n.s. & $<0,0001$ \\
vor Wiedererwärmung & & - & $<0,0001$ \\
$36,5^{\circ} \mathrm{C}$ & & & - \\
\hline
\end{tabular}

Tab. 6.7: Paarvergleiche der Herzfrequenz (HF) zwischen den einzelnen Temperaturpunkten Ankunft auf der Intensivstation (ITS: $35,8^{\circ} \mathrm{C}$ ), $34^{\circ} \mathrm{C}, 33^{\circ} \mathrm{C}$, vor Wiedererwärmung und $36,5^{\circ} \mathrm{C}$ Körperkerntemperatur. $\mathrm{n}=159$. n.s.: nicht signifikant.

\begin{tabular}{lllll}
\hline pH-Wert & $\mathbf{3 4}^{\circ} \mathbf{C}$ & $\mathbf{3 3}^{\circ} \mathbf{C}$ & vor Wiedererwärmung & $\mathbf{3 6 , 5 ^ { \circ } \mathbf { C }}$ \\
\hline Ankunft ITS & $<0,0001$ & $<0,0001$ & $<0,0001$ & n.s. \\
$34^{\circ} \mathrm{C}$ & - & n.s. & n.s. & $<0,0001$ \\
$33^{\circ} \mathrm{C}$ & & - & n.s. & $<0,0001$ \\
vor Wiedererwärmung & & & - & $<0,0001$ \\
$36,5^{\circ} \mathrm{C}$ & & & & - \\
\hline
\end{tabular}

Tab. 6.8: Paarvergleiche des arteriellen pH-Wertes zwischen den einzelnen Temperaturpunkten Ankunft auf der Intensivstation (ITS: $35,8^{\circ} \mathrm{C}$ ), $34^{\circ} \mathrm{C}, 33^{\circ} \mathrm{C}$, vor Wiedererwärmung und $36,5^{\circ} \mathrm{C}$ Körperkerntemperatur. $n=159$. n.s.: nicht signifikant. 


\subsection{Abbildungen und Daten nach Aufteilung $\mathrm{PCI}=0$ und $\mathrm{PCI}=1$}

\subsubsection{Adrenalin-Dosierung}

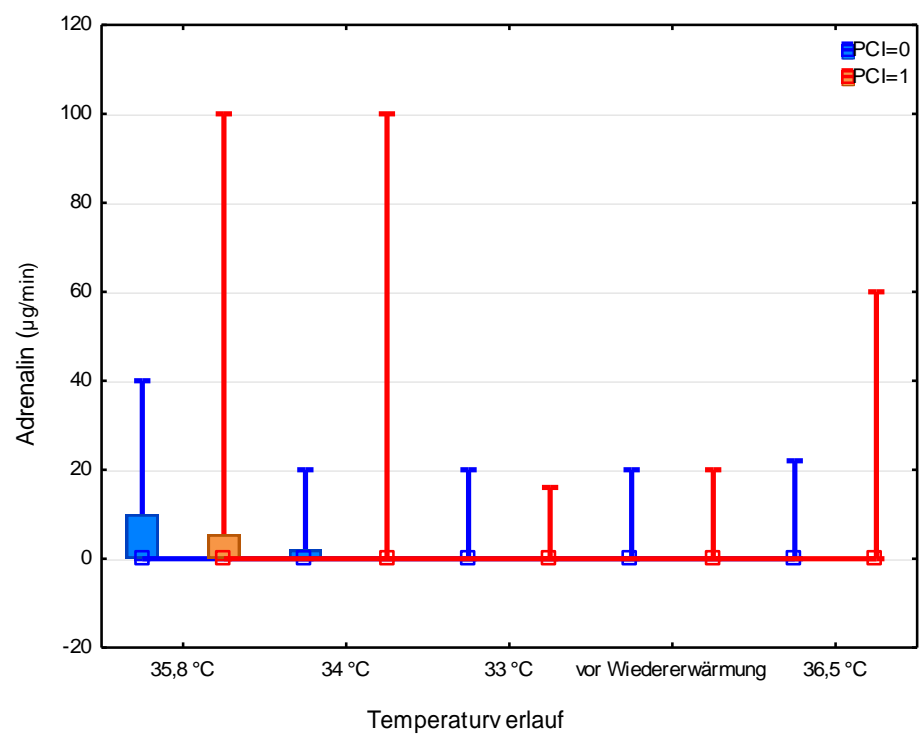

Abb. 6.1: Boxplots zum Verlauf der Adrenalin-Dosierung bei Ankunft auf Intensivstation $\left(35,8^{\circ} \mathrm{C}\right)$, bei $34^{\circ} \mathrm{C}, 33^{\circ} \mathrm{C}$, vor Wiedererwärmung und $36,5^{\circ} \mathrm{C}$ Körperkerntemperatur. Aufspaltung in $\mathbf{P C l}=\mathbf{0}$ (keine perkutane koronare Intervention erhalten; $n=95)$ und $\mathrm{PCl}=1(\mathrm{PCl}$ erhalten; $n=64)$.

\begin{tabular}{lrrrrrrr}
\hline Adrenalin $(\mu \mathrm{g} / \mathrm{min})$ & Median & MW & MIN & MAX & $\mathbf{2 5 \% - Q}$ & $\mathbf{7 5 \% - Q}$ & Ant. \\
\hline Ankunft ITS & 0,0 & 5,5 & 0,0 & 40,0 & 0,0 & 10,0 & $39 / 95$ \\
$34^{\circ} \mathrm{C}$ & 0,0 & 2,5 & 0,0 & 20,0 & 0,0 & 2,0 & $28 / 95$ \\
$33^{\circ} \mathrm{C}$ & 0,0 & 1,7 & 0,0 & 20,0 & 0,0 & 0,0 & $23 / 95$ \\
vor Wiedererwärmung & 0,0 & 1,9 & 0,0 & 20,0 & 0,0 & 0,0 & $22 / 95$ \\
$36,5^{\circ} \mathrm{C}$ & 0,0 & 1,6 & 0,0 & 22,0 & 0,0 & 0,0 & $18 / 95$ \\
\hline
\end{tabular}

Tab. 6.9: Kennzahlen zur Adrenalin-Dosierung innerhalb der einzelnen Temperaturpunkte Ankunft auf der Intensivstation (ITS: $35,8^{\circ} \mathrm{C}$ ), $34^{\circ} \mathrm{C}, 33^{\circ} \mathrm{C}$, vor Wiedererwärmung und $36,5^{\circ} \mathrm{C}$ Körperkerntemperatur des Subkollektivs $\mathbf{P C l}=\mathbf{0}$ (keine perkutane koronare Intervention erhalten). $\mathbf{n}=95$.

MW: Mittelwert; MIN: Minimum; MAX: Maximum; 25\%-Q: 25\%-Quantil; 75\%-Q: 75\%-Quantil; Ant.: Anteil der mit Adrenalin behandelten Patienten von der Gesamtzahl der Patienten.

\begin{tabular}{lrrrrrrr}
\hline Adrenalin $(\boldsymbol{\mu g} / \mathbf{m i n})$ & Median & MW & MIN & MAX & $\mathbf{2 5 \% - Q}$ & $\mathbf{7 5 \% - Q}$ & Ant. \\
\hline Ankunft ITS & 0,0 & 6,4 & 0,0 & 100,0 & 0,0 & 5,5 & $25 / 64$ \\
$34^{\circ} \mathrm{C}$ & 0,0 & 3,2 & 0,0 & 100,0 & 0,0 & 0,0 & $13 / 64$ \\
$33^{\circ} \mathrm{C}$ & 0,0 & 0,8 & 0,0 & 16,0 & 0,0 & 0,0 & $10 / 64$ \\
vor Wiedererwärmung & 0,0 & 0,8 & 0,0 & 20,0 & 0,0 & 0,0 & $7 / 64$ \\
$36,5^{\circ} \mathrm{C}$ & 0,0 & 1,9 & 0,0 & 60,0 & 0,0 & 0,0 & $9 / 64$ \\
\hline
\end{tabular}

Tab. 6.10: Kennzahlen zur Adrenalin-Dosierung innerhalb der einzelnen Temperaturpunkte Ankunft auf der Intensivstation (ITS: $\left.35,8^{\circ} \mathrm{C}\right), 34^{\circ} \mathrm{C}, 33^{\circ} \mathrm{C}$, vor Wiedererwärmung und $36,5^{\circ} \mathrm{C}$ Körperkerntemperatur des Subkollektivs $\mathbf{P C I}=1$ (perkutane koronare Intervention erhalten). $\mathbf{n}=$ 64.

MW: Mittelwert; MIN: Minimum; MAX: Maximum; 25\%-Q: 25\%-Quantil; 75\%-Q: 75\%-Quantil; Ant.: Anteil der mit Adrenalin behandelten Patienten von der Gesamtzahl der Patienten. 


\subsubsection{Dobutamin-Dosierung}

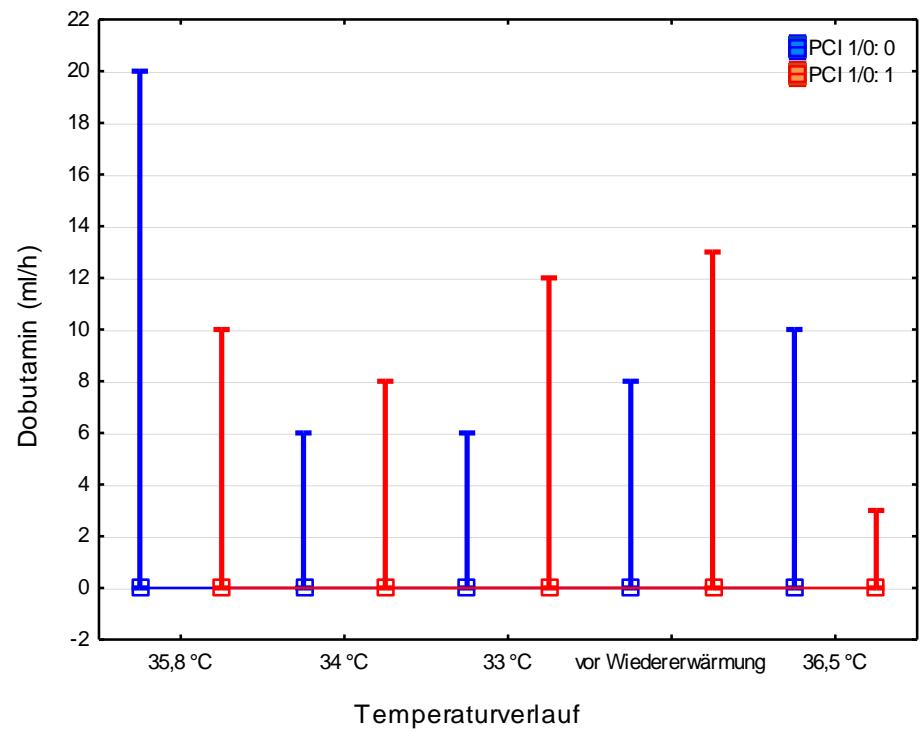

Abb. 6.2: Boxplots zum Verlauf der Dobutamin-Dosierung bei Ankunft auf der Intensivstation $\left(35,8^{\circ} \mathrm{C}\right)$, bei $34^{\circ} \mathrm{C}, 33^{\circ} \mathrm{C}$, vor Wiedererwärmung und $36,5^{\circ} \mathrm{C}$ Körperkerntemperatur. Aufspaltung in $\mathrm{PCl}=0$ (keine perkutane koronare Intervention erhalten; $n=95)$ und $\mathrm{PCl}=1(\mathrm{PCl}$ erhalten; $n=$ 64).

\begin{tabular}{lrrrrrrr}
\hline Dobutamin $(\mathbf{m I} / \mathbf{h})$ & Median & MW & MIN & MAX & $\mathbf{2 5 \% - Q}$ & $\mathbf{7 5 \% - Q}$ & Ant. \\
\hline Ankunft ITS & 0,0 & 0,3 & 0,0 & 20,0 & 0,0 & 0,0 & $4 / 95$ \\
$34^{\circ} \mathrm{C}$ & 0,0 & 0,1 & 0,0 & 6,0 & 0,0 & 0,0 & $4 / 95$ \\
$33^{\circ} \mathrm{C}$ & 0,0 & 0,2 & 0,0 & 6,0 & 0,0 & 0,0 & $6 / 95$ \\
vor Wiedererwärmung & 0,0 & 0,5 & 0,0 & 8,0 & 0,0 & 0,0 & $10 / 95$ \\
$36,5^{\circ} \mathrm{C}$ & 0,0 & 0,4 & 0,0 & 10,0 & 0,0 & 0,0 & $9 / 95$ \\
\hline
\end{tabular}

Tab. 6.11: Kennzahlen zur Dobutamin-Dosierung innerhalb der einzelnen Temperaturpunkte Ankunft auf der Intensivstation (ITS: $\left.35,8^{\circ} \mathrm{C}\right), 34^{\circ} \mathrm{C}, 33^{\circ} \mathrm{C}$, vor Wiedererwärmung und $36,5^{\circ} \mathrm{C}$ Körperkerntemperatur des Subkollektivs $\mathrm{PCl}=\mathbf{0}$ (keine perkutane koronare Intervention erhalten). $\mathbf{n}=95$.

MW: Mittelwert; MIN: Minimum; MAX: Maximum; 25\%-Q: 25\%-Quantil; 75\%-Q: 75\%-Quantil; Ant.: Anteil der mit Dobutamin behandelten Patienten von der Gesamtzahl der Patienten.

\begin{tabular}{lrrrrrrr}
\hline Dobutamin $(\mathbf{m I} / \mathbf{h})$ & Median & MW & MIN & MAX & $\mathbf{2 5 \% - Q}$ & $\mathbf{7 5 \% - Q}$ & Ant. \\
\hline Ankunft ITS & 0,0 & 0,6 & 0,0 & 10,0 & 0,0 & 0,0 & $6 / 64$ \\
$34^{\circ} \mathrm{C}$ & 0,0 & 0,6 & 0,0 & 8,0 & 0,0 & 0,0 & $10 / 64$ \\
$33^{\circ} \mathrm{C}$ & 0,0 & 0,9 & 0,0 & 12,0 & 0,0 & 0,0 & $11 / 64$ \\
vor Wiedererwärmung & 0,0 & 0,7 & 0,0 & 13,0 & 0,0 & 0,0 & $10 / 64$ \\
$36,5^{\circ} \mathrm{C}$ & 0,0 & 0,2 & 0,0 & 3,0 & 0,0 & 0,0 & $5 / 64$ \\
\hline
\end{tabular}

Tab. 6.12: Kennzahlen zur Dobutamin-Dosierung innerhalb der einzelnen Temperaturpunkte Ankunft auf der Intensivstation (ITS: $\left.35,8^{\circ} \mathrm{C}\right), 34^{\circ} \mathrm{C}, 33^{\circ} \mathrm{C}$, vor Wiedererwärmung und $36,5^{\circ} \mathrm{C}$ Körperkerntemperatur des Subkollektivs $\mathbf{P C I}=\mathbf{1}$ (perkutane koronare Intervention erhalten). $\mathbf{n}=$ 64.

MW: Mittelwert; MIN: Minimum; MAX: Maximum; 25\%-Q: 25\%-Quantil; 75\%-Q: 75\%-Quantil; Ant.: Anteil der mit Dobutamin behandelten Patienten von der Gesamtzahl der Patienten. 


\subsubsection{Mittlerer arterieller Blutdruck}

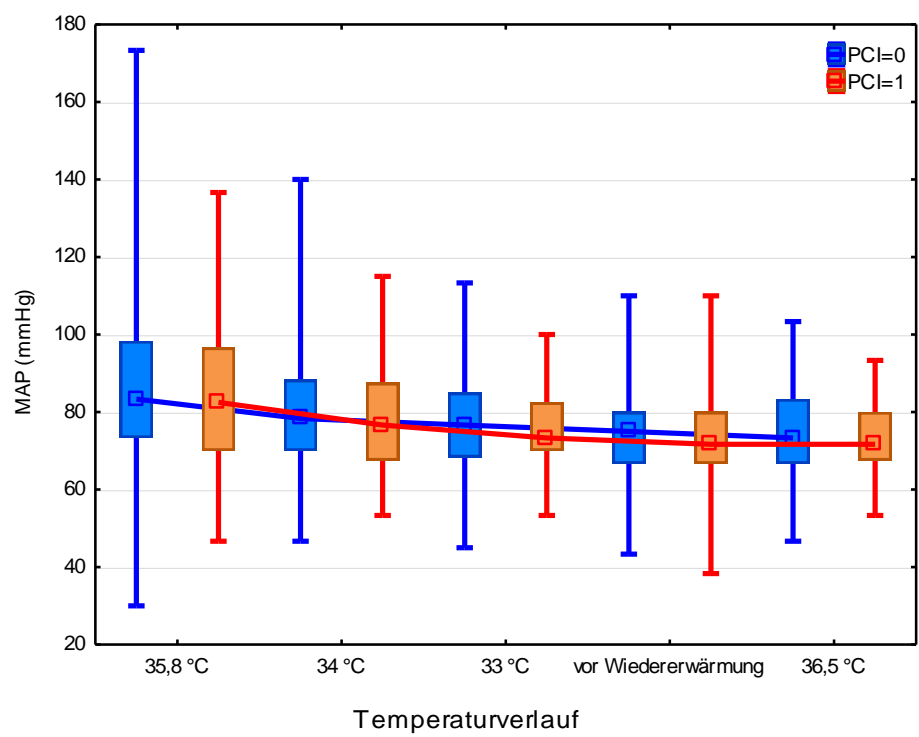

Abb. 6.3: Boxplots zum Verlauf des mittleren arteriellen Blutdruckes (MAP) bei Ankunft auf der Intensivstation $\left(35,8^{\circ} \mathrm{C}\right)$, bei $34^{\circ} \mathrm{C}, 33^{\circ} \mathrm{C}$, vor Wiedererwärmung und $36,5^{\circ} \mathrm{C}$ Körperkerntemperatur. Aufspaltung in $\mathrm{PCl}=0$ (keine perkutane koronare Intervention erhalten; $\mathrm{n}=95$ ) und $\mathrm{PCl}=1$ (PCl erhalten; $n=64)$.

\begin{tabular}{lrrrrrr}
\hline MAP (mmHg) & Median & MW & MIN & MAX & 25\%-Q & $\mathbf{7 5 \% - Q}$ \\
\hline Ankunft ITS & 83,3 & 86,4 & 30,0 & 173,3 & 73,3 & 98,3 \\
$34^{\circ} \mathrm{C}$ & 78,3 & 80,5 & 46,7 & 140,0 & 70,0 & 88,3 \\
$33^{\circ} \mathrm{C}$ & 76,7 & 77,4 & 45,0 & 113,3 & 68,3 & 85,0 \\
vor Wiedererwärmung & 75,0 & 74,4 & 43,3 & 110,0 & 66,7 & 80,0 \\
$36,5^{\circ} \mathrm{C}$ & 73,3 & 74,6 & 46,7 & 103,3 & 66,7 & 83,3 \\
\hline
\end{tabular}

Tab. 6.13: Kennzahlen zum mittleren arteriellen Blutdruck (MAP) innerhalb der einzelnen Temperaturpunkte Ankunft auf der Intensivstation (ITS: $\left.35,8^{\circ} \mathrm{C}\right), 34^{\circ} \mathrm{C}, 33^{\circ} \mathrm{C}$, vor Wiedererwärmung und $36,5^{\circ} \mathrm{C}$ Körperkerntemperatur des Subkollektivs $\mathbf{P C l}=\mathbf{0}$ (keine perkutane koronare Intervention erhalten). $\mathbf{n}=\mathbf{9 5}$.

MW: Mittelwert; MIN: Minimum; MAX: Maximum; 25\%-Q: 25\%-Quantil; 75\%-Q: 75\%-Quantil.

\begin{tabular}{lrrrrrr}
\hline MAP (mmHg) & Median & MW & MIN & MAX & 25\%-Q & $\mathbf{7 5 \% - Q ~}$ \\
\hline Ankunft ITS & 82,5 & 84,5 & 46,7 & 136,7 & 70,0 & 96,7 \\
$34^{\circ} \mathrm{C}$ & 76,7 & 77,7 & 53,3 & 115,0 & 67,5 & 87,5 \\
$33^{\circ} \mathrm{C}$ & 73,3 & 76,4 & 53,3 & 100,0 & 70,0 & 82,5 \\
vor Wiedererwärmung & 71,7 & 72,8 & 38,3 & 110,0 & 66,7 & 80,0 \\
$36,5^{\circ} \mathrm{C}$ & 71,7 & 73,4 & 53,3 & 93,3 & 67,5 & 80,0 \\
\hline
\end{tabular}

Tab. 6.14: Kennzahlen des mittleren arteriellen Blutdrucks (MAP) innerhalb der einzelnen Temperaturpunkte Ankunft auf der Intensivstation (ITS: $\left.35,8^{\circ} \mathrm{C}\right), 34^{\circ} \mathrm{C}, 33^{\circ} \mathrm{C}$, vor Wiedererwärmung und $36,5^{\circ} \mathrm{C}$ Körperkerntemperatur des Subkollektivs $\mathbf{P C l}=\mathbf{1}$ (perkutane koronare Intervention erhalten). $\mathbf{n}=64$.

MW: Mittelwert; MIN: Minimum; MAX: Maximum; 25\%-Q: 25\%-Quantil; 75\%-Q: 75\%-Quantil. 


\subsubsection{Herzfrequenz}

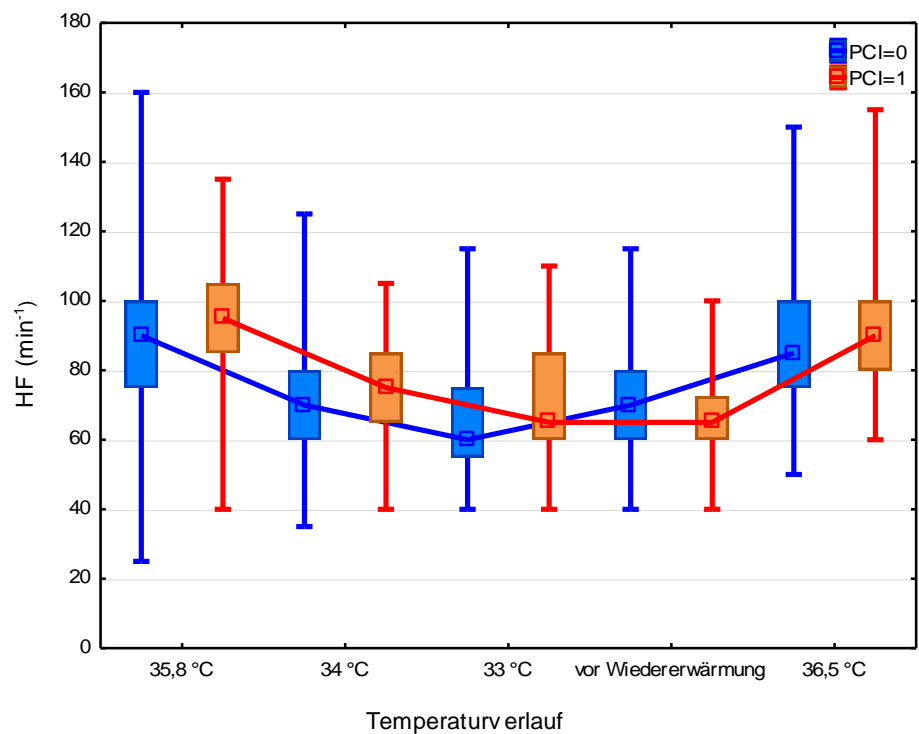

Abb. 6.4: Boxplots zum Verlauf der Herzfrequenz (HF) bei Ankunft auf der Intensivstation $\left(35,8^{\circ} \mathrm{C}\right)$, bei $34^{\circ} \mathrm{C}, 33^{\circ} \mathrm{C}$, vor Wiedererwärmung und $36,5^{\circ} \mathrm{C}$ Körperkerntemperatur. Aufspaltung in $\mathbf{P C l}=\mathbf{0}$ (keine perkutane koronare Intervention erhalten; $n=95)$ und $\mathrm{PCl}=1(\mathrm{PCl}$ erhalten; $n=64)$.

\begin{tabular}{lrrrrrr}
\hline HF $\left(\mathbf{m i n}^{-1}\right)$ & Median & MW & MIN & MAX & 25\%-Q & $\mathbf{7 5 \% - Q}$ \\
\hline Ankunft ITS & 90,0 & 88,6 & 25,0 & 160,0 & 75,0 & 100,0 \\
$34^{\circ} \mathrm{C}$ & 70,0 & 71,7 & 35,0 & 125,0 & 60,0 & 80,0 \\
$33^{\circ} \mathrm{C}$ & 60,0 & 65,6 & 40,0 & 115,0 & 55,0 & 75,0 \\
vor Wiedererwärmung & 70,0 & 68,6 & 40,0 & 115,0 & 60,0 & 80,0 \\
$36,5^{\circ} \mathrm{C}$ & 85,0 & 87,9 & 50,0 & 150,0 & 75,0 & 100,0 \\
\hline
\end{tabular}

Tab. 6.15: Kennzahlen zur Herzfrequenz (HF) innerhalb der einzelnen Temperaturpunkte Ankunft auf der Intensivstation (ITS: $\left.35,8^{\circ} \mathrm{C}\right), 34^{\circ} \mathrm{C}, 33^{\circ} \mathrm{C}$, vor Wiedererwärmung und $36,5^{\circ} \mathrm{C}$ Körperkerntemperatur des Subkollektivs $\mathbf{P C l}=\mathbf{0}$ (keine perkutane koronare Intervention erhalten). $\mathbf{n}=95$.

MW: Mittelwert; MIN: Minimum; MAX: Maximum; 25\%-Q: 25\%-Quantil; 75\%-Q: 75\%-Quantil.

\begin{tabular}{lccccrr}
\hline HF $\left(\mathbf{m i n}^{-1}\right)$ & Median & MW & MIN & MAX & 25\%-Q & $\mathbf{7 5 \% - Q}$ \\
\hline Ankunft ITS & 95,0 & 92,9 & 40,0 & 135,0 & 85,0 & 105,0 \\
$34^{\circ} \mathrm{C}$ & 75,0 & 74,5 & 40,0 & 105,0 & 65,0 & 85,0 \\
$33^{\circ} \mathrm{C}$ & 65,0 & 70,7 & 40,0 & 110,0 & 60,0 & 85,0 \\
vor Wiedererwärmung & 65,0 & 66,6 & 40,0 & 100,0 & 60,0 & 72,5 \\
$36,5^{\circ} \mathrm{C}$ & 90,0 & 90,6 & 60,0 & 155,0 & 80,0 & 100,0 \\
\hline
\end{tabular}

Tab. 6.16: Kennzahlen der Herzfrequenz (HF) innerhalb der einzelnen Temperaturpunkte Ankunft auf der Intensivstation (ITS: $\left.35,8^{\circ} \mathrm{C}\right), 34^{\circ} \mathrm{C}, 33^{\circ} \mathrm{C}$, vor Wiedererwärmung und $36,5^{\circ} \mathrm{C}$ Körperkerntemperatur des Subkollektivs $\mathbf{P C I}=\mathbf{1}$ (perkutane koronare Intervention erhalten). $\mathbf{n}=$ 64.

MW: Mittelwert; MIN: Minimum; MAX: Maximum; 25\%-Q: 25\%-Quantil; 75\%-Q: 75\%-Quantil. 


\subsection{5 pH-Wert}

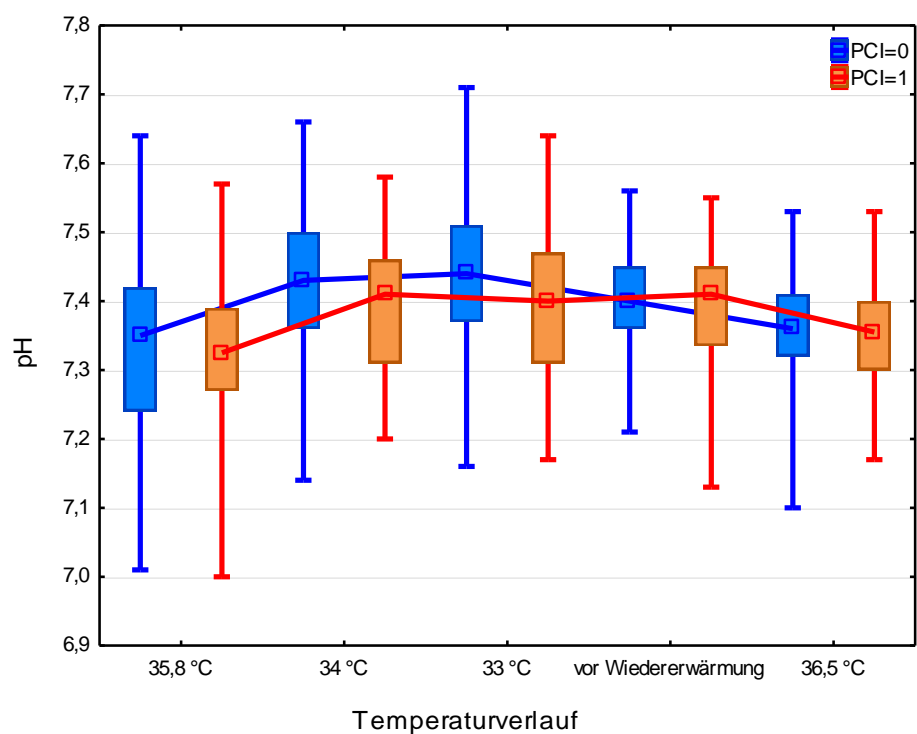

Abb. 6.5: Boxplots zum Verlauf des arteriellen pH-Wertes bei Ankunft auf der Intensivstation $\left(35,8^{\circ} \mathrm{C}\right)$, bei $34^{\circ} \mathrm{C}, 33^{\circ} \mathrm{C}$, vor Wiedererwärmung und $36,5^{\circ} \mathrm{C}$ Körperkerntemperatur. Aufspaltung in $\mathrm{PCl}=0$ (keine perkutane koronare Intervention erhalten; $n=95)$ und $\mathrm{PCl}=1(\mathrm{PCl}$ erhalten; $\mathrm{n}=$ 64).

\begin{tabular}{lrrrrrr}
\hline pH-Wert & Median & MW & MIN & MAX & 25\%-Q & $\mathbf{7 5 \% - Q}$ \\
\hline Ankunft ITS & 7,35 & 7,33 & 7,01 & 7,64 & 7,24 & 7,42 \\
$34^{\circ} \mathrm{C}$ & 7,43 & 7,42 & 7,14 & 7,66 & 7,36 & 7,50 \\
$33^{\circ} \mathrm{C}$ & 7,44 & 7,44 & 7,16 & 7,71 & 7,37 & 7,51 \\
vor Wiedererwärmung & 7,40 & 7,41 & 7,21 & 7,56 & 7,36 & 7,45 \\
$36,5^{\circ} \mathrm{C}$ & 7,36 & 7,36 & 7,10 & 7,53 & 7,32 & 7,41 \\
\hline
\end{tabular}

Tab. 6.17: Kennzahlen zum arteriellen pH-Wert innerhalb der einzelnen Temperaturpunkte Ankunft auf der Intensivstation (ITS: $35,8^{\circ} \mathrm{C}$ ), $34^{\circ} \mathrm{C}, 33^{\circ} \mathrm{C}$, vor Wiedererwärmung und $36,5^{\circ} \mathrm{C}$ Körperkerntemperatur des Subkollektivs $\mathbf{P C I}=\mathbf{0}$ (keine perkutane koronare Intervention erhalten). $\mathbf{n}=\mathbf{9 5}$.

MW: Mittelwert; MIN: Minimum; MAX: Maximum; 25\%-Q: 25\%-Quantil; 75\%-Q: 75\%-Quantil.

\begin{tabular}{lrrrrrr}
\hline pH-Wert & Median & MW & MIN & MAX & 25\%-Q & $\mathbf{7 5 \% - Q}$ \\
\hline Ankunft ITS & 7,33 & 7,33 & 7,00 & 7,57 & 7,27 & 7,39 \\
$34^{\circ} \mathrm{C}$ & 7,41 & 7,39 & 7,20 & 7,58 & 7,31 & 7,46 \\
$33^{\circ} \mathrm{C}$ & 7,40 & 7,39 & 7,17 & 7,64 & 7,31 & 7,47 \\
vor Wiedererwärmung & 7,41 & 7,39 & 7,13 & 7,55 & 7,34 & 7,45 \\
$36,5^{\circ} \mathrm{C}$ & 7,36 & 7,36 & 7,17 & 7,53 & 7,30 & 7,40 \\
\hline
\end{tabular}

Tab.6.18: Kennzahlen zum arteriellen pH-Wert innerhalb der einzelnen Temperaturpunkte Ankunft auf der Intensivstation (ITS: $\left.35,8^{\circ} \mathrm{C}\right), 34^{\circ} \mathrm{C}, 33^{\circ} \mathrm{C}$, vor Wiedererwärmung und $36,5^{\circ} \mathrm{C}$ Körperkerntemperatur des Subkollektivs $\mathbf{P C I}=\mathbf{1}$ (perkutane koronare Intervention erhalten). $\mathbf{n}=$ 64.

MW: Mittelwert; MIN: Minimum; MAX: Maximum; 25\%-Q: 25\%-Quantil; 75\%-Q: 75\%-Quantil. 


\section{Literaturverzeichnis}

Abella BS, Rhee JW, Huang K, Vanden Hoek TL, Becker LB (2005): Induced hypothermia is underused after resuscitation from cardiac arrest: a current practice survey. Resuscitation 64: 181186.

Adrie C, Adib-Conquy M, Laurent I, Monchi M, Vinsonneau C, Fitting C, Fraisse F, Dinh-Xuan AT, Carli P, Spaulding C, et al. (2002): Successful cardiopulmonary resuscitation after cardiac arrest as a "sepsis-like" syndrome. Circulation 106: 562-568.

Arnold U, Pschyrembel W. Pschyrembel klinisches Wörterbuch 2013, 264. Auflage. Gruyter, Berlin 2012.

Aslami H, Binnekade JM, Horn J, Huissoon S, Juffermans NP (2010): The effect of induced hypothermia on respiratory parameters in mechanically ventilated patients. Resuscitation 81: 17231725.

Atwood C, Eisenberg MS, Herlitz J, Rea TD (2005): Incidence of EMS-treated out-of-hospital cardiac arrest in Europe. Resuscitation 67: 75-80.

Balan IS, Fiskum G, Hazelton J, Cotto-Cumba C, Rosenthal RE (2006): Oximetry-guided reoxygenation improves neurological outcome after experimental cardiac arrest. Stroke 37: 30083013.

Becker LB (2010): Cooling heads and hearts versus cooling our heels. Circulation 122: 679-681.

Bernard S, Gray TW, Buist MD, Jones BM, Silvester W, Gutteridge G, Smith K (2002): Treatment of comatose survivors of out-of-hospital cardiac arrest with induced hypothermia. N Engl J Med $\underline{346}$ : $557-563$

Bernard S, Buist M, Monteiro O, Smith K (2003): Induced hypothermia using large volume, ice-cold intravenous fluid in comatose survivors of out-of-hospital cardiac arrest: a preliminary report. Resuscitation 56: 9-13.

Blinks JR, Endoh M (1986): Modification of myofibrillar responsiveness to Ca++ as an inotropic mechanism. Circulation 73: III85-98.

Böttiger BW, Motsch J, Böhrer H, Böker T, Aulmann M, Nawroth PP, Martin E (1995): Activation of blood coagulation after cardiac arrest is not balanced adequately by activation of endogenous fibrinolysis. Circulation 92: 2572-2578.

Böttiger BW, Grabner C, Bauer H, Bode C, Weber T, Motsch J, Martin E (1999): Long term outcome after out-of-hospital cardiac arrest with physician staffed emergency medical services: the Utstein style applied to a midsized urban/suburban area. Heart 82: 674-679.

Braunwald E, Kloner RA (1982): The stunned myocardium: prolonged, postischemic ventricular dysfunction. Circulation 66: 1146-1149.

Brüx A, Girbes ARJ, Polderman KH (2005): Kontrollierte milde und moderate Hypothermie. Anaesthesist 54: 225-244.

Burchardi H, Larsen R, Marx G, Muhl E, Schölmerich J: Die Intensivmedizin, 11. Auflage. Springer Medizin, Berlin 2011.

Buunk G, van der Hoeven JG, Meinders AE (1997): Cerebrovascular reactivity in comatose patients resuscitated from a cardiac arrest. Stroke 28: 1569-1573. 
Churcott CS, Moyes CD, Bressler BH, Baldwin KM, Tibbits GF (1994): Temperature and pH effects on $\mathrm{Ca} 2+$ sensitivity of cardiac myofibrils: a comparison of trout with mammals. Am J Physiol 267: R62-70.

Curry DL, Curry KP (1970): Hypothermia and insulin secretion. Endocrinology 87: 750-755.

Deakin CD, Nolan JP, Soar J, Sunde K, Koster RW, Smith GB, Perkins GD (2010): European Resuscitation Council Guidelines for Resuscitation 2010 Section 4. Adult advanced life support. Resuscitation 81: 1305-1352.

Ehlenbach WJ, Barnato AE, Curtis JR, Kreuter W, Koepsell TD, Deyo RA, Stapleton RD (2009): Epidemiologic study of in-hospital cardiopulmonary resuscitation in the elderly. N Engl J Med 361: 2231.

Europäische Union (2010): Größe und Bevölkerung. http://europa.eu/about-eu/factsfigures/living/index_de.htm.

Fischer M, Hossmann KA (1995): No-reflow after cardiac arrest. Intensive Care Med 21: 132-141.

Gaieski DF, Band RA, Abella BS, Neumar RW, Fuchs BD, Kolansky DM, Merchant RM, Carr BG, Becker LB, Maguire C, et al. (2009): Early goal-directed hemodynamic optimization combined with therapeutic hypothermia in comatose survivors of out-of-hospital cardiac arrest. Resuscitation 80: 418-424.

Gando S, Nanzaki S, Morimoto Y, Kobayashi S, Kemmotsu O (2000): Out-of-hospital cardiac arrest increases soluble vascular endothelial adhesion molecules and neutrophil elastase associated with endothelial injury. Intensive Care Med 26 : 38-44.

Goetzenich A, Schroth SC, Emmig U, Autschbach R, Pieske B, Rossaint R, Christiansen S (2009): Hypothermia exerts negative inotropy in human atrial preparations: in vitro-comparison to rabbit myocardium. J Cardiovasc Surg (Torino) 50: 239-245.

Hachimi-Idrissi S, van Hemelrijck A, Michotte A, Smolders I, Sarre S, Ebinger G, Huyghens L, Michotte Y (2004): Postischemic mild hypothermia reduces neurotransmitter release and astroglial cell proliferation during reperfusion after asphyxial cardiac arrest in rats. Brain Res 1019: 217-225.

Hammer L, Vitrat F, Savary D, Debaty G, Santre C, Durand M, Dessertaine G, Timsit J (2009): Immediate prehospital hypothermia protocol in comatose survivors of out-of-hospital cardiac arrest. Am J Emerg Med 27: 570-573.

Heyndrickx GR, Millard RW, McRitchie RJ, Maroko PR, Vatner SF (1975): Regional myocardial functional and electrophysiological alterations after brief coronary artery occlusion in conscious dogs. J Clin Invest 56: 978-985.

Hoedemaekers CW, Ezzahti M, Gerritsen A, van der Hoeven JG (2007): Comparison of cooling methods to induce and maintain normo- and hypothermia in intensive care unit patients: a prospective intervention study. Crit Care 11: R91.

Holzer M (2010): Targeted temperature management for comatose survivors of cardiac arrest. $N$ Engl J Med 363: 1256-1264.

International Liaison Committee on Resuscitation (2005): Proceedings of the 2005 International Consensus on Cardiopulmonary Resuscitation and Emergency Cardiovascular Care Science with Treatment Recommendations. Resuscitation 67: 157-341.

Jacobshagen C, Pax A, Unsöld BW, Seidler T, Schmidt-Schweda S, Hasenfuss G, Maier LS (2009): Effects of large volume, ice-cold intravenous fluid infusion on respiratory function in cardiac arrest survivors. Resuscitation 80: 1223-1228.

Jacobshagen C, Pelster T, Pax A, Horn W, Schmidt-Schweda S, Unsöld BW, Seidler T, Wagner S, Hasenfuss G, Maier LS (2010): Effects of mild hypothermia on hemodynamics in cardiac arrest survivors and isolated failing human myocardium. Clin Res Cardiol 99: 267-276. 
Kim F, Olsufka M, Carlbom D, Deem S, Longstreth WT, Hanrahan M, Maynard C, Copass MK, Cobb LA (2005): Pilot study of rapid infusion of $2 \mathrm{~L}$ of 4 degrees $C$ normal saline for induction of mild hypothermia in hospitalized, comatose survivors of out-of-hospital cardiac arrest. Circulation 112: 715-719.

Kim F, Olsufka M, Longstreth WT, Maynard C, Carlbom D, Deem S, Kudenchuk P, Copass MK, Cobb LA (2007): Pilot randomized clinical trial of prehospital induction of mild hypothermia in out-ofhospital cardiac arrest patients with a rapid infusion of 4 degrees $C$ normal saline. Circulation 115: 3064-3070.

Kliegel A, Janata A, Wandaller C, Uray T, Spiel A, Losert H, Kliegel M, Holzer M, Haugk M, Sterz F, et al. (2007): Cold infusions alone are effective for induction of therapeutic hypothermia but do not keep patients cool after cardiac arrest. Resuscitation $\underline{73}$ : 46-53.

Klinke, R; Pape, H; Kurtz, A; Silbernagl, S; Baumann, R; Brenner, B; Gay, R; Rothenburger, A: Physiologie, 6. Auflage. Thieme, Stuttgart 2010.

Lang RM, Bierig M, Devereux RB, Flachskampf FA, Foster E, Pellikka PA, Picard MH, Roman MJ, Seward J, Shanewise JS, et al. (2005): Recommendations for chamber quantification: a report from the American Society of Echocardiography's Guidelines and Standards Committee and the Chamber Quantification Writing Group, developed in conjunction with the European Association of Echocardiography, a branch of the European Society of Cardiology. J Am Soc Echocardiogr 18: $1440-1463$.

Langendorff O (1897): Untersuchungen am überlebenden Säugetierherzen. Über den Einfluss von Wärme und Kälte auf das Herz der warmblütigen Tiere. Pflügers Archiv: 355-400.

Larsson I, Wallin E, Rubertsson S (2010): Cold saline infusion and ice packs alone are effective in

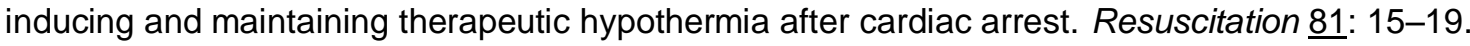

Laurent I, Monchi M, Chiche J, Joly L, Spaulding C, Bourgeois B, Cariou A, Rozenberg A, Carli P, Weber S, et al. (2002): Reversible myocardial dysfunction in survivors of out-of-hospital cardiac arrest. J Am Coll Cardiol $\underline{40}$ : 2110-2116.

Laver S, Farrow C, Turner D, Nolan J (2004): Mode of death after admission to an intensive care unit following cardiac arrest. Intensive Care Med $\underline{30}$ : 2126-2128.

Lewis ME, Al-Khalidi A, Townend JN, Coote J, Bonser RS (2002): The effects of hypothermia on human left ventricular contractile function during cardiac surgery. J Am Coll Cardiol 39: 102-108.

Mattheussen M, Mubagwa K, van Aken H, Wusten R, Boutros A, Flameng W (1996): Interaction of heart rate and hypothermia on global myocardial contraction of the isolated rabbit heart. Anesth Analg 82: 975-981.

Merchant RM, Soar J, Skrifvars MB, Silfvast T, Edelson DP, Ahmad F, Huang K, Khan M, Vanden Hoek TL, Becker LB, et al. (2006): Therapeutic hypothermia utilization among physicians after resuscitation from cardiac arrest. Crit Care Med 34: 1935-1940.

Meybohm P, Gruenewald M, Zacharowski KD, Albrecht M, Lucius R, Fösel N, Hensler J, Zitta K, Bein B (2010): Mild hypothermia alone or in combination with anesthetic post-conditioning reduces expression of inflammatory cytokines in the cerebral cortex of pigs after cardiopulmonary resuscitation. Crit Care 14: R21.

Miki T, Liu GS, Cohen MV, Downey JM (1998): Mild hypothermia reduces infarct size in the beating rabbit heart: a practical intervention for acute myocardial infarction? Basic Res Cardiol 93: 372-383.

Müllner M, Sterz F, Binder M, Hellwagner K, Meron G, Herkner H, Laggner AN (1996): Arterial blood pressure after human cardiac arrest and neurological recovery. Stroke 27: 59-62.

Nadkarni VM, Larkin GL, Peberdy MA, Carey SM, Kaye W, Mancini ME, Nichol G, Lane-Truitt T, Potts J, Ornato JP, et al. (2006): First documented rhythm and clinical outcome from in-hospital cardiac arrest among children and adults. JAMA 295: 50-57. 
Naess A, Steen PA (2004): Long term survival and costs per life year gained after out-of-hospital cardiac arrest. Resuscitation 60: 57-64.

Negovsky VA (1988): Postresuscitation disease. Crit Care Med 16: 942-946.

Neumar RW, Nolan JP, Adrie C, Aibiki M, Berg RA, Böttiger BW, Callaway C, Clark RSB, Geocadin RG, Jauch EC, et al. (2008): Post-cardiac arrest syndrome: epidemiology, pathophysiology, treatment, and prognostication. A consensus statement from the International Liaison Committee on Resuscitation (American Heart Association, Australian and New Zealand Council on Resuscitation, European Resuscitation Council, Heart and Stroke Foundation of Canada, InterAmerican Heart Foundation, Resuscitation Council of Asia, and the Resuscitation Council of Southern Africa); the American Heart Association Emergency Cardiovascular Care Committee; the Council on Cardiovascular Surgery and Anesthesia; the Council on Cardiopulmonary, Perioperative, and Critical Care; the Council on Clinical Cardiology; and the Stroke Council. Circulation 118: 24522483.

Nielsen N, Hovdenes J, Nilsson F, Rubertsson S, Stammet P, Sunde K, Valsson F, Wanscher M, Friberg H (2009): Outcome, timing and adverse events in therapeutic hypothermia after out-ofhospital cardiac arrest. Acta Anaesthesiol Scand 53: 926-934.

Niemann JT, Garner D, Lewis RJ (2004): Tumor necrosis factor-alpha is associated with early postresuscitation myocardial dysfunction. Crit Care Med 32: 1753-1758.

Nolan JP, Morley PT, Hoek TLV, Hickey RW (2003): Therapeutic hypothermia after cardiac arrest. An advisory statement by the Advancement Life support Task Force of the International Liaison committee on Resuscitation. Resuscitation 57: 231-235.

Nolan JP, Deakin CD, Soar J, Böttiger BW, Smith G (2005): European Resuscitation Council guidelines for resuscitation 2005. Section 4. Adult advanced life support. Resuscitation $\underline{67}$ Suppl 1: S39-86.

Nordmark J, Johansson J, Sandberg D, Granstam S, Huzevka T, Covaciu L, Mörtberg E, Rubertsson S (2009): Assessment of intravascular volume by transthoracic echocardiography during therapeutic hypothermia and rewarming in cardiac arrest survivors. Resuscitation 80: 1234-1239.

Nunnally ME, Jaeschke R, Bellingan GJ, Lacroix J, Mourvillier B, Rodriguez-Vega GM, Rubertsson S, Vassilakopoulos T, Weinert C, Zanotti-Cavazzoni S, et al. (2011): Targeted temperature management in critical care: a report and recommendations from five professional societies. Crit Care Med 39: 1113-1125.

Peberdy MA, Kaye W, Ornato JP, Larkin GL, Nadkarni V, Mancini ME, Berg RA, Nichol G, LaneTrultt T (2003): Cardiopulmonary resuscitation of adults in the hospital: a report of 14720 cardiac arrests from the National Registry of Cardiopulmonary Resuscitation. Resuscitation 58: 297-308.

Pell JP, Sirel JM, Marsden AK, Ford I, Walker NL, Cobbe SM (2003): Presentation, management, and outcome of out of hospital cardiopulmonary arrest: comparison by underlying aetiology. Heart $\underline{89}$ : 839-842.

Pieske B, Schlotthauer K, Schattmann J, Beyersdorf F, Martin J, Just H, Hasenfuss G (1997): $\mathrm{Ca}(2+)$-dependent and $\mathrm{Ca}(2+)$-independent regulation of contractility in isolated human myocardium. Basic Res Cardiol 92 Suppl 1: 75-86.

Popp E, Sterz F, Böttiger BW (2005): Therapeutische milde Hypothermie nach Herz-KreislaufStillstand. Anaesthesist 54: 96-106.

Ranieri VM, Rubenfeld GD, Thompson BT, Ferguson ND, Caldwell E, Fan E, Camporota L, Slutsky AS (2012): Acute respiratory distress syndrome: the Berlin Definition. JAMA 307: 2526-2533.

Reil J, Böhm M (2007): The role of heart rate in the development of cardiovascular disease. Clin Res Cardiol 96: 585-592. 
Rivers E, Nguyen B, Havstad S, Ressler J, Muzzin A, Knoblich B, Peterson E, Tomlanovich M (2001): Early goal-directed therapy in the treatment of severe sepsis and septic shock. N Engl J Med 345: 1368-1377.

Rosomoff HL, Holaday DA (1954): Cerebral blood flow and cerebral oxygen consumption during hypothermia. Am J Physiol 179: 85-88.

Ruiz-Bailén M, Aguayo de Hoyos E, Ruiz-Navarro S, Díaz-Castellanos MA, Rucabado-Aguilar L, Gómez-Jiménez FJ, Martínez-Escobar S, Moreno RM, Fierro-Rosón J (2005): Reversible myocardial dysfunction after cardiopulmonary resuscitation. Resuscitation $\underline{66}$ : 175-181.

Sasson C, Rogers MAM, Dahl J, Kellermann AL (2010): Predictors of survival from out-of-hospital cardiac arrest: a systematic review and meta-analysis. Circ Cardiovasc Qual Outcomes 3: 63-81.

Schmidt-Schweda S, Ohler A, Post H, Pieske B (2013): Moderate hypothermia for severe cardiogenic shock (COOL Shock Study I \& II). Resuscitation 84: 319-325.

Schneider A, Albertsmeier M, Böttiger BW, Teschendorf P (2012): Postreanimationssyndrom. Rolle der Entzündung nach Herz-Kreislauf-Stillstand. Anaesthesist 61: 424-436.

Schwertz H, Müller-Werdan U, Prondzinsky R, Werdan K, Buerke M (2004): Katecholamine im kardiogenen Schock: hilfreich, nutzlos oder gefährlich? Dtsch Med Wochenschr 129: 1925-1930.

Shattock MJ, Bers DM (1987): Inotropic response to hypothermia and the temperature-dependence of ryanodine action in isolated rabbit and rat ventricular muscle: implications for excitation-contraction coupling. Circ Res 61: 761-771.

Statistisches Bundesamt (2010): Sterbefälle insgesamt 2010 nach den 10 häufigsten Todesursachen der International Statistical Classification of Diseases and Related Health Problems (ICD-10).

https://www.destatis.de/DE/ZahlenFakten/GesellschaftStaat/Gesundheit/Todesursachen/Tabellen/Ster befaellelnsgesamt.html;jsessionid=24EC585A94B06C53639A5E31AB57292E.cae1.

Stephenson HE, Reid LC, Hinton JW (1953): Some common denominators in 1200 cases of cardiac arrest. Ann Surg 137: 731-744.

Szydlowska K, Tymianski M (2010): Calcium, ischemia and excitotoxicity. Cell Calcium 47: 122-129.

Tamargo J, Duarte J, Caballero R, Delpón E (2011): New therapeutic targets for the development of positive inotropic agents. Discov Med 12: 381-392.

Taskinen T, Puolakka J, Kuisma M (2010): How cool are your "ice-cold" infusions? Resuscitation 81: 921-922.

The Hypothermia after Cardiac Arrest Study Group (2002): Mild therapeutic hypothermia to improve the neurologic outcome after cardiac arrest. N Engl J Med 346: 549-556.

Voicu S, Sideris G, Deye N, Dillinger J, Logeart D, Broche C, Vivien B, Brun P, Capan DD, Manzo-Silberman S, et al. (2012): Role of cardiac troponin in the diagnosis of acute myocardial infarction in comatose patients resuscitated from out-of-hospital cardiac arrest. Resuscitation 83: 452458.

Vreede-Swagemakers JJ de, Gorgels AP, Dubois-Arbouw WI, van Ree JW, Daemen MJ, Houben LG, Wellens HJ (1997): Out-of-hospital cardiac arrest in the 1990's: a population-based study in the Maastricht area on incidence, characteristics and survival. J Am Coll Cardiol 30: 1500-1505.

Weisser J, Martin J, Bisping E, Maier LS, Beyersdorf F, Hasenfuss G, Pieske B (2001): Influence of mild hypothermia on myocardial contractility and circulatory function. Basic Res Cardiol 96: 198205. 
Wolfrum S, Radke PW, Pischon T, Willich SN, Schunkert H, Kurowski V (2007): Mild therapeutic hypothermia after cardiac arrest - a nationwide survey on the implementation of the ILCOR guidelines in German intensive care units. Resuscitation 72: 207-213.

Xu L, Yenari MA, Steinberg GK, Giffard RG (2002): Mild hypothermia reduces apoptosis of mouse neurons in vitro early in the cascade. J Cereb Blood Flow Metab 22: 21-28. 